\title{
Zero dimensional Donaldson-Thomas invariants of threefolds
}

\author{
JUN LI
}

Using a homotopy approach, we prove in this paper a conjecture of Maulik, Nekrasov, Okounkov and Pandharipande on the dimension zero Donaldson-Thomas invariants of all smooth complex threefolds.

$14 \mathrm{D} 20 ; 14 \mathrm{~J} 60$

\section{Introduction}

Ever since the pioneer work of Donaldson and Thomas on Yang-Mills theory over Calabi-Yau threefolds [5; 13], people have been searching for their roles in the study of Calabi-Yau geometry and their relations with other branches of mathematics. The recent results and conjectures of Maulik, Nekrasov, Okounkov and Pandharipande [10; 11] that relate the invariants of the moduli of ideal sheaves of curves on a Calabi-Yau manifold to its Gromov-Witten invariants constitute major progress in this direction. This paper will address one of their conjectures on invariants associated to Hilbert scheme of points.

To begin with, we let $(X, H)$ be a smooth projective threefold over complex numbers $\mathbb{C}$. For any integer $r \geq 0$, a line bundle $I \in \operatorname{Pic}(X)$ and two classes $c_{2} \in H^{4}(X, \mathbb{Z})$ and $c_{3} \in H^{6}(X, \mathbb{Z})$, we form the moduli space

$$
\mathfrak{M}_{X}^{H}\left(r, I, c_{2}, c_{3}\right)
$$

of $H$-stable sheaves of $\mathcal{O}_{X}$-modules $\mathcal{E}$ satisfying

$$
\operatorname{rk} \mathcal{E}=r, \quad \operatorname{det} \mathcal{E}=I, \quad c_{2}(\mathcal{E})=c_{2} \quad \text { and } \quad c_{3}(\mathcal{E})=c_{3} .
$$

Back in the seventies, Maruyama [9] proved that such moduli spaces are quasi-projective, and become projective in case the quaduple $\left(r, I, c_{2}, c_{3}\right)$ is relatively prime. Later, Mukai [12] showed that the first order deformations of any sheaf $\mathcal{E}$ in these moduli spaces are given by the traceless part of the extension group $\operatorname{Ext}^{1}(\mathcal{E}, \mathcal{E})$; the obstructions 
to deforming $\mathcal{E}$ lie in the traceless part of $\operatorname{Ext}^{2}(\mathcal{E}, \mathcal{E})$ (see also Artamkin [1]). In case $X$ is a Calabi-Yau threefold and $\mathcal{E}$ is stable, the traceless part

$$
\operatorname{Ext}^{3}(\mathcal{E}, \mathcal{E})_{0}=\operatorname{Ext}^{0}(\mathcal{E}, \mathcal{E})_{0}^{\vee}=0
$$

while

$$
\operatorname{Ext}^{2}(\mathcal{E}, \mathcal{E})_{0}=\operatorname{Ext}^{1}(\mathcal{E}, \mathcal{E})_{0}^{\vee}
$$

Hence the moduli space $\mathfrak{M}_{X}^{H}\left(r, I, c_{2}, c_{3}\right)$ admits a perfect-obstruction theory as defined by Li and Tian [7] and Behrend and Fantechi [4]; when it is projective, it carries a virtual dimension zero cycle

$$
\left[\mathfrak{M}_{X}^{H}\left(r, I, c_{2}, c_{3}\right)\right]^{\mathrm{vir}} \in H_{0}\left(\mathfrak{M}_{X}^{H}\left(r, I, c_{2}, c_{3}\right), \mathbb{Z}\right) .
$$

Its degree is the invariant originally defined and studied by Donaldson and Thomas [5]. Following [10; 11], we shall call them Donaldson-Thomas invariants of the Calabi-Yau manifold $X$.

One special class of such moduli space studied extensively in $[10 ; 11]$ is when $r=1$ and $I=\mathcal{O}_{X}$. Because $X$ is smooth and $\mathcal{E}$ is stable, it must be torsion free, and be a subsheaf of its double dual $\mathcal{E}^{\vee \vee} \cong \mathcal{O}_{X}$; hence it becomes an ideal sheaf of a subscheme $Z \subset X$. Following the notation of $[10 ; 11]$, after picking a curve class $\beta$ and an integer $n$, we denote by

$$
I_{X}(\beta, n)
$$

the Hilbert scheme of one dimensional subschemes $Z \subset X$ satisfying $[Z]=\beta$ and $\chi\left(\mathcal{O}_{Z}\right)=n$. A simple argument shows that $I_{X}(\beta, n)$ is the moduli space $\mathfrak{M}_{X}\left(0, I, \beta, c_{3}\right)$ with $c_{3}$ the third Chern class of any ideal sheaf of $Z \subset X$ in $I_{X}(\beta, n)$. One special feature of the moduli of rank one torsion free sheaves is that they admit perfect-obstruction theory for all smooth projective threefolds.

Following [10; 11], for Calabi-Yau threefold $X$ and curve $\beta$ one forms the generating function

$$
\mathcal{D} \mathcal{T}_{X, \beta}(q)=\sum_{n} \operatorname{deg}\left[I_{X}(\beta, n)\right]^{\mathrm{vir}} q^{n} .
$$

In case $X$ is any smooth threefold, since the Hilbert schemes $I_{X}(0, n)$ have virtual dimensions zero, one defines $\mathcal{D} \mathcal{T}_{X, \beta}(q)$ according to the same formula and call it the dimensional zero Donaldson-Thomas series as well. Of the several conjectures on $\mathcal{D} \mathcal{T}_{X, \beta}(q)$ proposed in $[10 ; 11]$, one is about the dimension zero Donaldson-Thomas invariants $\mathcal{D} \mathcal{T}_{X, 0}(q)$. Let $M(q)$ be the three dimensional partition function

$$
M(q)=\prod_{n} \frac{1}{\left(1-q^{n}\right)^{n}}
$$

and $c_{3}\left(T_{X} \otimes K_{X}\right)$ be the third Chern class, viewed as the Chern number, of $T_{X} \otimes K_{X}$. 
Conjecture $0.1[10 ; 11]$ For any smooth projective threefold $X$, the dimension zero Donaldson-Thomas series $\mathcal{D} \mathcal{T}_{X, 0}(q)$ has the form

$$
\mathcal{D} \mathcal{T}_{X, 0}(q)=M(-q)^{c_{3}\left(T_{X} \otimes K_{X}\right)} .
$$

In this paper, we shall prove this conjecture for all compact smooth complex threefolds.

Theorem 0.2 The zero-dimensional Donaldson-Thomas series $\mathcal{D} \mathcal{T}_{X, 0}(q)$ for any compact smooth complex threefold $X$ are of the form

$$
\mathcal{D} \mathcal{T}_{X, 0}(q)=M(-q)^{c_{3}\left(T_{X} \otimes K_{X}\right)} .
$$

We remark that this conjecture was independently proved for the class of Calabi-Yau threefolds based on different method by Behrend and Fantechi [3], and for projective threefolds by Levine and Pandharipande [6].

We now briefly outline the proof of this theorem. Clearly, in case $X$ is a disjoint union of two smooth proper threefolds $X_{1}$ and $X_{2}$, then

$$
I_{X}(0, n)=\coprod_{n_{1}+n_{2}=n} I_{X_{1}}\left(0, n_{1}\right) \times I_{X_{2}}\left(0, n_{2}\right) .
$$

Since the deformation of the ideal sheaf of the union $Z_{1} \cup Z_{1} \subset X_{1} \cup X_{2}$ is the direct product of the deformation of $Z_{1} \subset X_{1}$ and the deformation of $Z_{2} \subset X_{2}$, we have

$$
\left[I_{X}(0, n)\right]^{\mathrm{vir}}=\coprod_{n_{1}+n_{2}=n}\left[I_{X_{1}}\left(0, n_{1}\right)\right]^{\mathrm{vir}} \times\left[I_{X_{2}}\left(0, n_{2}\right)\right]^{\mathrm{vir}}
$$

Therefore

$$
\mathcal{D} \mathcal{T}_{X, 0}(q)=\mathcal{D} \mathcal{T}_{X_{1}, 0}(q) \cdot \mathcal{D} \mathcal{T}_{X_{2}, 0}(q)
$$

Put differently, the correspondence that sends any threefold to its zero-dimensional Donaldson-Thomas series defines a homomorphism from the additive semigroup

$$
\mathfrak{P}_{\mathbb{C}}=\{\text { All smooth projective threefolds }\} / \text { iso }
$$

to the multiplicative semigroup of infinite series $\mathbb{Z}[[q]]$.

A distant cousin of complex manifolds are so called weakly complex manifolds, which by definition are smooth compact real manifolds $M$ (possibly with smooth boundaries) together with $\mathbb{C}$-vector bundle structures on the stabilizations ${ }^{1}$ of their tangent bundles $T_{M}$.

\footnotetext{
${ }^{1}$ Here as usual a stabilization of $T_{M}$ is a direct sum of $T_{M}$ with some trivial bundle $\mathbb{R}^{m}$ on $M$; together with the identity $T_{M} \oplus \mathbb{R}^{m}=\left(T_{M} \oplus \mathbb{R}^{m}\right) \oplus \mathbb{R}^{2}$ with the last $\mathbb{R}^{2}$ is given the obvious complex structure $\mathbb{R}^{2} \cong \mathbb{C}$.
} 
The equivalence classes of weakly complex manifolds (without boundaries) modulo the relations $[\partial W]=0$ forms a group, called the complex cobordism group $\Omega^{\mathbb{C}}$, under the addition $\left[M_{1}\right]+\left[M_{2}\right]=\left[M_{1} \bigsqcup M_{2}\right]$. It is a classical result that $\Omega^{\mathbb{C}} \otimes_{\mathbb{Z}} \mathbb{Q}$ is generated by all possible products of projective spaces $\mathbf{P}^{n}$. As a consequence, the six (real) dimensional complex cobordism group $\Omega_{6}^{\mathbb{C}}$ is generated by

$$
Y_{1}=\mathbf{P}^{3}, \quad Y_{2}=\mathbf{P}^{2} \times \mathbf{P}^{1} \quad \text { and } \quad Y_{3}=\left(\mathbf{P}^{1}\right)^{3} .
$$

The crucial step in proving Theorem 0.2 is to establish

Proposition 0.3 There are universal polynomials

$$
f_{0}, f_{1}, f_{2}, \cdots
$$

in Chern numbers of smooth complex threefolds so that for any smooth projective threefold $X$ its zero-dimensional Donaldson-Thomas series are of the form

$$
\mathcal{D} \mathcal{T}_{X, 0}(q)=\sum_{n} f_{n}(X) q^{n}
$$

Because any complex threefold $X$ is $\mathbb{C}$-cobordant to $\frac{m_{1}}{m} Y_{1}+\frac{m_{2}}{m} Y_{2}+\frac{m_{3}}{m} Y_{3}$ for some integers $n_{i}$, knowing the Proposition, and that cobordant weakly complex manifolds have identical Chern numbers,

$$
\sum_{n} f_{n}(m X) q^{n}=\sum_{n} f_{n}\left(m_{1} Y_{1}+m_{2} Y_{2}+m_{3} Y_{3}\right) q^{n} .
$$

By the definition, the left hand side is

$$
\mathcal{D} \mathcal{T}_{m X, 0}(q)=\mathcal{D} \mathcal{T}_{X, 0}(q)^{m}
$$

while the right hand side is

$$
\mathcal{D} \mathcal{T}_{m_{1} Y_{1}+m_{2} Y_{2}+m_{3} Y_{3}, 0}(q)=\mathcal{D} \mathcal{T}_{Y_{1}, 0}(q)^{m_{1}} \cdot \mathcal{D} \mathcal{T}_{Y_{2}, 0}(q)^{m_{2}} \cdot \mathcal{D} \mathcal{T}_{Y_{3}, 0}(q)^{m_{3}} .
$$

Because $Y_{i}$ are toric threefolds, their Donaldson-Thomas series are known $[10 ; 11]$ to have the form

$$
\mathcal{D} \mathcal{T}_{Y_{i}, 0}(q)=M(-q)^{c_{3}\left(T_{Y_{i}} \otimes K_{Y_{i}}\right)} .
$$

Put together, and adding that

$$
m c_{3}\left(T_{X} \otimes K_{X}\right)=\sum_{i=1}^{3} m_{i} c_{3}\left(T_{Y_{i}} \otimes K_{Y_{i}}\right),
$$

we obtain

$$
\mathcal{D} \mathcal{T}_{X, 0}(q)^{m}=M(-q)^{\sum_{i=1}^{3} m_{i} c_{3}\left(T_{Y_{i}} \otimes K_{Y_{i}}\right)}=M(-q)^{m c_{3}\left(T_{X} \otimes K_{X}\right)} .
$$


Finally, because both $\mathcal{D} \mathcal{T}_{X, 0}(q)$ and $M(-q)$ are power series with integer coefficients and constant coefficient one,

$$
\mathcal{D} \mathcal{T}_{X, 0}(q)=M(-q)^{c_{3}\left(T_{X} \otimes K_{X}\right)} .
$$

This would prove the theorem.

As to the proof of the Proposition, we shall first construct a collection of approximations $X^{[\tau]}$ of $X^{[n]}$ indexed by partitions of $[n]=\{1, \cdots, n\}$. To each $X^{[\tau]}$, we shall construct its virtual cycle and prove that its degree can be approximated by the degree of the virtual cycle of $X^{[\sigma]}$ of $\sigma<\tau$, with errors expressible in terms of a universal expression of the Chern numbers of $X$. Thus by induction, we prove that the degree of the virtual cycle of $X^{[n]}$ can also be expressed universally in terms of the Chern numbers of $X$, thus proving the Proposition and the Theorem.

During the early stage of this work, K Behrend developed a theory of micro-local analysis for a symmetric obstruction theory [2]; later, jointly with B Fantechi they proved Conjecture 0.1 for Calabi-Yau threefolds [3]. Toward the end of finalizing this paper, the author was kindly informed by R Pandharipande that he and M Levine have proved the same conjecture using algebraic K-theory [6].

The author also like to take this opportunity to thank Weiping Li for his valuable comments.

\subsection{Terminology}

We shall work with the category of analytic functions in this paper. Thus for any reduced quasi-projective scheme $W$, we shall work with and denote by $\mathcal{O}_{W}$ the sheaf of analytic functions on $W$; we shall use ordinary open subsets of the $W$ unless otherwise stated.

To distinguish from that of analytic spaces, we shall reserve the words schemes and morphisms to mean algebraic schemes and algebraic morphisms, though viewed as objects in analytic category.

Often, we shall work with open subsets of quasi-projective schemes. We shall endow such sets with their reduced induced analytic structures and work with their sheaves of analytic functions. We shall call such space either analytic spaces or analytic schemes. Accordingly, whenever we say two analytic spaces isomorphic we mean that they are isomorphic as analytic spaces.

In this paper, we reserve the word smooth to mean the smoothness in $C^{\infty}$-category. Thus a smooth function is a $C^{\infty}$-function and a smooth map is a $C^{\infty}$-map. 
There is one case in which we need to keep non-reduced scheme structures. It is the case of flat $U$-families of zero-subschemes $\mathcal{Z} \subset U \times Y$ with $U$ an analytic space and $Y$ an open subset of a smooth varieties. In this case, $\mathcal{Z}$ is defined by the ideal sheaf $\mathcal{I}_{\mathcal{Z}} \subset \mathcal{O}_{U \times Y}$; the structure sheaf of $\mathcal{Z}$ is the quotient $\mathcal{O}_{U \times Y} / \mathcal{I}_{\mathcal{Z}}$; we say that $\mathcal{Z}$ is flat over $U$ if $\mathcal{O}_{\mathcal{Z}}$ is flat over $\mathcal{O}_{U}$.

\section{Hilbert schemes of $\alpha$-points}

The purpose of this section is to construct the filtered approximation of $I_{X}(0, n)$ by Hilbert schemes of $\alpha$-points.

For convenience, we let

$$
X^{[n]}=I_{X}(0, n)_{\text {red }}
$$

be the Hilbert scheme $I_{X}(0, n)$ with the reduced scheme structure.

\subsection{The Definition}

We begin with a finite set $\Lambda$ of order $|\Lambda|$; it could be the set of $n$ integers $[n]=$ $\{1, \cdots, n\}$ or a subset of $[n]$. For such $\Lambda$ we follow the convention

$$
X^{\Lambda}=\left\{\left(x_{a}\right)_{a \in \Lambda} \mid x_{a} \in X\right\} .
$$

In case $|\Lambda|=n$, we define

$$
X^{(\Lambda)}=X^{(n)}=S^{n} X \quad \text { and } \quad X^{[\Lambda]}=X^{[n]} .
$$

Using the Hilbert-Chow morphism $h c: X^{[\Lambda]} \rightarrow X^{(\Lambda)}$, we define

$$
X^{\llbracket \Lambda \rrbracket}=X^{[\Lambda]} \times_{X^{(\Lambda)}} X^{\Lambda}
$$

it comes with a tautological projection $X^{\llbracket \Lambda \rrbracket} \rightarrow X^{\Lambda}$. Obviously, according to this definition a closed point of $X^{\llbracket \Lambda \rrbracket}$ is a pair of a 0 -scheme $\xi \in X^{[\Lambda]}$ and a point $\left(x_{a}\right)_{a \in \Lambda} \in X^{\Lambda}$ such that $h c(\xi)=\sum x_{a}$.

We next look at the set $\mathcal{P}_{\Lambda}$ of all partitions of, or equivalence relations on, the set $\Lambda$. In case $\alpha \in \mathcal{P}_{\Lambda}$ has $k$ equivalence classes $\alpha_{1}, \cdots, \alpha_{k}$, we write

$$
\alpha=\left(\alpha_{1}, \cdots, \alpha_{k}\right) .
$$

It has two distinguished elements: one is $\Lambda$ that is the partition with a single equivalence class $\Lambda$; the other is $0_{\Lambda}$ that is the partition whose equivalence classes are all single element sets. The set $\mathcal{P}_{\Lambda}$ has a partial ordering " $\leq$ " defined by

$$
\text { " } \alpha \geq \beta " \Longleftrightarrow " a \sim_{\beta} b \Rightarrow a \sim_{\alpha} b " .
$$


In case $\alpha=\left(\alpha_{1}, \cdots, \alpha_{k}\right)$, then $\alpha \geq \beta$ if and only if $\beta$ is finer than $\alpha$, or that $\beta$ can be written as

$$
\beta=\left(\beta_{11}, \cdots, \beta_{1 l_{1}}, \cdots, \beta_{k 1}, \cdots, \beta_{k l_{k}}\right) \quad \text { so that } \quad \alpha_{i}=\cup_{j=1}^{l_{i}} \beta_{i j} .
$$

Under this partial ordering, the element $\alpha \wedge \beta$, which is defined by

$$
" a \sim_{\alpha \wedge \beta} b " \Longleftrightarrow " a \sim_{\alpha} b \text { and } a \sim_{\beta} b ",
$$

or equivalently for $\beta=\left(\beta_{1}, \cdots, \beta_{l}\right)$ it is $\alpha \wedge \beta=\left(\alpha_{1} \cap \beta_{1}, \cdots, \alpha_{k} \cap \beta_{l}\right)$, is the largest element among all that are less than or equal to both $\alpha$ and $\beta$. Following this rule, $0_{\Lambda}$ is the smallest element and $\Lambda$ is the largest element in $\mathcal{P}_{\Lambda}$.

For $\alpha \in \mathcal{P}_{\Lambda}$, we let $X^{\llbracket \alpha \rrbracket}$ be (reduced) Hilbert scheme of $\alpha$-points in $X$. Let $\alpha$ be as in (1). Because each $\alpha_{i}$ is a set, we can form $X^{\alpha_{i}}, X^{\left(\alpha_{i}\right)}, X^{\left[\alpha_{i}\right]}$ and $X^{\llbracket \alpha_{i} \rrbracket}$ respectively. We then define

$$
X^{(\alpha)}=\prod_{i=1}^{k} X^{\left(\alpha_{i}\right)}, \quad X^{[\alpha]}=\prod_{i=1}^{k} X^{\left[\alpha_{i}\right]}, \quad X^{\llbracket \alpha \rrbracket}=\prod_{i=1}^{k} X^{\llbracket \alpha_{i} \rrbracket} ;
$$

they fit into the Cartesian product:

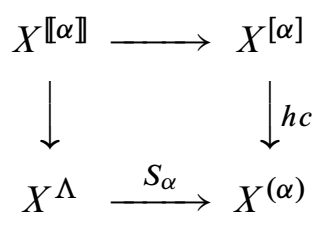

We shall call points in $X^{\llbracket \alpha \rrbracket} \alpha$-zero-subschemes and call $X^{\llbracket \alpha \rrbracket}$ the Hilbert scheme of $\alpha$-points.

The space $X^{\llbracket \alpha \rrbracket}$ coincides with $X^{\llbracket \beta \rrbracket}$ over a large open subset of each. For instance, both $X^{\llbracket 0_{\Lambda} \rrbracket}=X^{\Lambda}$ and $X^{\llbracket \Lambda \rrbracket}$ contains as their open subsets the set of $n=|\Lambda|$ distinct ordered points in $X$. It is when distinct simple points specialize to points with multiplicities the space $X^{\llbracket 0_{\Lambda} \rrbracket}$ becomes different from $X^{\llbracket \Lambda \rrbracket}$ : for the former they remain as simple points by allowing multiple simple points to occupy identical positions in $X$; for the later fat points with non-reduced scheme structures emerge. This way, the collection

$$
\left\{X^{\llbracket \beta \rrbracket} \mid \beta \in \mathcal{P}_{\Lambda}\right\}
$$

forms an increasingly finer approximation of $X^{\llbracket \Lambda \rrbracket}$.

Though the notion of $X^{\llbracket \alpha \rrbracket}$ seems artificial at first, it proves to be useful in keeping tracking of the difference among all $X^{\llbracket \alpha \rrbracket}$. Lastly, in case $\Lambda=[n]$, we shall follow the 
convention

$$
X^{\Lambda}=X^{n}, \quad X^{(\Lambda)}=X^{(n)}, \quad X^{[\Lambda]}=X^{[n]}, \quad X^{\llbracket \Lambda \rrbracket}=X^{\llbracket n \rrbracket} .
$$

\subsection{The relative case}

We can generalize the notion of Hilbert scheme of $\alpha$-points to that of smooth families of varieties. Let

$$
\pi: Y \longrightarrow T
$$

be a smooth family of quasi-projective varieties. For any integer $l$, we let $I_{Y / T}(0, l)$ be the relative Hilbert scheme of length $l 0$-subschemes of fibers of $Y / T$. It is the fine moduli scheme representing the functor parameterizing all flat $S$-families of length $l$ 0 -schemes $Z \subset Y \times_{T} S$. As before, we let

$$
Y^{[l]}=I_{Y / T}(0, l)_{\mathrm{red}}
$$

be $I_{Y / T}(0, l)$ with the reduced scheme structure. The moduli $Y^{[l]}$ is a scheme over $T$ with a universal family. In case for an open $U \subset T$ with $Y \times_{T} U=Y_{0} \times U$, then canonically

$$
Y^{[l]} \times_{T} U \equiv Y_{0}^{[l]} \times U
$$

As before, for any $\alpha \in \mathcal{P}_{\Lambda}$ we let $Y^{\Lambda}, Y^{(\alpha)}, Y^{[\alpha]}$ and $Y^{\llbracket \alpha \rrbracket}$ be the Cartesian products presented before with $X$ replaced by $Y$ and with products replaced by fiber products over the base scheme $T$. Again in case $Y \times_{T} U=Y_{0} \times U$,

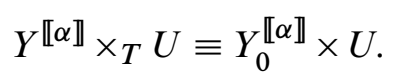

The three cases we shall apply this construction is for the trivial fiber bundle $\mathrm{pr}_{1}: X \times$ $X \rightarrow X$, for the total space of the tangent bundle $T X \rightarrow X$ and for the total space of the universal quotient bundle $Q$ of a Grassmannian $G r=G r(N, 3)$ of quotients $\mathbb{C}^{3}$ of $\mathbb{C}^{N}$. We shall come back to this in detail later.

\subsection{Partial equivalences}

As mentioned before, the collection $Y \llbracket \beta \rrbracket$ forms an increasingly finer approximation of $Y^{\llbracket \Lambda \rrbracket}$. It is the purpose of this subsection to make this precise.

We begin with comparing $Y^{\llbracket \Lambda \rrbracket}$ with $Y^{\llbracket \alpha \rrbracket}$ for an $\alpha=\left(\alpha_{1}, \cdots, \alpha_{k}\right)$. By definition, a point in $Y^{\llbracket \alpha_{i} \rrbracket}$ consists of

$$
\left(\xi_{i},\left(x_{a}\right)_{a \in \alpha_{i}}\right) \in Y^{\left[\alpha_{i}\right]} \times_{T} Y^{\alpha_{i}}
$$

subject to the constraint $h c\left(\xi_{i}\right)=\sum_{a \in \alpha_{i}} x_{a}$. In case the support of $\xi_{i}$ is disjoint from that of $\xi_{j}$, then $\xi_{i} \cup \xi_{j}$ is naturally a zero-subscheme in $Y$ of length $\left|\alpha_{i} \cup \alpha_{j}\right|$; 
the pair $\left(\xi_{i} \cup \xi_{j},\left(x_{a}\right)_{a \in \alpha_{i} \cup \alpha_{j}}\right)$ thus is a point in $Y^{\llbracket \alpha_{i} \cup \alpha_{j} \rrbracket}$. Applying this to all pairs $1 \leq i<j \leq k$, we see that

$$
\left(\cup_{i=1}^{k} \xi_{i},\left(x_{a}\right)_{a \in \Lambda}\right) \in Y^{\llbracket \Lambda \rrbracket}
$$

if and only if the supports $h c\left(\xi_{i}\right)$ are mutually disjoint.

In general, for $\alpha<\beta=\left(\beta_{1}, \cdots, \beta_{l}\right)$ and $1 \leq j \leq l$, the pair

$$
\left(\cup_{\alpha_{i} \subset \beta_{j}} \xi_{i},\left(x_{a}\right)_{a \in \beta_{j}}\right) \in Y^{\llbracket \beta_{j} \rrbracket}
$$

if and only if the supports $\left\{h c\left(\xi_{i}\right) \mid \alpha_{i} \subset \beta_{j}\right\}$ are mutually disjoint. This leads to the definition

Definition 1.1 For $\alpha<\beta$ we define $\Delta_{(\alpha, \beta)}$ be the set

$\left\{x \in Y^{\Lambda} \mid x_{a}=x_{b}\right.$ for at least one pair $a, b \in \Lambda$ so that $a \sim_{\beta} b$ but $\left.a \chi_{\alpha} b\right\} ;$

for general $\alpha \neq \beta$ we define $\Delta_{(\alpha, \beta)}=\Delta_{(\alpha, \alpha \wedge \beta)} \cup \Delta_{(\beta, \alpha \wedge \beta)}$; we define the discrepancy between $Y \llbracket \alpha \rrbracket$ and $Y \llbracket \beta \rrbracket$ be

$$
\Delta_{(\alpha, \beta)}^{\llbracket \alpha \rrbracket} \triangleq Y^{\llbracket \alpha \rrbracket_{Y^{\Lambda}} \Delta_{(\alpha, \beta)} .}
$$

We define

$$
Y_{(\alpha, \beta)}^{\llbracket \alpha \rrbracket}=Y^{\llbracket \alpha \rrbracket}-\Delta_{(\alpha, \beta)}^{\llbracket \alpha \rrbracket} .
$$

Lemma 1.2 Given any pair $\alpha, \beta \in \mathcal{P}_{\Lambda}$, we have a functorial isomorphism

$$
Y_{(\alpha, \beta)}^{\llbracket \alpha \rrbracket} \cong Y_{(\beta, \alpha)}^{\llbracket \beta \rrbracket} .
$$

Here by functorial isomorphisms we mean those that are induced by the universal property of the respective moduli spaces.

Proof of Lemma 1.2 We first prove the case $\alpha=\Lambda$. Let $\beta=\left(\beta_{1}, \cdots, \beta_{l}\right) \in \mathcal{P}_{\Lambda}$ with $m_{i}=\left|\beta_{i}\right|$; let $S=Y_{(\Lambda, \beta)}^{\llbracket \Lambda \rrbracket}$; and let $(\mathcal{W}, \varphi)$ be the tautological family of $S$. By definition, $\varphi: S \rightarrow Y^{\Lambda}$ is the tautological map and $\mathcal{W}$ is a flat $S$-family of length $|\Lambda|$ zero-subschemes in $Y$ whose Hilbert-Chow map $h c_{\mathcal{W}}: S \rightarrow Y^{(\Lambda)}$ coincide with the composite of $\varphi$ and $Y^{\Lambda} \rightarrow Y^{(\Lambda)}$.

Similarly, a $T$-morphism $S \rightarrow Y^{\llbracket \beta \rrbracket}$ is classified by a collection

$$
\left(\mathcal{W}_{1}, \cdots, \mathcal{W}_{l} ; \varphi_{1}, \cdots, \varphi_{l}\right)
$$


of $S$-families $\mathcal{W}_{i} \subset Y \times_{T} S$ in $X^{\left[\beta_{i}\right]}$ and $\varphi_{i}: S \rightarrow Y^{\beta_{i}}$ so that the Hilbert-Chow morphism $h c_{\mathcal{W}_{i}}: S \rightarrow X^{\left[\beta_{i}\right]}$ (of the family $\mathcal{W}_{i}$ ) makes the diagram

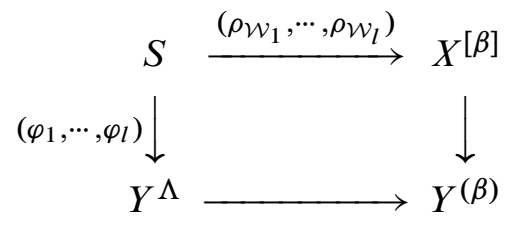

commutative.

To proceed, we shall show that we can split the family $(\mathcal{W}, \varphi)$ into a family as in (2). For each $a \in \Lambda$, we denote by $\varphi_{a}: S \rightarrow Y_{a}$ the $a$-th component of $\varphi$, by $\Gamma_{\varphi_{a}} \subset Y \times_{T} S$ the graph of $\varphi_{a}$ and by $\Gamma_{\beta_{i}}$ the union $\cup_{a \in \beta_{i}} \Gamma_{\varphi_{a}}$. By the definition of $Y_{(\Lambda, \beta)}^{\llbracket \Lambda \rrbracket}$, the set $\Gamma_{\beta_{1}}, \cdots, \Gamma_{\beta_{l}}$ are mutually disjoint closed subsets of $Y \times S$; hence are open and closed subsets of $\Gamma=\cup_{a \in \Lambda} \Gamma_{\varphi_{a}}$.

On the other hand, the closed subscheme $\iota: \mathcal{W} \hookrightarrow Y \times_{T} S$ is set-theoretically identical to $\Gamma$; hence $\mathcal{W}_{i}=\iota^{-1}\left(\Gamma_{\beta_{i}}\right)$ are mutually disjoint open and closed subsets of $\mathcal{W}$, which therefore inherit scheme structures from $\mathcal{W}$ so that $\mathcal{W}=\coprod_{i=1}^{l} \mathcal{W}_{i}$. In particular, they are close subschemes of $Y \times_{T} S$, flat and finite over $S$. Finally, because the fibers of $\mathcal{W}_{i}$ over general closed points in $S$ have length $m_{i}$, by the flatness, $\mathcal{W}_{i}$ is a family of length $m_{i}$ zero-subschemes in $Y$.

Now, because the sets $\Gamma_{\beta_{i}}$ are mutually disjoint, the associated classifying morphisms

$$
\rho_{\mathcal{W}_{i}}: S \longrightarrow Y^{\left[\beta_{i}\right]} \text { and } \varphi_{i}=\prod_{a \in \beta_{i}} \varphi_{a}: S \longrightarrow Y^{\beta_{i}}
$$

satisfies the commutative diagram (3). Therefore, each pair $\left(\mathcal{W}_{i}, \varphi_{i}\right)$ associates to a canonical morphism

$$
\eta_{i}: S \longrightarrow Y^{\left[\beta_{i}\right]} \times_{Y^{\left(\beta_{i}\right)}} Y^{\beta_{i}}=Y^{\llbracket \beta_{i} \rrbracket} .
$$

Put them together, we obtain a morphism

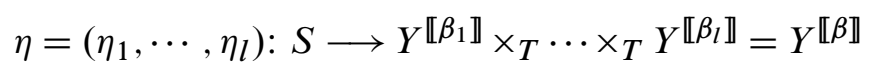

whose image is contained in $Y_{(\beta, \Lambda)}^{\llbracket \beta \rrbracket}$. This way, we have constructed an induced morphism

$$
\eta: S=Y_{(\Lambda, \beta)}^{\llbracket \Lambda \rrbracket} \longrightarrow Y_{(\beta, \Lambda)}^{\llbracket \beta \rrbracket} .
$$

To complete the proof of this special case, we need to construct a morphism

$$
\eta: Y_{(\beta, \Lambda)}^{\llbracket \beta \rrbracket} \longrightarrow S=Y_{(\Lambda, \beta)}^{\llbracket \Lambda \rrbracket}
$$


that is the inverse of $\eta$. But this is straight forward and shall be omitted. This proves the lemma for the case $\alpha=\Lambda$.

For the general case $\alpha=\left(\alpha_{1}, \cdots, \alpha_{k}\right) \in \mathcal{P}_{\Lambda}$, the $\alpha \wedge \beta$ consists of equivalence classes $\alpha_{i} \cap \beta_{j}$, each of order $m_{i j}$. Obviously,

$$
\alpha_{i} \wedge \beta \triangleq\left(\alpha_{i} \wedge \beta_{1}, \cdots, \alpha_{i} \wedge \beta_{l}\right) \in \mathcal{P}_{\alpha_{i}} .
$$

Hence we can apply the proven case of this lemma to conclude

$$
Y_{\left(\alpha_{i} \wedge \beta, \alpha_{i}\right)}^{\llbracket \alpha_{i} \wedge \beta \rrbracket} \cong Y_{\left(\alpha_{i}, \alpha_{i} \wedge \beta\right)}^{\llbracket \alpha_{i} \rrbracket} .
$$

Therefore, because $Y \llbracket \beta \rrbracket=\prod_{T}^{k} Y^{\llbracket \beta_{i} \rrbracket}$, one checks easily that:

$$
\begin{aligned}
Y_{(\alpha, \alpha \wedge \beta)}^{\llbracket \alpha \rrbracket} & =\prod_{i=1}^{k} Y_{\left(\alpha_{i}, \alpha_{i} \wedge \beta\right)}^{\llbracket \alpha_{i} \rrbracket} \cong \prod_{i=1}^{k} Y_{\left(\alpha_{i} \wedge \beta, \alpha_{i}\right)}^{\llbracket \alpha_{i} \wedge \beta \rrbracket} \\
& =\prod_{i=1}^{k}\left(\prod_{j=1}^{l} Y^{\llbracket \alpha_{i} \wedge \beta_{j} \rrbracket}\right) \times_{Y^{\alpha_{i}}}\left(Y^{\alpha_{i}}-\Delta_{\left(\alpha_{i}, \alpha_{i} \wedge \beta\right)}\right) \\
& =\left(\prod_{i, j} Y^{\llbracket \alpha_{i} \wedge \beta_{j} \rrbracket}\right) \times_{Y^{\Lambda}}\left(Y^{\Lambda}-\Delta_{(\alpha, \alpha \wedge \beta)}\right)
\end{aligned}
$$

For the same reason,

$$
Y_{(\beta, \alpha \wedge \beta)}^{\llbracket \beta \rrbracket} \cong\left(\prod_{i, j} Y^{\llbracket \alpha_{i} \wedge \beta_{j} \rrbracket}\right) \times_{Y^{\Lambda}}\left(Y^{\Lambda}-\Delta_{(\beta, \alpha \wedge \beta)}\right) .
$$

Because $\Delta_{(\alpha, \beta)}=\Delta_{(\alpha, \alpha \wedge \beta)} \cup \Delta_{(\beta, \alpha \wedge \beta)}$, we obtain

$$
Y_{(\alpha, \beta)}^{\llbracket \alpha \rrbracket}=Y_{(\alpha, \alpha \wedge \beta)}^{\llbracket \alpha \rrbracket} \times_{Y^{\Lambda}}\left(Y^{\Lambda}-\Delta_{(\alpha, \beta)}\right) \cong Y_{(\beta, \alpha \wedge \beta)}^{\llbracket \beta \rrbracket} \times_{Y^{\Lambda}}\left(Y^{\Lambda}-\Delta_{(\alpha, \beta)}\right)=Y_{(\alpha, \beta)}^{\llbracket \beta \rrbracket} .
$$

This proves the Lemma.

\subsection{Universal families under partial equivalence}

Let $(\alpha, \beta)$ and $\alpha \wedge \beta=\left(\alpha_{1} \cap \beta_{1}, \cdots, \alpha_{k} \cap \beta_{l}\right)$ be as in the proof of the previous lemma; let

$$
\left(\mathcal{W}_{i j}, \varphi_{i j}: i=1, \cdots, k, j=1, \cdots, l\right)
$$

be the universal family of $Y \llbracket \alpha \wedge \beta \rrbracket$. Under the partial equivalence $Y_{(\alpha, \alpha \wedge \beta)}^{\llbracket \alpha \rrbracket} \cong Y_{(\alpha \wedge \beta, \alpha)}^{\llbracket \alpha \wedge \beta \rrbracket}$, the restriction of $\mathcal{W}_{i j}$ to $Y_{(\alpha \wedge \beta, \alpha)}^{\llbracket \alpha \wedge \beta \rrbracket}$ :

$$
\mathcal{W}_{i 1} \times_{Y \llbracket \alpha \wedge \beta \rrbracket} Y_{(\alpha \wedge \beta, \alpha)}^{\llbracket \alpha \wedge \beta \rrbracket}, \cdots, \mathcal{W}_{i l} \times_{Y \llbracket \alpha \wedge \beta \rrbracket} Y_{(\alpha \wedge \beta, \alpha)}^{\llbracket \alpha \wedge \beta \rrbracket},
$$


are families of zero-schemes of $Y$ over $Y_{(\alpha \wedge \beta, \alpha)}^{\llbracket \alpha \rrbracket}$. Following the previous proof, these families, viewed as subschemes in $Y \times_{T} Y_{(\alpha \wedge \beta, \alpha)}^{\llbracket \alpha \wedge \beta \rrbracket}$, are mutually disjoint. Hence their union

$$
\left(\coprod_{j=1}^{l} \mathcal{W}_{i j}\right) \times_{Y \llbracket \alpha \wedge \beta \rrbracket} Y_{(\alpha \wedge \beta, \alpha)}^{\llbracket \alpha \wedge \beta \rrbracket}
$$

forms a flat family of zero schemes in $Y$ of length $\left|\alpha_{i}\right|$ over $Y_{(\alpha \wedge \beta, \alpha)}^{\llbracket \alpha \wedge \beta \rrbracket}$.

Corollary 1.3 Let $\left(\mathcal{Z}_{i}, \varphi_{i} ; i=1, \cdots, k\right)$ be the universal family of $Y^{\llbracket \alpha \rrbracket}$. Then for each $i$,

$$
\left(\coprod_{j=1}^{l} \mathcal{W}_{i j}\right) \times_{Y \llbracket \alpha \wedge \beta \rrbracket} Y_{(\alpha \wedge \beta, \alpha)}^{\llbracket \alpha \wedge \beta \rrbracket}=\mathcal{Z}_{i} \times_{Y \llbracket \alpha \rrbracket} Y_{(\alpha, \alpha \wedge \beta)}^{\llbracket \alpha \rrbracket}
$$

as families of relative zero-subschemes in $Y / T$.

\subsection{Hilbert scheme of centered $\alpha$-points}

In order to parameterize family of slices in $Y^{\llbracket \alpha \rrbracket}$, we need the notion of Hilbert scheme of centered $\alpha$-points.

Let $\pi: Y \rightarrow T$ be the total space of a rank three vector bundle, viewed as a smooth family of affine schemes isomorphic to $\mathbf{A}^{3}$. We let

$$
\varpi: Y^{\Lambda} \longrightarrow V,\left(x_{a}\right)_{a \in \Lambda} \in Y_{t}^{\Lambda} \longmapsto \frac{1}{|\Lambda|} \sum_{a} x_{a} \in Y_{t}
$$

be the fiberwise averaging morphism and let

$$
\varpi^{\llbracket \alpha \rrbracket: Y \llbracket \alpha \rrbracket} \longrightarrow Y
$$

be its composition with the tautological $Y^{\llbracket \alpha \rrbracket} \rightarrow Y^{\Lambda}$. We define the relative Hilbert scheme of centered $\alpha$-points be the preimage of the zero section $0_{Y}$ of $Y$ under $\varpi \llbracket \rrbracket$ :

$$
Y_{0}^{\llbracket \alpha \rrbracket}=Y^{\llbracket \alpha \rrbracket} \times_{Y} 0_{Y}
$$

Intuitively, $Y_{0}^{\llbracket \alpha \rrbracket}$ consists of $\alpha$-zero-subschemes whose center of support lie in the zero section of $Y$.

For any pair $\alpha, \beta \in \mathcal{P}_{\Lambda}$, we define

$$
Y_{0,(\alpha, \beta)}^{\llbracket \alpha \rrbracket}=Y_{0}^{\llbracket \alpha \rrbracket} \cap Y_{(\alpha, \beta)}^{\llbracket \alpha \rrbracket} .
$$


The partial equivalence for $Y \llbracket \alpha \rrbracket$ carries over to

$$
Y_{0,(\alpha, \beta)}^{\llbracket \alpha \rrbracket}=Y_{0,(\beta, \alpha)}^{\llbracket \beta \rrbracket}
$$

\subsection{Outline of the proof}

We now explain briefly the strategy to prove the main Proposition. First, because $X^{\llbracket n \rrbracket}$ is finite over $X^{[n]}$, the degree of its virtual cycle is a fraction of that of $X^{[n]}$. To study the former, we first make sense of the virtual cycles $\left[X^{\llbracket \alpha \rrbracket}\right]^{\text {vir }}$ for all partitions $\alpha$ of $[n]$; we then construct explicitly their cycle representatives $D_{\alpha}$ as cycles in $X^{n}$. Using the isomorphism $X_{(\alpha, \beta)}^{\llbracket \alpha \rrbracket} \cong X_{(\beta, \alpha)}^{\llbracket \beta \rrbracket}$, we can choose $D_{\alpha}$ and $D_{\beta}$ so that their difference lies entirely in a small tubular neighborhood of $\Delta_{(\alpha, \beta)} \subset X^{n}$. Repeating this procedure, we show that the desired degree $\operatorname{deg} D_{[n]}$ is a linear combination of $\operatorname{deg} D_{\alpha}$ plus a discrepancy term which we denote by $\delta_{[n]}$. Using induction on $n$, to prove the main proposition we only need to show that $\delta_{[n]}$ only depend on the Chern numbers of $X$.

To prove the last statement, we shall find a cycle representative of $\delta_{[n]}$ that is entirely contained in a small (tubular) neighborhood of the top diagonal

$$
X_{\Delta}^{\Lambda}=\{(x, \cdots, x) \mid x \in X\} \subset X^{n} .
$$

Once we know this, we shall find a small (tubular) neighborhood $U \subset X^{\llbracket \Lambda \rrbracket}$ of

$$
X_{\Delta}^{\llbracket n \rrbracket}=X^{\llbracket n \rrbracket} \times_{X^{n}} X_{\Delta}^{n}
$$

and a fibration

$$
\pi: U \longrightarrow X
$$

whose homotopy type is determined by one of its fiber $\pi^{-1}(x)$ and the tangent bundle $T X$. Since the homotopy type of the fiber $\pi^{-1}(x)$ is universal (independent of threefold $X$ ), the discrepancy $\delta_{[n]}$ thus only depend on the homotopy type of $T X$. This will lead to a proof that $\delta_{[n]}$, and thus $\operatorname{deg}\left[X^{\llbracket n \rrbracket}\right]^{\text {vir }}$, depends only on a universal expression in Chern numbers of $X$.

\section{Top diagonal of the Hilbert scheme of $\alpha$-points}

In this section, we shall give a smooth parameterization of the normal slices to the top diagonal in the Hilbert scheme $X^{\llbracket \alpha \rrbracket}$. Before doing this, we shall comment on the terminology on stratified spaces and their smooth functions. 


\subsection{Stratifications of singular spaces}

Since we primarily are interested in (reduced) quasi-projective schemes and their open subsets, we shall confine ourselves to their stratifications and functions.

For quasi-projective $W$, we shall only consider stratifications by Zariski locally closed smooth subvarieties. It is known that every quasi-projective scheme admits such stratifications. In case we are given a finite collection $\mathcal{R}$ of Zariski closed subsets of $W$, we can find stratifications $\mathcal{S}$ of $W$ subordinating to $\mathcal{R}$ in the sense that each $R \in \mathcal{R}$ is a union of strata in $\mathcal{S}$. To find a canonical such stratification, we can take the smallest such stratification ${ }^{2}$ among those subordinating to $\mathcal{R}$. In case in addition we are given a morphism of schemes $\pi: C \rightarrow W$, we can find a stratification $\mathcal{S}^{\prime}$ of $C$ and a stratification $\mathcal{S}$ of $W$ so that $\mathcal{S}$ is subordinating to $\mathcal{R}$ and $\pi: C \rightarrow W$ is a stratified map. Again, among all such pairs of stratifications there is one that is the smallest; we call such pair the standard stratification of $C \rightarrow W$ subordinating to $\mathcal{R}$.

The collection $\mathcal{R}$ usually arises from the singular loci of a sheaf $\mathcal{E}$ of $\mathcal{O}_{W}$-modules. To such sheaf $\mathcal{E}$ we associate a collection inductively by letting $R_{0}=W$ and letting $R_{i} \subset R_{i-1}$ be the non-locally free locus of the sheaf of $\mathcal{O}_{R_{i-1}}$-modules $\mathcal{E} \times \mathcal{O}_{W} \mathcal{O}_{R_{i-1}}$. We shall call $\left\{R_{i}\right\}$ the loci of non-locally freeness of $\mathcal{E}$.

We next look at the standard stratification of $Y^{\llbracket \alpha \rrbracket}$ for a smooth family over a smooth $T$. Obviously, in case $U \subset T$ is a Zariski open so that $Y \times_{T} U=Y_{0} \times U$, then strata of the standard stratification of $Y^{\llbracket \alpha \rrbracket} \times_{T} U$ are of the form $S \times U$ for $S$ strata of $Y_{0}^{\llbracket \alpha \rrbracket}$. In case $Y / T$ is the total space of a rank three vector bundle, then $Y_{0}=\mathbf{A}^{3}$ and we know that all strata of $Y_{0}^{\llbracket \alpha \rrbracket}$ are invariant under the symmetry group of $Y_{0}$. This proves

Lemma 2.1 Let $Y / T$ be a smooth Zariski fiber bundle. Then the standard stratification of $Y^{\llbracket \alpha \rrbracket}$ restricts to the standard stratification of $\left(Y_{t}\right)^{\llbracket \alpha \rrbracket}=\left(Y^{\llbracket \alpha \rrbracket}\right)_{t}$.

Another property of the standard stratification of $X^{\llbracket \alpha \rrbracket}$ is as follows.

Lemma 2.2 Let $U_{a} \subset X$ and $U_{b} \subset X$ be two analytic open subset that are biholomorphic to each other, say via $f: U_{a} \longrightarrow U_{b}$. Then pulling back via $f^{*}$ of the universal family $\mathcal{I}$ of $X^{\llbracket \alpha \rrbracket}$ over $U_{b}^{\llbracket \alpha \rrbracket \triangleq X^{\llbracket \alpha \rrbracket}}{{ }_{X^{\Lambda}}} U_{a}^{\Lambda}$ defines a map

$$
\mathcal{F}: U_{b}^{\llbracket \alpha \rrbracket} \longrightarrow U_{a}^{\llbracket \alpha \rrbracket}
$$

that is an isomorphism of (standardly) stratified spaces. This construction also applies to smooth family of quasi-projective varieties over a smooth base.

\footnotetext{
${ }^{2}$ Two stratifications $\mathcal{S}_{1} \leq \mathcal{S}_{2}$ if each strata in $\mathcal{S}_{1}$ is the union of strata in $\mathcal{S}_{2}$.
} 
Here we say a continuous map $f: Z_{1} \rightarrow Z_{2}$ between two stratified spaces preserves stratifications if every stratum of $Z_{1}$ is the preimage of a stratum of $Z_{2}$. We say $f$ is a smooth isomorphism of stratified spaces if $f^{-1}$ exists and both $f$ and $f^{-1}$ are smooth and preserve stratifications of $Z_{1}$ and $Z_{2}$.

Proof Let $\alpha=\left(\alpha_{1}, \cdots, \alpha_{k}\right)$. Because $U_{a}^{\llbracket \alpha \rrbracket}$ is canonically isomorphic to

$$
\prod U^{\alpha_{i}} \times{ }_{U_{a}^{(\alpha)}} U_{a}^{\left[\alpha_{i}\right]}
$$

to prove the lemma it suffices to show that for every $n$ the Hilbert scheme of $n$-points $I_{U_{a}}(0, n) \cong I_{U_{b}}(0, n)$ canonically under the map induced by $f$. But this follows from the universal property of Hilbert schemes.

\subsection{Smooth functions on stratified spaces}

We now define the notion of smooth functions on a stratified space.

Definition 2.3 Let $Z$ be a topological space equipped with a stratification $\mathcal{S}$. A smooth function $f: Z \rightarrow \mathbb{C}$ is a continuous function whose restriction to each stratum is smooth.

We let $W$ be an analytic space and let $\mathcal{E}$ be a sheaf of $\mathcal{O}_{W}$-modules. We let $\mathcal{S}$ be a standard stratification subordinating to the loci of the non-locally freeness of $\mathcal{E}$. To such stratification, we denote by $\mathcal{A}_{\mathcal{S}}$ the sheaf of smooth functions on $W$, and define the sheaf of smooth sections of $\mathcal{E}$ be

$$
\mathcal{A}_{\mathcal{S}}(\mathcal{E})=\mathcal{E} \otimes_{\mathcal{O}_{W}} \mathcal{A}_{\mathcal{S}}
$$

Because $\mathcal{A}_{\mathcal{S}}$ has partition of unity, whenever $0 \rightarrow \mathcal{E}_{2} \rightarrow \mathcal{E} \rightarrow \mathcal{E}_{1} \rightarrow 0$ is an exact sequence of sheaves of $\mathcal{O}_{W}$-modules, then both

$$
\mathcal{A}_{\mathcal{S}}\left(\mathcal{E}_{2}\right) \longrightarrow \mathcal{A}_{\mathcal{S}}(\mathcal{E}) \longrightarrow \mathcal{A}_{\mathcal{S}}\left(\mathcal{E}_{1}\right) \longrightarrow 0
$$

and

$$
\Gamma\left(\mathcal{A}_{\mathcal{S}}\left(\mathcal{E}_{2}\right)\right) \longrightarrow \Gamma\left(\mathcal{A}_{\mathcal{S}}(\mathcal{E})\right) \longrightarrow \Gamma\left(\mathcal{A}_{\mathcal{S}}\left(\mathcal{E}_{1}\right)\right) \longrightarrow 0
$$

are exact.

As usual, for any subset $B \subset W$ and sheaf of $\mathcal{O}_{W}$-modules $\mathcal{E}$, the space of smooth sections of $\mathcal{E}$ over $B$ is the limit

$$
\Gamma\left(B, \mathcal{A}_{\mathcal{S}}(\mathcal{E})\right)=\lim _{B \subset U} \Gamma\left(U, \mathcal{A}_{\mathcal{S}}(\mathcal{E})\right)
$$


taken over all open subsets $U$ containing $B$. Thus each $s$ in $\Gamma\left(B, \mathcal{A}_{\mathcal{S}}(\mathcal{E})\right.$ is a smooth section defined on some open $U$ containing $B$. Further, in case $\widetilde{s} \in \Gamma\left(B^{\prime}, \mathcal{A}_{\mathcal{S}}(\mathcal{E})\right)$ is a section over a larger subset $B^{\prime} \supset B$, we say $s^{\prime}$ extends $s$ if there is an open $U \supset B$ so that both $s$ and $s^{\prime}$ are defined over $U$ and $\left.\left.s\right|_{U} \equiv s^{\prime}\right|_{U}$. Following this convention, the restriction homomorphism

$$
\Gamma\left(B^{\prime}, \mathcal{A}_{\mathcal{S}}(\mathcal{E})\right) \rightarrow \Gamma\left(B, \mathcal{A}_{\mathcal{S}}(\mathcal{E})\right)
$$

is surjective for any pair of sets $B \subset B^{\prime}$ with $B$ closed in $B^{\prime}$,

\subsection{Normal slices to the diagonal in $X \times X$}

We begin with constructing an isomorphism of a tubular neighborhood $\mathcal{U}$ of $\Delta(X) \subset$ $X \times X$ with a tubular neighborhood $\mathcal{V}$ of the zero section $0_{X} \subset T X$. This map will be a smooth isomorphism of analytic fiber bundle $\mathcal{U} / X$ and $\mathcal{V} / X$ in the sense that for each $x \in X$ the fiber $\mathcal{U}_{x}$ and $\mathcal{V}_{x}$ are isomorphic as analytic spaces. Here $\mathcal{U} / X$ is via the first projection $\mathrm{pr}_{1}: \mathcal{U} \subset X \times X \longrightarrow X$.

Since $\mathcal{V}_{x} \subset T_{x} X$, it has a distinguished point $0 \in \mathcal{V}_{x}$ and $T_{0} \mathcal{V}_{x} \equiv T_{x} X$ canonically; likewise, $\mathcal{U}_{x}$ is a neighborhood of $x \in X$, which has its distinguished point $x \in \mathcal{U}_{x}$ and isomorphism $T_{x} \mathcal{U}_{x} \equiv T_{x} X$.

Lemma 2.4 We can find a smooth isomorphism

$$
\varphi: \mathcal{U} \longrightarrow \mathcal{V}
$$

of a tubular neighborhood $\mathcal{U}$ of $\Delta(X) \subset X \times X$ and a tubular neighborhood $\mathcal{V}$ of $0_{X} \subset T X$, both considered as vector bundles over $X$, such that

(1) restricting to each fiber $\mathcal{U}_{x}$ the map $\varphi_{x}=\left.\varphi\right|_{\mathcal{U}_{x}}: \mathcal{U}_{x} \rightarrow \mathcal{V}_{x}$ is a biholomorphism,

(2) $\varphi_{x}(x)=0$ and that $d \varphi_{x}: T_{x} \mathcal{U}_{x} \rightarrow T_{0} \mathcal{V}_{x}$ is the identity map.

Proof First, after identifying $T_{x} V \cong V$ in the standard way for the threefold $V=\mathrm{A}^{3}$ we can define such $\varphi$ globally:

$$
\phi: V \times V \longrightarrow T V ; \quad V \times V \ni(x, v) \longmapsto v-x \in T_{x} V .
$$

Hence for any open $U_{a} \subset X$ that admits an open embedding $U_{a} \subset V$, the map $\phi$ restricting to

$$
\mathcal{U}_{\alpha}=\phi^{-1}\left(T_{U_{a}} V\right) \cap\left(U_{a} \times U_{a}\right) \subset X \times X
$$

is a local version of the map required by the lemma; we denote such $\left.\phi\right|_{\mathcal{U}_{\alpha}}$ by

$$
\varphi_{a}: \mathcal{U}_{a} \subset X \times X \longrightarrow T X .
$$


We next pick an open covering $\left\{U_{a}\right\}$ of $X$ with embedding $U_{a} \subset V$ and a partition of unity $\sum \eta_{a} \equiv 1$ subordinate to the covering $U_{a}$; we form

$$
\mathcal{U} \triangleq\left\{(x, y) \in X \times X \mid(x, y) \in \mathcal{U}_{a} \text { whenever } x \in \operatorname{Supp}\left(\eta_{\alpha}\right)\right\} .
$$

Because $\operatorname{Supp}\left(\eta_{\alpha}\right)$, which is the closure of $\eta_{\alpha} \neq 0$, is compact and is contained in $U_{a}$, $\mathcal{U}$ is open and contains the diagonal $\Delta(X) \subset X \times X$. Over $\mathcal{U}$, we define

$$
\varphi(x, y)=\sum_{\alpha} \eta_{a}(x) \varphi_{a}(x, y) \in T X .
$$

We let $\pi: \mathcal{U} \rightarrow X$ be the projection induced by the second projection $\operatorname{pr}_{2}: X \times X \rightarrow X$. Clearly, restricting to each fiber $\mathcal{U}_{x}=\pi^{-1}(x)$,

$$
\varphi_{x}(\cdot)=\varphi(x, \cdot): \mathcal{U}_{x} \rightarrow T_{x} X
$$

is analytic. Further, because $\varphi_{a}(x, x)=0$ and $\left.d_{y} \varphi_{a}(x, y)\right|_{y=x}=\mathrm{id}$ whenever $\eta_{a}(x) \neq$ 0 , we have $\varphi_{x}(x)=0$ and $d \varphi_{x}: T_{x} \mathcal{U}_{x} \rightarrow T_{0} \mathcal{V}_{x}$ is the identity map. Therefore, by replacing $\mathcal{U}$ by a sufficiently small tubular neighborhood $\mathcal{U}^{\prime}$ of $\Delta(X) \subset X \times X$ and let $\mathcal{V}^{\prime}=\varphi\left(\mathcal{U}^{\prime}\right)$, the restriction $\left.\varphi^{\prime} \triangleq \varphi\right|_{\mathcal{U}^{\prime}}$ becomes the desired map.

\subsection{Induced map on Hilbert schemes}

The map $\varphi$ induces a smooth map from the relative Hilbert scheme $\mathcal{U}^{\llbracket \alpha \rrbracket}$ to $\mathcal{V}^{\llbracket \alpha \rrbracket}$ as fiber bundles over $X$.

We begin with the definition of $\mathcal{U}^{\llbracket \alpha \rrbracket}$ and $\mathcal{V}^{\llbracket \alpha \rrbracket}$. First, by letting $Y=T X$ be the vector bundle over $T=X$, we can apply the previous discussion to form the relative Hilbert scheme of $\alpha$-points $(T X)^{\llbracket \alpha \rrbracket}$. Let $(T X)^{\llbracket \alpha \rrbracket} \rightarrow(T X)^{\Lambda}$ be the tautological map; let $\mathcal{V}^{\Lambda}$ be the product of $|\Lambda|$-copies of $\mathcal{V}$ over $X$ indexed by $\Lambda$, which is an open subset of $(T X)^{\Lambda}$. We define $\mathcal{V}^{\llbracket \alpha \rrbracket}$, called the relative Hilbert scheme of $\alpha$-points in $\mathcal{V} / X$, be an open subset of $(T X)^{\llbracket \alpha \rrbracket}$ defined via the Cartesian product (to the left below)

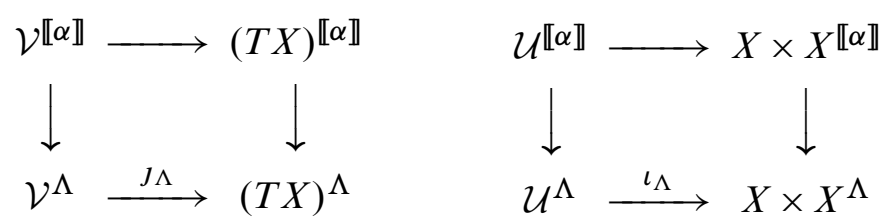

Similarly we form the relative Hilbert scheme $\mathcal{U}^{\llbracket \alpha \rrbracket}$ of $\alpha$-points of $\mathcal{U} / X$ as an open subset of $X \times X^{\llbracket \alpha \rrbracket}$ defined by the square (to the right above). Both $\mathcal{U}^{\llbracket \alpha \rrbracket}$ and $\mathcal{U}^{[\alpha]}$ are analytic spaces over $X$ via the first projection of $X \times X^{\llbracket \alpha \rrbracket}$ and $X \times X^{\Lambda}$.

Because for each closed $x \in X$, the fiber $\varphi_{x}: \mathcal{U}_{x} \rightarrow \mathcal{V}_{x}$ is an isomorphism, restricting to fibers over $x$ the universal families of $\mathcal{U}^{\llbracket \alpha \rrbracket}$ and $\mathcal{V}^{\llbracket \alpha \rrbracket}$ induces a canonical isomorphism 
$\varphi_{x}^{\llbracket \alpha \rrbracket}: \mathcal{U}_{x}^{\llbracket \alpha \rrbracket} \longrightarrow \mathcal{V}_{x}^{\llbracket \alpha \rrbracket}$ from $\mathcal{U}_{x}^{\llbracket \alpha \rrbracket}=\mathcal{U}^{\llbracket \alpha \rrbracket} \times_{X} x$ to $\mathcal{V}_{x}^{\llbracket \alpha \rrbracket}=\mathcal{V}^{\llbracket \alpha \rrbracket} \times_{X} x$. We define

$$
\varphi^{\llbracket \alpha \rrbracket}: \mathcal{U}^{\llbracket \alpha \rrbracket} \longrightarrow \mathcal{V}^{\llbracket \alpha \rrbracket}
$$

be $\varphi_{x}^{\llbracket \alpha \rrbracket}$ when restricting to $\mathcal{U}_{x}^{\llbracket \alpha \rrbracket}$.

Lemma 2.5 The map $\varphi^{\llbracket \alpha \rrbracket}$ is a smooth isomorphism of the analytic fiber bundles $\mathcal{U}^{\llbracket \alpha \rrbracket}$ and $\mathcal{V}^{\llbracket \alpha \rrbracket}$ as stratified spaces. Namely, $\varphi^{\llbracket \alpha \rrbracket}$ preserves the standard stratifications of $\mathcal{V}^{\llbracket \alpha \rrbracket}$ and $\mathcal{U}^{\llbracket \alpha \rrbracket}$ and

$$
\left(\varphi^{\llbracket \alpha \rrbracket}\right)^{-1}\left(\mathcal{A}_{\mathcal{V} \llbracket \alpha \rrbracket}\right)=\mathcal{A}_{\mathcal{U} \llbracket \alpha \rrbracket} .
$$

Further, restricting to fibers over each $x, \varphi^{\llbracket \alpha \rrbracket}$ induces isomorphism of analytic spaces $\mathcal{U}_{x}^{\llbracket \alpha \rrbracket}$ and $\mathcal{V}_{x}^{\llbracket \alpha \rrbracket}$.

Proof The proof is a tautology after applying the universal property of the moduli spaces $\mathcal{U} \llbracket \alpha \rrbracket$ and $\mathcal{V} \llbracket \alpha \rrbracket$. First, because $\varphi: \mathcal{U} \rightarrow \mathcal{V}$ is a smooth isomorphism that preserves the analytic structures of the fibers, pulling back the universal family of $\mathcal{V}^{\llbracket \alpha \rrbracket}$ via $\varphi$ forms a continuous family of relative $\alpha$-schemes of the fiber bundle $\mathcal{U} / X$, thus defines a continuous map

$$
\psi^{\llbracket \alpha \rrbracket:} \mathcal{V}^{\llbracket \alpha \rrbracket} \longrightarrow \mathcal{U}^{\llbracket \alpha \rrbracket} .
$$

Now, let $S \subset \mathcal{V}^{\llbracket \alpha \rrbracket}$ be any stratum of the standard stratification of $\mathcal{V}^{\llbracket \alpha \rrbracket}$. Because its (standard) stratification is induced from that of $(T X)^{\llbracket \alpha \rrbracket}$, the stratum $S$ is the restriction of a stratum $\tilde{S}$ of $(T X)^{\llbracket \alpha \rrbracket}$. By Lemma 2.1, for an $x \in T$ the intersection $\tilde{S} \cap(T X)_{x}^{\llbracket \alpha \rrbracket}$ is a stratum of $\left(T_{x} X\right)^{\llbracket \alpha \rrbracket}$. Hence $S_{x}=S \cap \mathcal{V}_{x}^{\llbracket \alpha \rrbracket}$ is a stratum of $\mathcal{V}_{x}^{\llbracket \alpha \rrbracket}$. Then because $\psi_{x}^{\llbracket \alpha \rrbracket}: \mathcal{V}_{x}^{\llbracket \alpha \rrbracket} \rightarrow \mathcal{U}_{x}^{\llbracket \alpha \rrbracket}$ is an analytic isomorphism, $S_{x}^{\prime}=\psi_{x}^{\llbracket \alpha \rrbracket}\left(S_{x}\right)$ must be a stratum of $\mathcal{U}_{x}^{\llbracket \alpha \rrbracket}$. Similar to the case $T X / X, S_{x}^{\prime}$ is the intersection with $\mathcal{U}_{x}^{\llbracket \alpha \rrbracket}$ of a single stratum $\widetilde{S}^{\prime}$ of $X \times X^{\llbracket \alpha \rrbracket}$. Let $S^{\prime}=\widetilde{S}^{\prime} \cap \mathcal{U}^{\llbracket \alpha \rrbracket}$. Applying Lemma 2.2, we see immediately that

$$
\psi^{\llbracket \alpha \rrbracket}(S)=S^{\prime} .
$$

Thus $\psi \llbracket \alpha \rrbracket$ preserves the stratifications.

On top of this, because restricting to $S$ the pull back of the universal family is a smooth

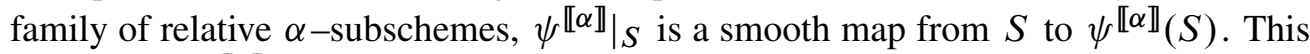
proves that $\psi \llbracket \alpha \rrbracket$ is a smooth stratified map.

Similarly, applying the same argument to $\varphi^{-1}: \mathcal{V} \rightarrow \mathcal{U}$, we obtain the map $\varphi \llbracket \alpha \rrbracket$ that is also a smooth stratified map. Then the composition

$$
\psi^{\llbracket \alpha \rrbracket} \circ \varphi^{\llbracket \alpha \rrbracket}: \mathcal{U}^{\llbracket \alpha \rrbracket} \longrightarrow \mathcal{U}^{\llbracket \alpha \rrbracket}
$$

must be the identity map since it is induced by the identity $\varphi \circ \varphi^{-1}=\mathrm{id}$. For the same reason, $\varphi^{\llbracket \alpha \rrbracket} \circ \psi \llbracket \alpha \rrbracket=$ id as well. This proves that both $\varphi^{\llbracket \alpha \rrbracket}$ and $\psi^{\llbracket \alpha \rrbracket}$ are smooth 
isomorphisms of stratified spaces. Lastly, because $\psi^{\llbracket \alpha \rrbracket}$ preserves fibers over $x$, it defines a smooth map from $\mathcal{V}^{\llbracket \alpha \rrbracket}$ to $\mathcal{U}^{\llbracket \alpha \rrbracket}$ as smooth fiber bundles over $X$.

The last statement is true because restricting to fibers over $x \in X$, both $\varphi^{\llbracket \alpha \rrbracket}$ and $\psi \llbracket \alpha \rrbracket$ are analytic isomorphisms. This proves the Lemma.

\subsection{Top diagonal and its normal slices}

We define the top diagonal $X_{\Delta}^{\llbracket \alpha \rrbracket} \subset X^{\llbracket \alpha \rrbracket}$ be

$$
X_{\Delta}^{\llbracket \alpha \rrbracket}=X_{\Delta}^{\Lambda} \times_{X^{\Lambda}} X^{\llbracket \alpha \rrbracket} \quad \text { where } \quad X_{\Delta}^{\Lambda}=\left\{(x, \cdots, x) \in X^{\Lambda} \mid x \in X\right\} .
$$

Because $X_{\Delta}^{\Lambda} \cong X$, the top diagonal is fibred over $X$ :

$$
X_{\Delta}^{\llbracket \alpha \rrbracket} \longrightarrow X .
$$

The purpose of this subsection is to find an open neighborhood $U$ of $X_{\Delta}^{\llbracket \alpha \rrbracket} \subset X^{\llbracket \alpha \rrbracket}$ and a fiber bundle map $U \rightarrow X$ extending the map (10).

We let $(T X)_{0}^{\llbracket \alpha \rrbracket}$ be the relative Hilbert scheme of centered $\alpha$-points of the fiber bundle $T X / X$. Because $\mathcal{V}^{\llbracket \alpha \rrbracket}$ is an open subbundle of $(T X)^{\llbracket \alpha \rrbracket}$, we define the relative Hilbert scheme of centered $\alpha$-points of $\mathcal{V} / X$ be

$$
\mathcal{V}_{0}^{\llbracket \alpha \rrbracket}=\mathcal{V}^{\llbracket \alpha \rrbracket} \cap(T X)_{0}^{\llbracket \alpha \rrbracket} .
$$

There is another way to define this space. Let $(T X)_{0}^{\Lambda}=(T X)^{\Lambda} \times_{T X} 0_{T X}$ and let $\mathcal{V}_{0}^{\Lambda}$ be the intersection $\mathcal{V}^{\Lambda} \cap(T X)_{0}^{\Lambda}$. Then $\mathcal{V}_{0}^{\llbracket \alpha \rrbracket}$ and $\mathcal{V}_{0}^{\Lambda}$ fit into the following commutative Cartesian squares

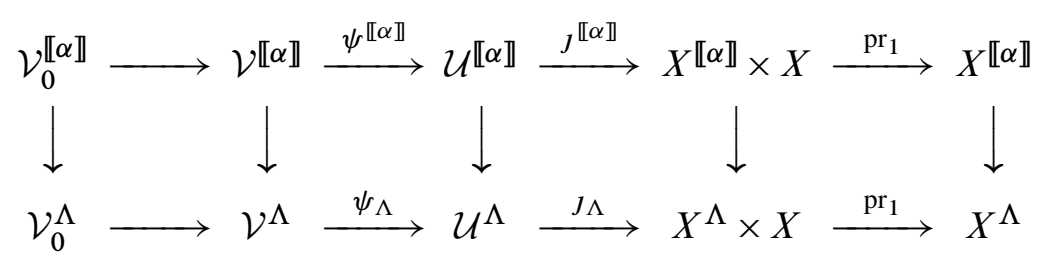

in which the $\psi_{\Lambda}, J_{\Lambda}$ and $J^{\llbracket \alpha \rrbracket}$ are the obvious maps induced by $\psi$ and by $J: \mathcal{U} \rightarrow$ $X \times X$. We let $\Psi_{\alpha}: \mathcal{V}_{0}^{\llbracket \alpha \rrbracket} \rightarrow X^{\llbracket \alpha \rrbracket}$ be the composite of the arrows in the top line.

Lemma 2.6 After shrinking $\mathcal{V}$ if necessary, the image set

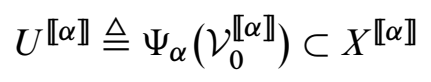


is an open neighborhood of $X_{\Delta}^{\llbracket \alpha \rrbracket} \subset X^{\llbracket \alpha \rrbracket}$. Further, after endowing $U$ with the induced stratification through the inclusion $U^{\llbracket \alpha \rrbracket} \subset X^{\llbracket \alpha \rrbracket}$, the induced map

$$
\Psi_{\alpha}: \mathcal{V}_{0}^{\llbracket \alpha \rrbracket} \longrightarrow U^{\llbracket \alpha \rrbracket}
$$

becomes a smooth isomorphism of stratified spaces.

Proof Because each square in the above diagram is a Cartesian product square, to prove that $\Psi_{\alpha}\left(\mathcal{V}_{0}^{\llbracket \alpha \rrbracket}\right)$ is open in $X^{\llbracket \alpha \rrbracket}$ it suffices to show that the image of $\mathcal{V}_{0}^{\Lambda}$ in $X^{\Lambda}$ under the composite of the bottom line is open in $X^{\Lambda}$.

We let $h: \mathcal{V}_{0}^{\Lambda} \rightarrow X^{\Lambda}$ be the composite of the bottom line in the above diagram. We first prove that the differential

$$
d h(\xi): T_{\xi} \mathcal{V}_{0}^{\Lambda} \longrightarrow T_{h(\xi)} X^{\Lambda}
$$

is an isomorphism at each $\xi$ in the zero section $0_{(T X)^{\Lambda}} \subset(T X)^{\Lambda}$.

Let $\xi \in 0_{(T X)^{\Lambda}}$ be a point over $x \in X$. Since $\left(T_{X} X\right)_{0}^{\Lambda}$ intersects $0_{(T X)^{\Lambda}}$ transversal at $\xi$, the tangent space $T_{\xi} \mathcal{V}_{0}^{\Lambda}$ is a direct sum of $T_{\xi}\left(T_{X} X\right)_{0}^{\Lambda}$ and $T_{\xi} 0_{(T X)^{\Lambda}}$. Clearly, the images $d h\left(T_{\xi}\left(T_{X} X\right)_{0}^{\Lambda}\right)$ and $d h\left(T_{\xi} 0_{(T X)^{\Lambda}}\right)$ are

$$
\left\{\left(v_{a}\right)_{a \in \mathcal{P}_{\Lambda}} \in\left(T_{x} X\right)^{\Lambda} \mid \sum v_{a}=0\right\} \quad \text { and } \quad\left\{(v, \cdots, v) \in\left(T_{x} X\right)^{\Lambda} \mid v \in T_{x} X\right\}
$$

respectively. Thus $d h$ is an isomorphism at $\xi \in 0_{(T X)^{\Lambda}} \subset \mathcal{V}_{0}^{\Lambda}$ and thus $h$ is a diffeomorphism near $0_{(T X)^{\Lambda}} \subset \mathcal{V}_{0}^{\Lambda}$. In particular, if we shrink $\mathcal{V}$ if necessary, $h$ becomes a diffeomorphism from $\mathcal{V}_{0}^{\Lambda}$ to the image $h\left(\mathcal{V}_{0}^{\Lambda}\right)$, an open neighborhood of $X_{\Delta}^{\Lambda} \subset X^{\Lambda}$.

It follows then that $U^{\llbracket \alpha \rrbracket}=\Psi_{\alpha}\left(\mathcal{V}_{0}^{\llbracket \alpha \rrbracket}\right)$ is an open neighborhood of $X_{\Delta}^{\llbracket \alpha \rrbracket} \subset X^{\llbracket \alpha \rrbracket}$ and the induced map $\Psi_{\alpha}$ is continuous and is one-one and onto. We endow $U \llbracket \alpha \rrbracket=\Psi_{\alpha}\left(\mathcal{V}_{0}^{\llbracket \alpha \rrbracket}\right)$ the stratification induced from that of $X^{\llbracket \alpha \rrbracket}$.

We now prove that $\Psi_{\alpha}$ is a smooth isomorphism of stratified spaces. We first prove that $\Psi_{\alpha}$ preserves the stratifications. Indeed, because all but the furthest left arrow in the top line of the above diagram preserves stratifications, to prove the claim we only need to show that the inclusion $\mathcal{V}_{0}^{\llbracket \alpha \rrbracket} \subset \mathcal{V}^{\llbracket \alpha \rrbracket}$ preserves stratifications, which follows from that the inclusion $(T X)_{0}^{\llbracket \alpha \rrbracket} \rightarrow(T X)^{\llbracket \alpha \rrbracket}$ preserves stratifications. For the later, because $(T X)_{0}^{\llbracket \alpha \rrbracket}=(T X)^{\llbracket \alpha \rrbracket} \times_{T X} 0_{(T X)^{\Lambda}}$ with $\varpi:(T X)^{\llbracket \alpha \rrbracket} \rightarrow T X$ the fiberwise averaging morphism, we only need to prove that the restriction of $\varpi$ to each stratum $S \subset(T X)^{\llbracket \alpha \rrbracket}$

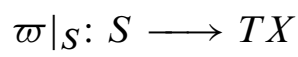


is a submersion. But this is clear since the stratum $S$ is induced by a stratum $S_{0}$ of $\left(T_{X} X\right)^{\llbracket \alpha \rrbracket}$, as shown in Lemma 2.1, and the stratum $S_{0}$ is invariant under the translation group of $T_{X} X$. Therefore $\left.\varpi\right|_{S}$ is a submersion.

Once we know that $\Psi_{\alpha}: \mathcal{V}_{0}^{\llbracket \alpha \rrbracket} \rightarrow X^{\llbracket \alpha \rrbracket}$ preserves stratifications, the fact that each squares above is a Cartesian product shows immediately that it is smooth, and its restriction to each stratum is a diffeomorphism onto its image. Hence, $\Psi_{\alpha}$ is a smooth isomorphism of stratified spaces.

From the definition, $(T X)_{0}^{\llbracket \alpha \rrbracket} \subset(T X)^{\llbracket \alpha \rrbracket}$ is a subscheme, hence $\mathcal{V}_{0}^{\llbracket \alpha \rrbracket} \subset \mathcal{V}^{\llbracket \alpha \rrbracket}$ is an analytic subscheme. Therefore, restricting to fibers $\mathcal{U}_{0, x}^{\llbracket \alpha \rrbracket}$ of $\mathcal{U}_{0}^{\llbracket \alpha \rrbracket}$ over $x \in X$ the map $\Phi$ is analytic.

Before we close this subsection, we shall comment on the partial equivalence of $\mathcal{V}_{0}^{\llbracket \alpha \rrbracket}$ and of $U^{\llbracket \alpha \rrbracket}$. For this, we define

$$
\mathcal{V}_{0,(\alpha, \beta)}^{\llbracket \alpha \rrbracket}=\mathcal{V}_{0}^{\llbracket \alpha \rrbracket} \cap(T X)_{0,(\alpha, \beta)}^{\llbracket \alpha \rrbracket} \quad \text { and } \quad U_{(\alpha, \beta)}^{\llbracket \alpha \rrbracket}=U^{\llbracket \alpha \rrbracket} \cap X_{(\alpha, \beta)}^{\llbracket \alpha \rrbracket} .
$$

Lemma 2.7 Let $\Psi_{\alpha}: \mathcal{V}_{0}^{\llbracket \alpha \rrbracket} \rightarrow U^{\llbracket \alpha \rrbracket}$ be the smooth isomorphism constructed before. Then $\Psi_{\alpha}\left(\mathcal{V}_{0,(\alpha, \beta)}^{\llbracket \alpha \rrbracket}\right)=U_{(\alpha, \beta)}^{\llbracket \alpha \rrbracket}$ and the partial equivalence of $\mathcal{V}_{0}^{\llbracket \alpha \rrbracket}$ and of $U^{\llbracket \alpha \rrbracket}$ are compatible under $\Psi_{\alpha}$.

Proof This is obvious and will be omitted.

\subsection{Universal family of Hilbert schemes of centered $\alpha$-points}

We let $Q \rightarrow G r$ be the total space of the universal quotient rank three vector bundle over the Grassmannian $G r=G r\left(\mathbb{C}^{N}, \mathbb{C}^{3}\right)$ and let $\rho: Q_{0}^{\llbracket \alpha \rrbracket} \rightarrow G r$ be the associated relative Hilbert scheme of centered $\alpha$-points. Let

$$
g: X \longrightarrow G r
$$

be a smooth map so that $T X \cong g^{*} Q$ as smooth vector bundles. To such $g$, we define the pull back

$$
g^{*} Q_{0}^{\llbracket \alpha \rrbracket}=Q_{0}^{\llbracket \alpha \rrbracket} \times_{G r} X=\coprod_{x \in X} \rho^{-1}(g(x)) \subset Q_{0}^{\llbracket \alpha \rrbracket} \times X .
$$

Further, the universal family of $Q_{0}^{\llbracket \alpha \rrbracket}$ pulls back under $g$ to a continuous family of relative centered $\alpha$-points of $T X / X$, thus defines a continuous map over $X$

$$
g_{\alpha}: g^{*} Q_{0}^{\llbracket \alpha \rrbracket} \longrightarrow(T X)_{0}^{\llbracket \alpha \rrbracket} .
$$


Lemma 2.8 This map is a smooth isomorphism of fiber bundles over $X$. Further, its restriction to each fiber is an analytic isomorphism and preserves the partial equivalences of $Q_{0}^{\llbracket \alpha \rrbracket}$ and of $(T X)_{0}^{\llbracket \alpha \rrbracket}$.

\section{Obstruction sheaves}

Our next step is to investigate the obstruction theory of the relative Hilbert scheme of $\alpha$-points of a smooth family $Y / T$. Because $Y^{\Lambda} \rightarrow Y^{(\alpha)}$ is not étale, the defining square

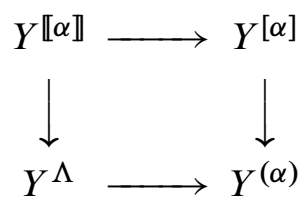

does not allow us to lift the obstruction theory of $Y^{[\alpha]}$ to that of $Y^{\llbracket \alpha \rrbracket}$. Our solution is to take the pull-back of the obstruction sheaf and the normal cone of $Y^{[\alpha]}$ as the obstruction sheaf and normal cone of $Y \llbracket \alpha \rrbracket$. To force $Y \llbracket \alpha \rrbracket \rightarrow Y^{[\alpha]}$ flat, we shall view $Y^{\Lambda} \rightarrow Y^{(\alpha)}$, and hence $Y^{\llbracket \alpha \rrbracket} \rightarrow Y^{[\alpha]}$, as a morphism between stacks.

We will investigate the obstruction sheaves in this section, deferring normal cones to the next section. We begin with a brief account of the relevant extension sheaves.

\subsection{A brief account of extensions sheaves}

Let $Y / T$ be a smooth family of quasi-projective threefolds over a smooth variety $T$; let $S$ be any scheme over $T$ and $Z$ be a flat $S$-family of $0-$ subschemes in $Y_{S}=Y \times_{T} S$; and let $p_{S}: Y_{S} \rightarrow S$ be the projection. Our first goal is to prove a canonical isomorphism relating the traceless part of the relative extension sheaf of the ideal sheaf $\mathcal{I}_{Z}$ of $Z \subset Y_{S}$ with the high direct image sheaf of an extension sheaf.

Lemma 3.1 Let the notation be as before, then we have canonical isomorphism

$$
\mathcal{E x t}_{p_{S}}^{2}\left(\mathcal{I}_{Z}, \mathcal{I}_{Z}\right)_{0} \cong p_{S *} \mathcal{E x t}_{\mathcal{O}_{Y_{S}}}^{3}\left(\mathcal{O}_{Z}, \mathcal{I}_{Z}\right)
$$

Proof We begin with the defining exact sequence

$$
0 \longrightarrow \mathcal{I}_{Z} \longrightarrow \mathcal{O}_{Y_{S}} \longrightarrow \mathcal{O}_{Z} \longrightarrow 0
$$

and its associated long exact sequence of relative extension sheaves

$$
\longrightarrow \mathcal{E} x t_{p_{S}}^{2}\left(\mathcal{O}_{Y_{S}}, \mathcal{I}_{Z}\right) \stackrel{\phi_{2}}{\longrightarrow} \mathcal{E} x t_{p_{S}}^{2}\left(\mathcal{I}_{Z}, \mathcal{I}_{Z}\right) \longrightarrow \mathcal{E} x t_{p_{S}}^{3}\left(\mathcal{O}_{Z}, \mathcal{I}_{Z}\right) \longrightarrow
$$




$$
\longrightarrow \mathcal{E x t}_{p_{S}}^{3}\left(\mathcal{O}_{Y_{S}}, \mathcal{I}_{Z}\right) \stackrel{\phi_{3}}{\longrightarrow} \mathcal{E} x t_{p_{S}}^{3}\left(\mathcal{I}_{Z}, \mathcal{I}_{Z}\right) \longrightarrow 0
$$

Because $\mathcal{O}_{Y_{S}}$ is locally free and $Z$ is a flat family of zero-subschemes $Y_{S}$,

$$
\mathcal{E} x t_{p_{S}}^{k}\left(\mathcal{O}_{Y_{S}}, \mathcal{I}_{Z}\right)=R^{k} p_{S *} \mathcal{I}_{Z}=R^{k} p_{S *} \mathcal{O}_{Y_{S}}, \quad k \geq 2
$$

On the other hand, it follows from the definition that $R^{k} p_{S *} \mathcal{O}_{Y_{S}}$ is a subsheaf of $\mathcal{E x t}_{p_{S}}^{k}\left(\mathcal{I}_{Z}, \mathcal{I}_{Z}\right)$ and the composite

$$
R^{k} p_{S *} \mathcal{O}_{Y_{S}} \stackrel{\subset}{\longrightarrow} \mathcal{E x t}_{p_{S}}^{k}\left(\mathcal{I}_{Z}, \mathcal{I}_{Z}\right) \stackrel{\operatorname{tr}}{\longrightarrow} R^{k} p_{S *} \mathcal{O}_{Y_{S}}
$$

is multiplying by 1 , which is the rank of $\mathcal{I}_{Z}$. Thus $R^{k} p_{S *} \mathcal{O}_{Y_{S}}$ is the trace part of $\mathcal{E x t}_{p_{S}}^{k}\left(\mathcal{I}_{Z}, \mathcal{I}_{Z}\right)$, and the traceless part

$$
\mathcal{E} x t_{p_{S}}^{2}\left(\mathcal{I}_{Z}, \mathcal{I}_{Z}\right)_{0} \cong \mathcal{E} x t_{p_{S}}^{3}\left(\mathcal{O}_{Z}, \mathcal{I}_{Z}\right)
$$

canonically.

To complete the proof of the lemma, we shall apply the local to global spectral sequence. First because the extension sheaf $\mathcal{E} x t^{i}\left(\mathcal{O}_{Z}, \mathcal{I}_{Z}\right)$ is zero away from $Z$ and $Z \rightarrow S$ has relative dimension 0 ,

$$
R^{k} p_{S *} \mathcal{E} x t^{3-k}\left(\mathcal{O}_{Z}, \mathcal{I}_{Z}\right)=0
$$

except when $k=0$. Hence the spectral sequence

$$
R^{\bullet} p_{S *} \mathcal{E x t}{ }^{\bullet}\left(\mathcal{O}_{Z}, \mathcal{I}_{Z}\right) \Rightarrow \mathcal{E} x t_{p_{S}}^{\bullet}\left(\mathcal{O}_{Z}, \mathcal{I}_{Z}\right)
$$

degenerates to

$$
\mathcal{E} x t_{p_{S}}^{3}\left(\mathcal{O}_{Z}, \mathcal{I}_{Z}\right)_{0} \cong p_{S *} \mathcal{E} x t^{3}\left(\mathcal{O}_{Z}, \mathcal{I}_{Z}\right) .
$$

This proves the Lemma.

The relative extension sheaves satisfy the base change property. Let $\rho: S^{\prime} \rightarrow S$ be a morphism and $Z^{\prime}=Z \times_{S} S^{\prime}$ be the subscheme of $Y_{S^{\prime}}=Y_{S} \times{ }_{S} S^{\prime}$, then

$$
\mathcal{E x t}_{p_{S^{\prime}}}^{2}\left(\mathcal{I}_{Z^{\prime}}, \mathcal{I}_{Z^{\prime}}\right)_{0} \cong \rho^{*} \mathcal{E} x t_{p_{S}}^{2}\left(\mathcal{I}_{Z}, \mathcal{I}_{Z}\right)_{0}
$$

and such isomorphisms are compatible with $S^{\prime \prime} \rightarrow S^{\prime} \rightarrow S$.

To prove the base change property, we will apply the Lemma and prove the base change property of

$$
p_{S *} \mathcal{E} x t^{3}\left(\mathcal{O}_{Z}, \mathcal{I}_{Z}\right)
$$

Because $\operatorname{dim} Y / T=3$ and $\mathcal{O}_{Z}$ is flat over $S$, the later admits a length three locally free resolution as a sheaf of $\mathcal{O}_{Y_{S}}$-modules. Then because tensoring this resolution by 
$\mathcal{O}_{S^{\prime}}$ provides a locally free resolution of $\mathcal{O}_{Z^{\prime}}$, applying the definition of extension sheaves we readily see that

$$
\mathcal{E} x t_{\mathcal{O}_{Y_{S^{\prime}}}}^{3}\left(\mathcal{O}_{Z^{\prime}}, \mathcal{I}_{Z^{\prime}}\right) \cong \mathcal{E} x t_{\mathcal{O}_{Y_{S}}}^{3}\left(\mathcal{O}_{Z}, \mathcal{I}_{Z}\right) \otimes_{\mathcal{O}_{S}} \mathcal{O}_{S^{\prime}}
$$

Because the first sheaf if a finite sheaf of $\mathcal{O}_{S^{\prime}}$-modules,

$$
p_{S^{\prime} *} \mathcal{E x t}_{\mathcal{O}_{Y_{S^{\prime}}}}^{3}\left(\mathcal{O}_{Z^{\prime}}, \mathcal{I}_{Z^{\prime}}\right)=\mathcal{E} x t_{\mathcal{O}_{Y_{S^{\prime}}}}^{3}\left(\mathcal{O}_{Z^{\prime}}, \mathcal{I}_{Z^{\prime}}\right)
$$

when the later is viewed as sheaf of $\mathcal{O}_{S^{\prime}}$-modules. Thus by viewing the other $\mathcal{E} x t^{3}$ sheaf as a sheaf of $\mathcal{O}_{S}$-modules, the identity is exactly the base change property (16).

Corollary 3.2 Let $Z \subset Y_{S}$ be as in the previous lemma. Suppose further that $Z=Z_{1} \cup Z_{2}$ is a union of two disjoint flat $S$-families of 0 -subschemes. Then

$$
\mathcal{E}_{x t_{p_{S}}}^{2}\left(\mathcal{I}_{Z}, \mathcal{I}_{Z}\right)_{0} \cong \mathcal{E} x t_{p_{S}}^{2}\left(\mathcal{I}_{Z_{1}}, \mathcal{I}_{Z_{1}}\right)_{0} \oplus \mathcal{E} x t_{p_{S}}^{2}\left(\mathcal{I}_{Z_{2}}, \mathcal{I}_{Z_{2}}\right)_{0}
$$

canonically.

Proof This is true because

$$
\mathcal{E} x t_{p_{S}}^{2}\left(\mathcal{I}_{Z}, \mathcal{I}_{Z}\right)_{0} \cong p_{S *} \mathcal{E} x t^{3}\left(\mathcal{O}_{Z}, \mathcal{I}_{Z}\right) \cong \bigoplus_{i=1}^{2} p_{S *} \mathcal{E} x t^{3}\left(\mathcal{O}_{Z_{i}}, \mathcal{I}_{Z_{i}}\right)
$$

\subsection{Obstruction sheaves of Hilbert scheme of points}

For a smooth family of quasi-projective threefolds over a smooth base $T$, the relative Hilbert scheme $I_{Y / T}(0, n)$ is a quasi-project fine moduli scheme with universal family

$$
\mathcal{Z} \subset Y \times_{T} I_{Y / T}(0, n) ;
$$

its obstruction theory as moduli of stable sheaves with fixed determinants is perfect with obstruction sheaf the traceless part of the relative extension sheaf under the second projection of $Y \times_{T} I_{Y / T}(0, n)$ :

$$
\mathcal{O} b_{I_{Y / T}(0, n)}=\mathcal{E} x t_{\mathrm{pr}_{2}}^{2}\left(\mathcal{I}_{\mathcal{Z}}, \mathcal{I}_{\mathcal{Z}}\right)_{0} .
$$

For $Y^{[n]}$, which is $I_{Y / T}(0, n)$ endowed with the reduced scheme structure, we shall take the pull back of the obstruction sheaf of $I_{Y / T}(0, n)$ under the inclusion $Y^{[n]} \rightarrow$ $I_{Y / T}(0, n)$ as its obstruction sheaf. By the base change property just proved, it can also be expressed as the traceless part of the relative extension sheaf of the universal ideal sheaf of $Y^{[n]}$.

Now let $\alpha=\left(\alpha_{1}, \cdots, \alpha_{k}\right)$ be any element in $\mathcal{P}_{\Lambda}$ as before; let $\left(\mathcal{Z}_{1}, \cdots, \mathcal{Z}_{k}\right)$ be the universal family of $Y^{[\alpha]}$, each is a flat family of length $\left|\alpha_{i}\right|$ zero-subschemes in $Y / T$ 
over $Y^{[\alpha]}$; we let $\mathcal{I}_{\mathcal{Z}_{i}}$ be the ideal sheaf of $\mathcal{Z}_{i} \subset Y \times_{T} Y^{[\alpha]}$. Because $Y^{[\alpha]}=\prod_{T} Y^{\left[\alpha_{i}\right]}$, we define the obstruction sheaf of $Y^{[\alpha]}$ be the direct sum of the pull back of that of $Y^{\left[\alpha_{i}\right]}$, which by Corollary 3.2 is of the form

$$
\mathcal{O} b_{Y}^{[\alpha]}=\bigoplus_{i=1}^{k} \mathcal{E} x t_{\mathrm{pr}_{2}}^{2}\left(\mathcal{I}_{\mathcal{Z}_{i}}, \mathcal{I}_{\mathcal{Z}_{i}}\right)_{0} .
$$

As to $Y^{\llbracket \alpha \rrbracket}$, we shall take the pull-back $\phi_{\alpha}^{*} \mathcal{O} b^{[\alpha]}$ (of the tautological $\phi_{\alpha}: Y^{\llbracket \alpha \rrbracket} \rightarrow Y^{[\alpha]}$ ) as its obstruction sheaf. For the same reason, they can be defined using relative extension sheaves. Let $\left(\mathcal{W}_{1}, \cdots, \mathcal{W}_{k}\right)$ with $\mathcal{W}_{i} \subset Y \times_{T} Y \llbracket \alpha \rrbracket$ be part of the universal family of $Y^{\llbracket \alpha \rrbracket}$; each $\mathcal{W}_{i}$ is the pull back of $\mathcal{Z}_{i}$ under $Y^{\llbracket \alpha \rrbracket} \rightarrow Y^{[\alpha]}$; let $\mathcal{I}_{\mathcal{W}_{i}}$ be the ideal sheaf of $\mathcal{W}_{i} \subset Y \times_{T} Y^{\llbracket \alpha \rrbracket}$. The obstruction sheaf of $Y^{\llbracket \alpha \rrbracket}$ is

$$
\mathcal{O} b_{Y}^{\llbracket \alpha \rrbracket} \equiv \bigoplus_{i=1}^{k} \mathcal{E} x t_{\mathrm{pr}_{2}}^{2}\left(\mathcal{I}_{\mathcal{W}_{i}}, \mathcal{I}_{\mathcal{W}_{i}}\right)_{0}
$$

\subsection{Comparing obstruction sheaves under equivalences}

Our next task is to compare the sheaves $\mathcal{O} b_{Y}^{\llbracket \alpha \rrbracket}$ with $\mathcal{O} b_{Y}^{\llbracket \beta \rrbracket}$ over the partial equivalence $Y_{(\alpha, \beta)}^{\llbracket \alpha \rrbracket} \cong Y_{(\beta, \alpha)}^{\llbracket \beta \rrbracket}$.

Lemma 3.3 Under the partial equivalence $Y_{(\alpha, \beta)}^{\llbracket \alpha \rrbracket} \cong Y_{(\beta, \alpha)}^{\llbracket \beta \rrbracket}$, the restriction to $Y_{(\alpha, \beta)}^{\llbracket \alpha \rrbracket}$ of the obstruction sheaf $\mathcal{O} b_{Y}^{\llbracket \alpha \rrbracket}$ is canonically isomorphic to the restriction to $Y_{(\beta, \alpha)}^{\llbracket \beta \rrbracket}$ of $\mathcal{O} b_{Y}^{\llbracket \beta \rrbracket}$.

Proof We first show that the Lemma can be reduced to the case where $\alpha \geq \beta$. Indeed, because $Y_{(\alpha, \beta)}^{\llbracket \alpha \rrbracket} \cong Y_{(\beta, \alpha)}^{\llbracket \beta \rrbracket}$ is induced by $Y_{(\alpha, \beta)}^{\llbracket \alpha \rrbracket} \subset Y_{(\alpha, \alpha \wedge \beta)}^{\llbracket \alpha \rrbracket} \cong Y_{(\alpha \wedge \beta, \alpha)}^{\llbracket \alpha \wedge \beta \rrbracket}$, should the lemma hold for $\alpha \geq \beta$, we would have

$$
\left.\left.\mathcal{O} b_{Y}^{\llbracket \alpha \rrbracket}\right|_{Y_{(\alpha, \alpha \wedge \beta)}^{\llbracket \alpha \rrbracket}} \cong \mathcal{O} b_{Y}^{\llbracket \alpha \wedge \beta \rrbracket}\right|_{Y_{(\alpha \wedge \beta, \alpha)}^{\llbracket \alpha \wedge \beta \rrbracket}},
$$

which would imply

$$
\left.\left.\left.\mathcal{O} b_{Y}^{\llbracket \alpha \rrbracket}\right|_{Y_{(\alpha, \beta)}^{\llbracket \alpha \rrbracket}} \cong \mathcal{O} b_{Y}^{\llbracket \alpha \wedge \beta \rrbracket}\right|_{Y_{(\alpha, \beta)}^{\llbracket \alpha \rrbracket}=Y_{(\beta, \alpha)}^{\llbracket \beta \rrbracket}} \cong \mathcal{O} b_{Y}^{\llbracket \beta \rrbracket}\right|_{Y_{(\beta, \alpha)}^{\llbracket \beta \rrbracket}} .
$$

For the case $\alpha \geq \beta$, by induction we only need to consider the case where

$$
\alpha=\left(\alpha_{1}, \cdots, \alpha_{k}\right) \geq \beta=\left(\beta_{1}, \cdots, \beta_{k+1}\right) .
$$

For simplicity we assume $\alpha_{i}=\beta_{i}$ for $i<k$ and $\alpha_{k}=\beta_{k} \cup \beta_{k+1}$. 
Now let $\left(\mathcal{W}_{i}, \varphi_{i}\right)_{1 \leq i \leq k}$, with $\mathcal{W}_{i} \subset Y \times_{T} Y_{(\alpha, \beta)}^{\llbracket \alpha \rrbracket}$ be the universal family of $Y \llbracket \alpha \rrbracket$ over $Y_{(\alpha, \beta)}^{\llbracket \alpha \rrbracket}$; let $\left(\tilde{\mathcal{W}}_{i}, \tilde{\varphi}_{i}\right)_{i=1}^{k+1}$ be the universal family of $Y^{\llbracket \beta \rrbracket}$ over $Y_{(\beta, \alpha)}^{\llbracket \beta \rrbracket}$. Because $\alpha_{i}=\beta_{i}$ for $i<k, \mathcal{W}_{i} \cong \tilde{\mathcal{W}}_{i}$ after identifying $Y_{(\alpha, \beta)}^{\llbracket \alpha \rrbracket} \cong Y_{(\beta, \alpha)}^{\llbracket \beta \rrbracket}$. On the other hand, by Corollary 1.3 the supports of $\tilde{\mathcal{W}}_{k}$ and $\tilde{\mathcal{W}}_{k+1}$ are disjoint closed subsets of $Y \times_{T} Y_{(\beta, \alpha)}^{\llbracket \beta \rrbracket}$; and their union form a flat family of zero-subschemes satisfying $\tilde{\mathcal{W}}_{k} \cup \tilde{\mathcal{W}}_{k+1}=\mathcal{W}_{k}$. As for the obstruction sheaves $\mathcal{O} b_{Y}^{\llbracket \alpha \rrbracket}$ and $\mathcal{O} b_{Y}^{\llbracket \beta \rrbracket}$, by definition,

and

$$
\begin{gathered}
\left.\mathcal{O} b_{Y}^{\llbracket \alpha \rrbracket}\right|_{Y_{(\alpha, \beta)}^{\llbracket \alpha \rrbracket}}=\bigoplus_{i=1}^{k} \mathcal{E} x t_{\mathrm{pr}_{2}}^{2}\left(\mathcal{I}_{\mathcal{W}_{i}}, \mathcal{I}_{\mathcal{W}_{i}}\right)_{0} \\
\left.\mathcal{O} b_{Y}^{\llbracket \beta \rrbracket}\right|_{Y_{(\beta, \alpha)}^{\llbracket \beta \rrbracket}}=\bigoplus_{i=1}^{k+1} \mathcal{E} x t_{\mathrm{pr}_{2}}^{2}\left(\mathcal{I}_{\tilde{\mathcal{W}}_{i}}, \mathcal{I}_{\tilde{\mathcal{W}}_{i}}\right)_{0} .
\end{gathered}
$$

Hence to prove the lemma we only need to check that

$$
\mathcal{E} x t_{\mathrm{pr}_{2}}^{2}\left(\mathcal{I}_{\mathcal{W}_{k}}, \mathcal{I}_{\mathcal{W}_{k}}\right)_{0} \cong \mathcal{E} x t_{\mathrm{pr}_{2}}^{2}\left(\mathcal{I}_{\tilde{\mathcal{W}}_{k}}, \mathcal{I}_{\tilde{\mathcal{W}}_{k}}\right)_{0} \oplus \mathcal{E} x t_{\mathrm{pr}_{2}}^{2}\left(\mathcal{I}_{\tilde{\mathcal{W}}_{k+1}}, \mathcal{I}_{\tilde{\mathcal{W}}_{k+1}}\right)_{0}
$$

But this is exactly what was proved in the last subsection.

\subsection{Obstruction sheaves under smooth isomorphism}

We next move to the obstruction sheaves of various moduli spaces of interests. We continue to denote by $X$ a smooth complex threefold.

Let $\mathcal{I}_{\mathcal{Z}_{i}}$ be the the ideal sheaves of $\mathcal{Z}_{1}, \cdots, \mathcal{Z}_{k} \subset \mathcal{U}_{0}^{\llbracket \alpha \rrbracket} \times_{X} \mathcal{U}$, which are part of the universal family of $X^{\llbracket \alpha \rrbracket}$; let $\pi_{1}$ be the first projection of $X^{\llbracket \alpha \rrbracket} \times X$. Then the obstruction sheaf of $X^{\llbracket \alpha \rrbracket}$ is

$$
\mathcal{O} b_{X}^{\llbracket \alpha \rrbracket}=\bigoplus_{i=1}^{k} \mathcal{E} x t_{\pi_{1}}^{2}\left(\mathcal{I}_{\mathcal{Z}_{i}}, \mathcal{I}_{\mathcal{Z}_{i}}\right)_{0} \cong \bigoplus_{i=1}^{k} \pi_{1 *} \mathcal{E} x t^{2}\left(\mathcal{O}_{\mathcal{Z}_{i}}, \mathcal{I}_{\mathcal{Z}_{i}}\right)
$$

Similarly, the obstruction sheaf of $\mathcal{V}_{0}^{\llbracket \alpha \rrbracket}$ is defined to be the relative extension sheaf

$$
\mathcal{O} b_{\mathcal{V}_{0}}^{\llbracket \alpha \rrbracket}=\bigoplus_{i=1}^{k} \mathcal{E} x t_{\pi_{1}}^{2}\left(\mathcal{I}_{\mathcal{W}_{i}}, \mathcal{I}_{\mathcal{W}_{i}}\right)_{0} \cong \bigoplus_{i=1}^{k} \pi_{1 *} \mathcal{E} x t^{2}\left(\mathcal{O}_{\mathcal{W}_{i}}, \mathcal{I}_{\mathcal{W}_{i}}\right)
$$

in which $\mathcal{I}_{\mathcal{W}_{i}}$ are ideal sheaves of the subschemes $\mathcal{W}_{1} \cdots, \mathcal{W}_{k} \subset \mathcal{V}_{0}^{\llbracket \alpha \rrbracket} \times_{X} \mathcal{V}$ that are part of the universal family of $\mathcal{V}_{0}^{\llbracket \alpha \rrbracket}$; that $\pi_{1}$ is the first projection of $\mathcal{V}_{0}^{\llbracket \alpha \rrbracket} \times_{X} \mathcal{V}$. 
These obstruction sheaves are related in the obvious way. First, we let

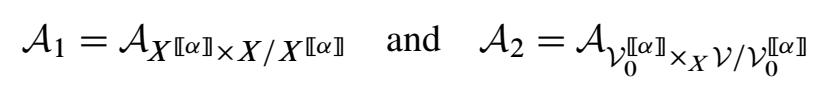

respectively be the sheaf of smooth functions on $X^{\llbracket \alpha \rrbracket} \times X$ and $\mathcal{V}_{0}^{\llbracket \alpha \rrbracket} \times_{X} \mathcal{V}$ that are analytic along fibers of $X^{\llbracket \alpha \rrbracket} \times X / X^{\llbracket \alpha \rrbracket}$ and $\mathcal{V}_{0}^{\llbracket \alpha \rrbracket} \times_{X} \mathcal{V} / \mathcal{V}_{0}^{\llbracket \alpha \rrbracket}$. Because $\Psi_{\alpha}$ of Lemma 2.6 is induced by the universal family and because the tautological map

$$
h=\left(\Psi_{\alpha}, p_{2} \circ \psi\right): \mathcal{V}_{0}^{\llbracket \alpha \rrbracket} \times_{X} \mathcal{V} \longrightarrow X^{\llbracket \alpha \rrbracket} \times X,
$$

where $p_{2} \circ \psi: \mathcal{V} \rightarrow X$ is the composite of $\psi: \mathcal{V} \rightarrow X \times X$ with the second projection of $X \times X$, maps fibers to fibers and is analytic along fibers,

$$
h^{*}\left(\mathcal{I}_{\mathcal{Z}_{i}} \otimes \mathcal{A}_{1}\right) \cong \mathcal{I}_{\mathcal{W}_{i}} \otimes \mathcal{A}_{2}
$$

Here the tensor products are over the sheaves $\mathcal{O}_{X \llbracket \alpha \rrbracket_{X X}}$ and $\mathcal{O}_{\mathcal{V}_{0}^{\llbracket \alpha \rrbracket}}{\mathbb{X _ { X }}}_{\mathcal{V}}$. On the other hand, by the property of relative extension sheaf, the tensor product

$$
\begin{aligned}
\mathcal{O} b_{\mathcal{V}_{0}}^{\llbracket \alpha \rrbracket} \otimes \mathcal{A}_{\mathcal{V}_{0}^{\llbracket \alpha \rrbracket}} & \cong \bigoplus_{i=1}^{k} \pi_{1 *}\left(\mathcal{E x t}_{\mathcal{A}_{2}}^{3}\left(\mathcal{I}_{\mathcal{W}_{i}}, \mathcal{I}_{\mathcal{W}_{i}}\right) \otimes \mathcal{A}_{2}\right) \\
& \cong \bigoplus_{i=1}^{k} \pi_{1 *} \mathcal{E} x t_{\mathcal{A}_{2}}^{3}\left(\mathcal{I}_{\mathcal{W}_{i}} \otimes \mathcal{A}_{2}, \mathcal{I}_{\mathcal{W}_{i}} \otimes \mathcal{A}_{2}\right)
\end{aligned}
$$

Here the extension sheaf $\mathcal{E x t}_{\mathcal{A}_{2}}^{3}(\cdot, \cdot)$ is defined using locally free resolution of locally free sheaves of $\mathcal{A}_{2}$-modules.

Because of the isomorphism (20), the last term in the above isomorphisms is canonically isomorphic to

$$
\begin{aligned}
& \cong \bigoplus_{i=1}^{k} \pi_{1 *}\left(\mathcal{E}_{\mathcal{A}_{2}}^{3}\left(h^{*}\left(\mathcal{I}_{\mathcal{Z}_{i}} \otimes \mathcal{A}_{1}\right), h^{*}\left(\mathcal{I}_{\mathcal{Z}_{i}} \otimes \mathcal{A}_{1}\right)\right)\right) \\
& \cong \bigoplus_{i=1}^{k} \pi_{1 *}\left(\mathcal{E} x t_{\mathcal{A}_{1}}^{3}\left(\mathcal{O}_{\mathcal{Z}_{i}} \otimes \mathcal{A}_{1}, \mathcal{I}_{\mathcal{Z}_{i}} \otimes \mathcal{A}_{1}\right) \cong \Psi_{\alpha}^{*}\left(\mathcal{O} b_{X}^{\llbracket \alpha \rrbracket} \otimes \mathcal{A}_{X} \llbracket \alpha\right)\right.
\end{aligned}
$$

This proves that

$$
\Psi_{\alpha}^{*}\left(\mathcal{O} b_{X}^{\llbracket \alpha \rrbracket} \otimes \mathcal{A}_{X} \llbracket \alpha \rrbracket\right) \cong \mathcal{O} b_{\mathcal{V}_{0}}^{\llbracket \alpha \rrbracket} \otimes \mathcal{A}_{\mathcal{V}_{0}^{\llbracket \alpha \rrbracket}}
$$




\section{Representing Virtual Cycles}

We will begin this section with a quick review of the construction of the virtual cycle of a scheme with perfect obstruction theory. For more details of this construction, please consult Behrend and Fantechi [4] or Li and Tian [7].

\subsection{Virtual cycles via Gysin map}

For the moment, we assume $W$ is a quasi-project scheme with a perfect obstruction theory and obstruction sheaf $\mathcal{O} b$. Following [4; 7], the virtual cycle of $W$ is constructed via

(1) finding a vector bundle $V$ on $W$ and a surjective sheaf homomorphism ${ }^{3} \mathcal{V} \rightarrow$ $\mathcal{O} b$; then the obstruction theory of $W$ provides us a unique cone cycle ${ }^{4} C \subset V$ of codimension $\mathrm{rk} V$;

(2) defining the virtual cycle $[W]^{\mathrm{vir}}=0_{V}^{*}[C]$ via the Gysin homomorphism

$$
0_{V}^{*}: H_{*}^{B M}(V, \mathbb{Z}) \rightarrow H_{*}(W, \mathbb{Z}) .
$$

Since every subvariety of $V$ defines a class in the Borel-Moore homology group, $0_{V}^{*}[C]$ is well-defined.

Before we move on, a few comments are in order.

Usually, the Gysin map is defined as a homomorphism between Chow groups. For us, we shall use the Borel-Moore homology group and use intersecting with smooth sections to define this homomorphism.

The cone cycle $C \subset V$ is unique in the following sense. To each closed $w \in W$, we let $\widehat{w}$ be the formal completion of $W$ along $w$ and fix an embedding

$$
\widehat{w} \subset T \triangleq \widehat{S} \operatorname{Spec} \mathbf{k} \llbracket T_{w} W^{\vee} \rrbracket
$$

that is consistent with the tangent space at their only closed points. We let $O=$ $\mathcal{O} b \otimes_{\mathcal{O}_{W}} \mathbf{k}(w)$ be the obstruction space to deforming $w$ in $W$. Then the Kuranishi map of the obstruction theory provides a canonical embedding of the normal cone $C_{\widehat{w}} \widehat{T}$ to $\widehat{w}$ in $\widehat{T}$ :

$$
C_{\widehat{w}} \widehat{T} \subset \widehat{w} \times O
$$

\footnotetext{
${ }^{3}$ In this paper, whenever we use a Roman alphabet, say $V$, to denote a vector bundle, we will use its counter part $\mathcal{V}$ to denote the sheaf of regular sections $\mathcal{O}_{W}(V)$.

${ }^{4} \mathrm{~A}$ cycle is a finite union $\sum m_{i} D_{i}$ of subvarieties $D_{i}$ with integer coefficients $m_{i}$; it is a cone cycle if all $D_{i}$ are cones in $V$.
} 
where the later is viewed as a vector bundle over $\widehat{w}$. The uniqueness of $C$ asserts that there is a vector bundle homomorphism

$$
\eta_{w}: V \times_{W} \widehat{w} \longrightarrow O \times \widehat{w}
$$

extending the homomorphism $\left.V\right|_{w} \rightarrow O$ induced by $\mathcal{V} \rightarrow \mathcal{O} b$ such that

$$
\eta^{*} C_{\widehat{w}} \widehat{T}=C \cap\left(V \times_{W} \widehat{w}\right),
$$

Lastly, the resulting cycle $0_{V}^{*}[C] \in A_{*} W$ is independent of the choice of $\mathcal{V} \rightarrow \mathcal{O} b$.

As we will see in the later part of this paper, it will be useful to eliminate the dependence of constructing $[W]^{\text {vir }}$ on the choice of $\mathcal{V} \rightarrow \mathcal{O} b$. To achieve this, we will use smooth sections of $\mathcal{O} b$ to define the cycle $[W]^{\text {vir }}$.

Before we do that, we shall first recall the notion of pseudo-cycles.

\subsection{Pseudo-Cycles}

In this work, we shall use pseudo-cycles to represent homology classes in a stratifiable space.

Let $\Theta \subset W$ be a triangulable closed subset of a stratified space $W$. We shall fix a Riemannian metric ${ }^{5}$ on $W$ and denote by $\Theta_{\epsilon}$ the $\epsilon$-tubular neighborhood $\Theta$ in $W$.

Definition 4.1 A $(\Theta, \epsilon)$-relative $d$-dimensional pseudo-chain is a pair $(f, \Sigma)$ of a smooth, oriented $d$-dimensional manifold with smooth boundary $\partial \Sigma$ and a continuous map $f: \Sigma \rightarrow W$ such that $f$ is smooth over $\Sigma-f^{-1}\left(\Theta_{\epsilon}\right)$.

We denote the $\mathbb{Z}$-linear span of all such pseudo-chains by $P C h_{d}(W)_{\Theta}$ with $\epsilon-$ implicitly understood.

We define an equivalence relation $\cong$ on $P C h_{d}(W)_{\Theta}$ as follows. For notation brevity, we shall use $[f]_{p c}$ to denote the pseudo-cycle $(f, \Sigma)$ with $\Sigma$ implicitly understood. We call two such chains $\left[f_{1}\right]_{p c}$ and $\left[f_{1}\right]_{p c}$ equivalent if there are open subsets $A_{i} \subset \Sigma_{i}$ and orientation preserving diffeomorphism $\phi: A_{1} \rightarrow A_{2}$ so that $\Sigma_{i}-A_{i} \subset f_{i}^{-1}\left(\Theta_{\epsilon}\right)$ for $i=1$ and 2 , and $\left.f_{2} \circ \phi\right|_{A_{1}}=\left.f_{1}\right|_{A_{1}}$; we define $\left[f_{1} \cup f_{2}\right]_{p c} \cong\left[f_{1}\right]_{p c}+\left[f_{2}\right]_{p c}$ with the union $\left[f_{1} \cup f_{2}\right]_{p c}$ be the map $\Sigma_{1} \cup \Sigma_{2} \rightarrow W$ induced by $f_{1}$ and $f_{2}$; we define $[-f]_{p c} \cong-[f]_{p c}$ with $[-f]_{p c}$ be $f: \Sigma^{-} \rightarrow W$ and $\Sigma^{-}$the space $\Sigma$ with the opposite orientation. The equivalence relation $\cong$ generates an ideal in the Abelian group $P C h_{d}(W)_{\Theta}$.

\footnotetext{
${ }^{5}$ We can embed $W$ in a smooth space and use the induced Riemannian metric on the ambient space.
} 
There is an obvious boundary homomorphism

$$
\text { д: } P C h_{d}(W)_{\Theta} \longrightarrow P C h_{d-1}(W)_{\Theta}
$$

that sends any $[f]_{p c}$ to $[\partial f]_{p c}$ with $\partial f$ is the restriction of $f$ to $\partial \Sigma$. The kernel of its induced homomorphism is defined to be the space of pseudo-cycles relative to $\Theta$ :

$$
P C y_{d}(E)_{\Theta} \triangleq \operatorname{ker}\left\{\partial: P C h_{d}(W)_{\Theta} \longrightarrow P C h_{d-1}(W)_{\Theta} / \cong\right\} .
$$

The proof of the following lemma is standard.

Lemma 4.2 Suppose $\operatorname{dim}_{\mathbb{R}} \Theta \leq d-2$ and suppose the $\epsilon-$ tubular neighborhood $\Theta_{\epsilon}$ is a deformation retract to $\Theta$. Then every $(\Theta, \epsilon)$-relative pseudo-cycle $[f]_{p c} \in P c y_{d}(W)_{\Theta}$ defines canonically a homology class $[f] \in H_{d}(W, \mathbb{Z})$.

One version of pseudo-cycle we shall repeatedly use is the following:

Remark 4.3 Any pair $(B, \Theta)$ of a closed subset $B \subset W$ and a stratifiable closed $\Theta \subset W$ such that $B-\Theta$ is a smooth, oriented $d$-dimensional manifold and $\operatorname{dim}_{\mathbb{R}} \Theta \leq$ $d-2$ is a $(\Theta, \epsilon)$-relative pseudo-cycle for all $\epsilon>0$.

This can be seen as follows. For any $\epsilon>0$, we pick an open $O \subset B \cap \Theta_{\epsilon / 2}$ so that $\Sigma=B-O$ is a smooth manifold with smooth boundary. Then the identity map $f: \Sigma \rightarrow B \subset W$ is a $(\Theta, \epsilon)$-relative pseudo-cycle.

In case $W$ is stratifiable, which is the case when $W$ is an open subset of a quasiprojective scheme, $\Theta_{\epsilon}$ deformation retract to $\Theta$ for all sufficiently small $\epsilon$. Because $\operatorname{dim}_{\mathbb{R}} \Theta \leq d-2$, for all sufficiently small $\epsilon$ these $(\Theta, \epsilon)$-relative pseudo cycles all represent the same homology class in $H_{d}(W, \mathbb{Z})$. Because of this, in the future we will call $B$ a $\Theta$-relative pseudo-cycle and will denote by $[B] \in H_{d}(W, \mathbb{Z})$ the resulting homology class. In case $\Theta$ is the singular locus of $B$, we shall call $B$ a pseudo-cycle directly with $\Theta=B_{\text {sing }}$ implicitly understood.

\subsection{Cycle representatives via smooth sections of sheaves}

We now investigate how to intersect with smooth sections to define the Gysin homomorphism of a cone cycle in a vector bundle over $W$.

Let $C$ be a pure $\mathbb{C}$-dimension $r$ algebraic cone cycle in a vector bundle $\pi: V \rightarrow W$;

$$
C=\sum m_{i} C_{i}
$$

its irreducible components decomposition. We pick a stratification $\mathcal{S}$ of $W$ and a stratification $\mathcal{S}^{\prime}$ of $\cup_{i} C_{i}$ so that the induced map $\cup_{i} C_{i} \rightarrow W$ is a stratified map. Also, 
to each stratum $S^{\prime} \in \mathcal{S}^{\prime}$ with $S=\pi\left(S^{\prime}\right)$ its image stratum, we shall denote by $V_{S}$ the restriction to $S$ of $V$; for a smooth section $s \in \mathcal{A}_{\mathcal{S}}(V)$, we denote its graph by $\Gamma_{s} \subset V$.

Definition 4.4 A smooth section $s \in \mathcal{A}_{\mathcal{S}}(V)$ is said to intersect transversally with $C$ if every stratum $S^{\prime} \subset \cup_{i} C_{i}$, which is a smooth subset in $V_{S}$, intersects transversally with $\Gamma_{S} \cap V_{S}$ inside $V_{S}$.

By embedding $W$ in a projective space and extending $V$, we can apply the standard Sard's transversality theorem to conclude that the set of smooth sections that intersect transversally with $C$ is dense in the space of all smooth sections.

Let $s$ be a section that intersects transversally with $C$. We claim that the intersection $\Gamma_{s} \cap C$ is a pseudo-cycle. Indeed, let $S_{i} \subset C_{i}$ be the open stratum of $C_{i}$; then $S_{i} \subset C_{i}$ is smooth, open and Zariski dense; therefore, $\operatorname{dim}_{\mathbb{R}} C_{i}-S_{i} \leq \operatorname{dim}_{\mathbb{R}} C_{i}-2$. Suppose $\operatorname{dim} C_{i}=d$ (for all $i$ ) and $\mathrm{rk} V=r$. Then $\Gamma_{s} \cap S_{i}$ is a smooth, oriented manifold of real dimension $2 d-2 r$; its complement $\Gamma_{s} \cap\left(C_{i}-S_{i}\right)$ has real dimension at most $2 d-2 r-2$. According to Remark 4.3, by taking

$$
\Theta=\cup_{i} \Gamma_{s} \cap\left(C_{i}-S_{i}\right),
$$

the set $\Gamma_{s} \cap C_{i}$ becomes a $2 d-2 r$-dimensional pseudo-cycle (relative to $\Theta$ ). We denote this pseudo-cycle by $\left(\Gamma_{s} \cap C_{i}\right)_{p c}$ and denote its image pseudo-cycle under the projection $\pi: V \rightarrow W$ by $\pi_{*}\left(\Gamma_{s} \cap C_{i}\right)_{p c}$. Finally, by linearity,

$$
\left(\Gamma_{s} \cap C\right)_{p c}=\sum_{i}\left(\Gamma_{s} \cap C_{i}\right)_{p c} \quad \text { and } \quad \pi_{*}\left(\Gamma_{s} \cap C\right)_{p c}=\sum_{i} \pi_{*}\left(\Gamma_{s} \cap C_{i}\right)_{p c} .
$$

It is immediate to check that its associated homology class given by Lemma 4.2 is the Gysin homomorphism image of $[C]$

$$
0_{V}^{*}[C]=\left[\pi_{*}\left(\Gamma_{S} \cap C\right)_{p c}\right] \in H_{2 d-2 r}(W, \mathbb{Z}) .
$$

We now apply this technique to construct the virtual cycle $[W]^{\text {vir }}$, assuming that $W$ is quasi-projective with a perfect obstruction theory and the obstruction sheaf $\mathcal{O} b$. As we mentioned, we first pick a locally free sheaf $\mathcal{V}$ and a quotient sheaf homomorphism $\mathcal{V} \rightarrow \mathcal{O} b$, thus obtaining a cone $C \subset V$ given by the obstruction theory of $W$. We then pick the standard pair of stratification $\mathcal{S}^{\prime}$ of $C$ and $\mathcal{S}$ of $W$ that respects the morphism $C \rightarrow W$ and the loci of non-locally freeness of $\mathcal{O} b$. Then as was argued, we can find a section $s \in \mathcal{A}_{\mathcal{S}}(V)$ that intersects transversally with $C$. The virtual fundamental cycle $[W]^{\mathrm{vir}}$, which is $0_{V}^{*}[C]$, is the homology class given by the pseudo-cycle

$$
[W]^{\mathrm{vir}}=0_{V}^{*}[C]=\left[\pi_{*}\left(C \cap \Gamma_{s}\right)_{p c}\right] \in H_{*}(W, \mathbb{Z}) .
$$


We next show that such pseudo-cycle can be constructed using the image section $\xi \in \mathcal{A}_{\mathcal{S}}(\mathcal{O} b)$ of $s$ under the homomorphism $\mathcal{A}_{\mathcal{S}}(V) \rightarrow \mathcal{A}_{\mathcal{S}}(\mathcal{O} b)$. To this end, we need first to recover the pseudo-cycle $\pi_{*}\left(\Gamma_{S} \cap C\right)_{p c}$ using the smooth section $\xi \in \mathcal{A}_{\mathcal{S}}(\mathcal{O} b)$. Suppose $\mathcal{V}^{\prime} \rightarrow \mathcal{O} b$ is a surjective homomorphism and $t \in \mathcal{A}_{\mathcal{S}}\left(V^{\prime}\right)$ is a lift of $\xi$ under $\mathcal{A}_{\mathcal{S}}\left(\mathcal{V}^{\prime}\right) \rightarrow \mathcal{A}_{\mathcal{S}}(\mathcal{O} b)$, which exists by the exact sequence at the end of subsection 2.2. We claim that $t$ intersects transversally with the virtual normal cone $C^{\prime} \subset V^{\prime}$ and

$$
\pi_{*}\left(\Gamma_{s} \cap C\right)_{p c}=\pi_{*}^{\prime}\left(\Gamma_{t} \cap C^{\prime}\right)_{p c}
$$

as pseudo-cycles in $W$.

We first prove the case where $\mathcal{V}^{\prime}=\mathcal{V}$ and $t \in \mathcal{A}_{\mathcal{S}}(V)$ is another lifting of $\xi$. We first show that as sets $\pi\left(\Gamma_{s} \cap C\right)=\pi^{\prime}\left(\Gamma_{t} \cap C^{\prime}\right)$. For this, we consider the section $s-t \in \mathcal{A}_{\mathcal{S}}(V)$ and its induced fiberwise translation

$$
\ell_{s-t}: V \rightarrow V ; \quad x \in V_{w} \longmapsto x+s(w)-t(w) \in V_{w} .
$$

Because of [7], the fiber $C \cap V_{w}$ of the cone over $w$ is translation invariant under vectors in

$$
K_{w}=\operatorname{ker}\left\{\left.V_{w} \rightarrow \mathcal{O} b\right|_{w}\right\} .
$$

Therefore $\ell_{s-t}: V \rightarrow V$ maps $C$ to $C$. But on the other hand, $\ell_{s-t}$ maps $\Gamma_{t}$ to $\Gamma_{s}$, hence $\ell_{s-t}\left(\Gamma_{t} \cap C\right)=\Gamma_{s} \cap C$, which proves (23).

It remains to show that $t$ intersects $C$ transversally and the induced orientation on $\pi\left(\Gamma_{S} \cap C\right)$ coincides with that of $\pi\left(\Gamma_{t} \cap C\right)$. First, because the stratification $\mathcal{S}$ respects the non-locally freeness of $\mathcal{O} b$, the restriction $\mathcal{O} b \otimes_{\mathcal{O}_{W}} \mathcal{O}_{S}$ is locally free. Hence the collection $\left\{K_{w} \mid w \in S\right\}$ forms a subbundle of $V_{S}$. By the translation invariance of $C \cap V_{w}$ under $K_{w}$ and by the minimality of the stratification $\mathcal{S}^{\prime}, S^{\prime} \cap V_{w}$ is invariant under the translations by vectors in $K_{w}$; hence $S^{\prime}=\ell_{s-t}\left(S^{\prime}\right)$.

On the other hand, since the map $\ell_{s-t}: V_{S} \rightarrow V_{S}$ is a smooth diffeomorphism, $\Gamma_{t}$ intersects transversally with $S^{\prime}$ if and only if its image $\ell_{s-t}\left(\Gamma_{t}\right)=\Gamma_{s}$ intersects transversally with $\ell_{s-t}\left(S^{\prime}\right)=S^{\prime}$, which is true by our choice of $s$. Hence $\Gamma_{t}$ intersects transversally with $S^{\prime}$. In particular, $\ell_{s-t}\left(\Gamma_{t} \cap C\right)=\Gamma_{s} \cap C$ and hence $\pi_{*}\left(\Gamma_{t} \cap C\right)_{p c}=$ $\pi_{*}\left(\Gamma_{s} \cap C\right)_{p c}$.

Next we consider the general situation where $\mathcal{V}^{\prime} \rightarrow \mathcal{O} b$ is an arbitrary quotient sheaf homomorphism by a locally free sheaf. We claim that by picking a lifting $t \in \mathcal{A}_{\mathcal{S}}\left(\mathcal{V}^{\prime}\right)$ of $\xi \in \mathcal{A}_{\mathcal{S}}(\mathcal{O} b)$, we obtain the identical cycle representatives of $[W]^{\mathrm{vir}}$. Indeed, by our previous discussion, we only need to consider the case that $\mathcal{V}$ is a quotient sheaf of $\mathcal{V}^{\prime}$ and $\mathcal{V}^{\prime} \rightarrow \mathcal{O} b$ is the composite of

$$
\mathcal{V}^{\prime} \longrightarrow \mathcal{V} \longrightarrow \mathcal{O} b .
$$


In this case, the cone cycle $C^{\prime} \subset V^{\prime}$ is merely the pull back of $C \subset V$ via the induced vector bundle homomorphism $\varphi: V^{\prime} \rightarrow V$. Now let $t^{\prime} \in \mathcal{A}_{\mathcal{S}}\left(\mathcal{V}^{\prime}\right)$ be a lifting of $s \in \mathcal{A}_{\mathcal{S}}(\mathcal{V})$, and let $\pi^{\prime}: V^{\prime} \rightarrow W$ be the projection. Then because $C^{\prime}=\varphi^{*} C$,

$$
\pi_{*}^{\prime}\left(\Gamma_{t} \cap \varphi^{*} C\right)_{p c}=\pi_{*}^{\prime}\left(\Gamma_{t^{\prime}} \cap \varphi^{*} C\right)_{p c}=\pi_{*}\left(\Gamma_{s} \cap C\right)_{p c}
$$

as pseudo cycles. This shows that the pseudo-cycle representative $\pi\left(\Gamma_{S} \cap C\right)$ of $[W]^{\mathrm{vir}}$ only depends on the section $\xi \in \mathcal{A}_{\mathcal{S}}(\mathcal{O} b)$.

This way, those sections $\xi \in \mathcal{A}_{\mathcal{S}}(\mathcal{O} b)$ whose lifts intersect transversally with the normal cone provide us pseudo-cycle representatives of the virtual cycle $[W]^{\text {vir }}$. In the following, we shall denote such representative by $D(\xi)_{p c}$.

In the remainder of this paper, for a scheme $W$ with obstruction sheaf $\mathcal{O} b$, we shall fix a locally free sheaf $\mathcal{O}_{W}(V)$ that surjects onto $\mathcal{O} b$ with $C$ the virtual normal cone in $V$ of the obstruction theory of $W$. We say that a smooth section $\xi \in \mathcal{A}_{\mathcal{S}}(\mathcal{O} b)$ is a good section if it has a lift $s \in \mathcal{A}_{\mathcal{S}}(V)$ that intersects transversally with $C$. By the previous construction, the image $\pi_{*}\left(\Gamma_{s} \cap C\right)_{p c}$ defines a closed pseudo-cycle $D(\xi)_{p c}$.

We summarize this subsection in the following Proposition.

Proposition 4.5 Let the notation be as before. Then any good section $\xi \in \mathcal{A}_{\mathcal{S}}(\mathcal{O} b)$ defines a pseudo-cycle $D(\xi)$ in $W$ whose associated homology class is the virtual cycle $[W]^{\text {vir }}$ in $H_{*}(W, \mathbb{Z})$.

\subsection{Virtual cycle of Hilbert schemes of $\alpha$-points}

We shall employ smooth sections to construct cycle representatives of the virtual cycles of $Y^{\llbracket \alpha \rrbracket}$ for a smooth family of quasi-projective threefolds $Y / T$ over a smooth base $T$. But before we do that, we shall first define how cycles are pulled back by the tautological map $Y^{\llbracket \alpha \rrbracket} \rightarrow Y^{[\alpha]}$.

We begin with defining the $\alpha$-multiplicity of the symmetrization morphism

$$
S_{\alpha}: Y^{\Lambda} \longrightarrow Y^{(\alpha)}
$$

Let $x \in Y^{\Lambda}$ be any element. We define the multiplicity $m_{\alpha}(x)$ be the number of permutations of $\Lambda$ that fix $x$ and leave $\alpha$ invariant. Namely,

$$
m_{\alpha}(x)=\#\{\sigma \in \operatorname{Symm}(\Lambda) \mid \sigma(\alpha)=\alpha, \sigma(x)=x\} .
$$


It is easy to see that all elements in $\left(S_{\alpha}\right)^{-1} S_{\alpha}(x)$ have identical $\alpha$-multiplicities; their summations satisfies ${ }^{6}$

$$
\sum_{y \in\left(\mathcal{S}^{\alpha}\right)^{-1} \mathcal{S}^{\alpha}(x)} m_{\alpha}(y)=\alpha !
$$

For any $z \in Y^{\llbracket \alpha \rrbracket}$ lies over $x \in Y^{\Lambda}$, we define its $\alpha$-multiplicity $m_{\alpha}(z)=m_{\alpha}(x)$.

Because the virtual normal cone is constructed based on the obstruction theory of the Hilbert scheme of points $I_{Y / T}(0, n)$, we need to work with the tautological morphism

$$
\varphi_{\alpha}: Y^{\llbracket \alpha \rrbracket} \longrightarrow I_{Y / T}(0, \alpha)
$$

with $\quad I_{Y / T}(0, \alpha)=I_{Y / T}\left(0, m_{1}\right) \times_{T} \cdots \times_{T} I_{Y / T}\left(0, m_{k}\right), \quad$ where $m_{i}=\left|\alpha_{i}\right|$.

We let $F$ be a vector bundle on $I_{Y / T}(0, \alpha)$ and let $C \subset F$ be a cycle, which is a linear combination of subvarieties of $F$. We let $E$ be the pull back vector bundle $\varphi_{\alpha}^{*} F$ on $Y^{\llbracket \alpha \rrbracket}$; let $\phi_{\alpha}: E \rightarrow F$ and $\pi_{\alpha}: E \rightarrow Y^{\llbracket \alpha \rrbracket}$ be the obvious projections.

Definition 4.6 For any subvariety $D \subset E$ we define the $\alpha$-multiplicity $m_{\alpha}(D)$ of $D$ be the $\alpha$-multiplicity of the general point of the image $\pi_{\alpha}(D) \subset Y \llbracket \alpha \rrbracket$. For any subvariety $C \subset F$ and irreducible decomposition $\phi_{\alpha}^{-1}(C)=\cup_{i=1}^{r} D_{i}$, we define the pull-back

$$
\phi_{\alpha}^{*} C=\sum_{i=1}^{r} m_{\alpha}\left(D_{i}\right) D_{i} .
$$

We define the pull back of any cycle by extension via linearity.

The identity (24) implies that for any cycle $C$ in $F$

$$
\phi_{\alpha *} \phi_{\alpha}^{*} C=\alpha ! C .
$$

The virtual cycle of $Y \llbracket \alpha \rrbracket$ will be defined as the Gysin map image of the pull back virtual normal cone given by the obstruction theory of $I_{Y / T}(0, \alpha)$. Let $\mathcal{F}_{\alpha}$ be a locally free sheaf on $I_{Y / T}(0, \alpha)$ that makes the obstruction sheaf of $I_{Y / T}(0, \alpha)$ its quotient sheaf. Then the obstruction theory of $I_{Y / T}(0, \alpha)$ provides us a cone cycle $C_{\alpha} \in A_{*} F_{\alpha}$ in the vector bundle $F_{\alpha}$ associated to $\mathcal{E}_{\alpha}$. We let $E_{\alpha}$ be the pull back vector bundle over $Y^{\llbracket \alpha \rrbracket}$; let $\phi_{\alpha}: E_{\alpha} \rightarrow F_{\alpha}$ be the projection, and let

$$
C_{\alpha}=\phi^{*} C_{\alpha}
$$

be the pull back cycle in $E_{\alpha}$ defined in Definition 4.6. Since the pull back of the obstruction sheaf of $I_{Y / T}(0, \alpha)$ is canonically isomorphic to the obstruction sheaf of

${ }^{6}$ We define $\alpha !=\alpha_{1} ! \cdots \alpha_{k} !$ 
$Y^{\llbracket \alpha \rrbracket}$, the sheaf $\mathcal{E}_{\alpha}=\mathcal{O}\left(E_{\alpha}\right)$ has the obstruction sheaf $\mathcal{O} b_{Y}^{\llbracket \alpha \rrbracket}$ of $Y^{\llbracket \alpha \rrbracket}$ as its quotient sheaf. By abuse of notation, we will call the cycle $C_{\alpha} \in A_{*} E_{\alpha}$ the virtual cone of the obstruction theory of $Y^{\llbracket \alpha \rrbracket}$.

Definition 4.7 We define the virtual fundamental class

$$
\left[Y^{\llbracket \alpha \rrbracket}\right]^{\mathrm{vir}}=0_{E_{\alpha}}^{*}\left(C_{\alpha}\right) \in H_{*}\left(Y^{\llbracket \alpha \rrbracket}, \mathbb{Z}\right) .
$$

Since the push-forward of $\left[Y^{\llbracket \alpha \rrbracket}\right]^{\text {vir }}$ under $Y \llbracket \alpha \rrbracket \rightarrow I_{Y / T}(0, \alpha)$ is $\alpha$ ! times the virtual fundamental cycle $\left[I_{Y / T}(0, \alpha)\right]^{\mathrm{vir}}$, it is independent of the choice of $E_{\alpha}$, thus is welldefined.

To get an explicit cycle representative, we can take a good section $\xi_{\alpha}$ of $E_{\alpha}$ that intersects the cone $C_{\alpha}$ transversally to form a pseudo-cycle

$$
D\left(\xi_{\alpha}\right)=\pi_{\alpha}\left(\Gamma_{\xi_{\alpha}} \cap C_{\alpha}\right)_{p c} \in P C y_{*}\left(Y^{\llbracket \alpha \rrbracket}\right) .
$$

As was shown before, the cycle $D\left(\xi_{\alpha}\right)$ only depend on the image section $s_{\alpha} \in$ $\mathcal{A}_{\mathcal{S}}\left(\mathcal{O} b_{Y}^{\llbracket \alpha \rrbracket}\right)$ of $\xi_{\alpha}$. Hence to eliminate the dependence on $E_{\alpha}$, we shall denote $D\left(\xi_{\alpha}\right)$ by $D\left(s_{\alpha}\right)$. We have

$$
\left[D\left(s_{\alpha}\right)\right]=\left[Y^{\llbracket \alpha \rrbracket}\right]^{\mathrm{vir}} \in H_{*}\left(Y^{\llbracket \alpha \rrbracket}, \mathbb{Z}\right) .
$$

\section{Approximation of Virtual cycles}

Because the partial equivalence $Y_{(\alpha, \beta)}^{\llbracket \alpha \rrbracket} \cong Y_{(\beta, \alpha)}^{\llbracket \beta \rrbracket}$ is functorial, we expect that the obstruction sheaves, the virtual normal cones, and the cycle representatives of the virtual cycles of $Y \llbracket \alpha \rrbracket$ and $Y \llbracket \beta \rrbracket$ are identical over $Y_{(\alpha, \beta)}^{\llbracket \alpha \rrbracket} \cong Y_{(\beta, \alpha)}^{\llbracket \beta \rrbracket}$. It is the purpose of this section to show that this is the case.

\subsection{Cones under equivalence}

Our immediate task is to compare the sheaves $\mathcal{O} b_{Y}^{\llbracket \alpha \rrbracket}$ with $\mathcal{O} b_{Y}^{\llbracket \beta \rrbracket}$ and compare their respective virtual normal cones. Since for different $\alpha$ and $\beta$, the mentioned partial equivalence follows from the equivalence $Y_{(\alpha, \alpha \wedge \beta)}^{\llbracket \alpha \rrbracket} \cong Y_{(\alpha \wedge \beta, \alpha)}^{\llbracket \alpha \wedge \beta \rrbracket}$ and $Y_{(\beta, \alpha \wedge \beta)}^{\llbracket \beta \rrbracket} \cong Y_{(\alpha \wedge \beta, \beta)}^{\llbracket \alpha \wedge \beta \rrbracket}$, for our purpose we only need to investigate the case where $\alpha>\beta$.

We first set up the notation. Let $\alpha>\beta$ be any pair. In this section we will fix once and for all an indexing

$$
\alpha=\left(\alpha_{1}, \cdots, \alpha_{k}\right) \quad \text { and } \beta=\left(\beta_{11}, \cdots, \beta_{1 l_{1}}, \cdots, \beta_{k 1}, \cdots \beta_{k l_{k}}\right)
$$

so that $\beta_{i 1} \cup \cdots \cup \beta_{i l_{i}}=\alpha_{i}$. Since $\alpha>\beta$, such indexing exists. 
We let $\widetilde{\mathcal{Z}}_{1}, \cdots, \widetilde{\mathcal{Z}}_{k}$ be subschemes of $Y_{(\alpha, \beta)}^{\llbracket \alpha \rrbracket} \times_{T} Y$ that are part of the universal family of $Y^{\llbracket \alpha \rrbracket}$ over $Y_{(\alpha, \beta)}^{\llbracket \alpha \rrbracket}$; we let $\tilde{\mathcal{W}}_{11}, \cdots, \widetilde{\mathcal{W}}_{k l_{k}}$ be subschemes of $Y_{(\beta, \alpha)}^{\llbracket \beta \rrbracket} \times_{T} Y$ that are part of the universal family of $Y^{\llbracket \beta \rrbracket}$ over $Y_{(\beta, \alpha)}^{\llbracket \beta \rrbracket}$.

The partial equivalence $Y_{(\alpha, \beta)}^{\llbracket \alpha \rrbracket} \cong Y_{(\beta, \alpha)}^{\llbracket \beta \rrbracket}$ induces a rational map from $I_{Y / T}(0, \beta)$ to $I_{Y / T}(0, \alpha)$. Let

$$
\varrho_{\alpha}: Y_{(\alpha, \beta)}^{\llbracket \alpha \rrbracket} \longrightarrow I_{Y / T}(0, \alpha)
$$

be the tautological morphism that is induced by the families $\left(\mathcal{Z}_{1}, \cdots, \mathcal{Z}_{k}\right)$; we let $U_{(\alpha, \beta)}^{\beta} \subset I_{Y / T}(0, \alpha)$ be the image subset of this map, which is open. Because of this, we shall endow it with the induced scheme structure (usually non-reduced) from that of $I_{Y / T}(0, \alpha)$. For $\beta$, we have the similarly defined

$$
\varrho_{\beta}: Y_{(\beta, \alpha)}^{\llbracket \beta \rrbracket} \longrightarrow I_{Y / T}(0, \beta)
$$

induced by the families $\mathcal{W}_{i j}$. We then endow the open subset $U_{(\beta, \alpha)}^{\beta}=\operatorname{Im}\left(\varrho_{\beta}\right)$ with the induced scheme structure from that of $I_{Y / T}(0, \beta)$.

More to that, for any $\tilde{\eta} \in U_{(\beta, \alpha)}^{\beta}$ over $t \in T$ that is the image of an $\eta \in Y_{(\beta, \alpha)}^{\llbracket \beta \rrbracket}$, the associated (indexed) zero-subschemes $\eta_{11}, \cdots, \eta_{1 l_{1}}, \cdots, \eta_{k l_{k}} \subset Y_{t}$ for each $1 \leq i \leq k$ have that the collection $\eta_{i 1}, \cdots \eta_{i l_{i}}$ is mutually disjoint. Hence we can assign

$$
\xi_{i}=\eta_{i 1} \cup \cdots \cup \eta_{i l_{i}},
$$

thus obtaining a zero-subscheme in $I_{Y / T}\left(0,\left|\alpha_{i}\right|\right)$ and the tuple

$$
\left(\xi_{1}, \cdots, \xi_{k}\right) \in I_{Y / T}(0, \alpha)
$$

It is easy to see that this correspondence defines a one-one onto map from $U_{(\beta, \alpha)}^{\beta}$ to $U_{(\alpha, \beta)}^{\alpha}$.

The proof given in subSection 1.3 immediately shows that

Lemma 5.1 The induced map

$$
\Upsilon_{\alpha \beta}: U_{(\beta, \alpha)}^{\beta} \longrightarrow U_{(\alpha, \beta)}^{\alpha}
$$

is an étale morphism between two schemes; it commutes with the maps $\varrho_{\alpha}$, the map $\varrho_{\beta}$ and the partial equivalence $Y_{(\alpha, \beta)}^{\llbracket \alpha \rrbracket} \cong Y_{(\beta, \alpha)}^{\llbracket \beta \rrbracket}$. Further, the obstruction sheaves $\mathcal{O} b^{\llbracket \alpha \rrbracket}$ of $I_{Y / T}(0, \alpha)$ are isomorphic under pull back by $U_{(\beta, \alpha)}^{\beta}$ :

$$
\left.\Upsilon_{\alpha \beta}^{*} \mathcal{O} b_{Y}^{\llbracket \beta \rrbracket} \cong \mathcal{O} b_{Y}^{\llbracket \alpha \rrbracket}\right|_{Y_{(\alpha, \beta)}^{\alpha}} .
$$


The virtual normal cones are also identical under this isomorphism. We pick a locally free sheaf $\mathcal{E}_{\alpha}$ on $U_{(\alpha, \beta)}^{\alpha}$ that makes $\mathcal{O} b_{Y}^{[\alpha]}$ its quotient sheaf (over $U_{(\alpha, \beta)}^{\alpha}$ ). Then $\mathcal{E}_{\beta}=\Upsilon_{\alpha \beta}^{*} \mathcal{E}_{\alpha}$ is a locally free sheaf over $U_{(\beta, \alpha)}^{\beta}$ that makes $\mathcal{O} b_{Y}^{[\beta]}$ its quotient sheaf. Then the perfect-obstruction theory provides us the virtual normal cone $C_{\alpha} \subset E_{\alpha}$ and the normal cone $C_{\beta} \subset E_{\beta}$.

Lemma 5.2 Under the induced flat morphism $\phi_{\alpha \beta}: E_{\beta} \rightarrow E_{\alpha}$, the cycles

$$
\phi_{\alpha \beta}^{*} C_{\alpha}=C_{\beta} .
$$

Proof This follows from the uniqueness assertion on cones (22) and the following invariance result.

Lemma 5.3 Let $U \subset X$ be an open subset and let $\xi \subset U$ be a zero subscheme. Then the obstruction spaces to deforming $\xi$ in $U$ and in $X$ are canonically isomorphic. Further, under this isomorphism of obstruction spaces, the obstructions to deforming $\xi$ in $U$ and in $X$ are identical.

Proof First the to obstruction spaces are traceless extension groups

$$
\operatorname{Ext}_{X}^{2}\left(\mathcal{I}_{\xi}, \mathcal{I}_{\xi}\right)_{0} \quad \text { and } \quad \operatorname{Ext}_{U}^{2}\left(\mathcal{I}_{\xi}, \mathcal{I}_{\xi}\right)_{0}
$$

They are isomorphic because

$$
\operatorname{Ext}_{X}^{2}\left(\mathcal{I}_{\xi}, \mathcal{I}_{\xi}\right)_{0} \cong H^{0}\left(\mathcal{E} x t_{\mathcal{O}_{X}}^{3}\left(\mathcal{O}_{\xi}, \mathcal{I}_{\xi}\right)\right)=H^{0}\left(\mathcal{E} x t_{\mathcal{O}_{U}}^{3}\left(\mathcal{O}_{\xi}, \mathcal{I}_{\xi}\right)\right) \cong \operatorname{Ext}_{U}^{2}\left(\mathcal{I}_{\xi}, \mathcal{I}_{\xi}\right)_{0}
$$

As to the obstruction theory, say using locally free resolutions of $\mathcal{I}_{\xi}$ and using Cěch cohomology representative of the obstruction classes, one checks directly that the obstruction to deforming $\xi$ in $X$ gets mapped to the obstruction class to deforming $\xi$ in $U$ under the canonical homomorphism

$$
\operatorname{Ext}_{X}^{2}\left(\mathcal{I}_{\xi}, \mathcal{I}_{\xi}\right)_{0} \longrightarrow \operatorname{Ext}_{U}^{2}\left(\mathcal{I}_{\xi}, \mathcal{I}_{\xi}\right)_{0}
$$

But because this arrow is an isomorphism, the two obstruction classes must be identical.

\subsection{Some further notations}

From now on, for any $\alpha$ we fix a locally free sheaf $\mathcal{E}_{\alpha}$ over $I_{Y / T}(0, \alpha)$ that makes its quotient sheaf the obstruction sheaf $\mathcal{O} b^{[\alpha]}$ of $I_{Y / T}(0, \alpha)$; we let $C_{\alpha} \subset E_{\alpha}$ be the associated virtual normal cone. Because $Y^{[\alpha]}$ is $I_{Y / T}(0, \alpha)$ with reduced scheme structure, we can view $E_{\alpha}$ as a vector bundle over $Y^{[\alpha]}$ and view $C_{\alpha}$ as a cone cycle in $A_{*} E_{\alpha}$. 
For the Hilbert scheme of $\alpha$-points $Y \llbracket \alpha \rrbracket$, its obstruction sheaf $\mathcal{O} b_{Y}^{\llbracket \alpha \rrbracket}$ is canonically isomorphic to the pull back sheaf $\rho_{\alpha}^{*} \mathcal{O} b^{[\alpha]} \subset I_{Y / T}(0, \alpha)$ under the tautological morphism

$$
\rho_{\alpha}: Y^{\llbracket \alpha \rrbracket} \rightarrow Y^{[\alpha]} .
$$

Thus by taking $\widetilde{\mathcal{E}}_{\alpha}=\rho_{\alpha}^{*} \mathcal{E}_{\alpha}$, the obstruction sheaf $\mathcal{O} b_{Y}^{\llbracket \alpha \rrbracket}$ naturally becomes a quotient sheaf of $\widetilde{\mathcal{E}}_{\alpha}$.

We let $\widetilde{E}_{\alpha} \rightarrow E_{\alpha}$ be the tautological projection, viewed as a stack flat morphism

$$
\widetilde{E}_{\alpha}=E_{\alpha} \times_{Y^{(\alpha)}} Y^{\alpha} \longrightarrow E^{\Lambda} .
$$

The normal cone $\widetilde{C}_{\alpha} \subset \widetilde{E}_{\alpha}$ is then defined to be the stack flat pull back of $C_{\alpha}$ as specified in Definition 4.6.

For stratifications of $\widetilde{C}_{\alpha}$ and $Y \llbracket \alpha \rrbracket$, we shall take the standard pair of stratifications that respects the morphism $\widetilde{C}_{\alpha} \rightarrow Y^{\llbracket \alpha \rrbracket}$ and the loci of non-locally freeness of the sheaf $\mathcal{O} b_{Y}^{\llbracket \alpha \rrbracket}$.

Definition 5.4 We say that a smooth section $s_{\alpha} \subset \mathcal{A}\left(\mathcal{O} b_{\underset{\widetilde{\varepsilon}}{\llbracket \alpha} \rrbracket}^{\llbracket \rrbracket}\right)$ intersects transversally with its normal cone if one (thus all) of its lifts $\xi_{\alpha} \in \mathcal{A}\left(\widetilde{\mathcal{E}}_{\alpha}\right)$ intersects transversally with the cone $\widetilde{C}_{\alpha}$.

Following the discussion in subSection 4.3, we can find sections of $\mathcal{A}\left(\mathcal{O} b_{Y}^{\llbracket \alpha \rrbracket}\right)$ that intersect the normal cone transversally. For such $s_{\alpha}$, we shall denote by $D\left(s_{\alpha}\right) \subset Y \llbracket \alpha \rrbracket$ the pseudo-cycle that is the image in $Y \llbracket \alpha \rrbracket$ under $\widetilde{E}_{\alpha} \rightarrow Y \llbracket \alpha \rrbracket$ of intersecting a lift of $s_{\alpha}$ with $\widetilde{C}_{\alpha} \subset \widetilde{E}_{\alpha}$.

Now let $s_{\alpha} \in \mathcal{A}\left(\mathcal{O} b_{Y}^{\llbracket \alpha \rrbracket}\right)$ be a section that intersects transversally with the normal cone. Because $\left.\left.\mathcal{O} b_{Y}^{\llbracket \alpha \rrbracket}\right|_{Y_{(\alpha, \beta)}^{\llbracket \alpha \rrbracket}} \cong \mathcal{O} b_{Y}^{\llbracket \beta \rrbracket}\right|_{Y_{(\beta, \alpha)}^{\llbracket \beta \rrbracket}}$ canonically, we can view $s_{\alpha \mid \beta}=\left.s_{\alpha}\right|_{Y_{(\alpha, \beta)}^{\llbracket \alpha \rrbracket}}$ as a section of $\mathcal{O} b_{Y}^{\llbracket \beta \rrbracket}$ over $Y_{(\beta, \alpha)}^{\llbracket \beta \rrbracket}$. Because of Lemma 5.1 and 5.2, $s_{\alpha \mid \beta}$ is a smooth section of $\mathcal{O} b_{Y}^{\llbracket \beta \rrbracket}$ and intersects transversally with the normal cone of $Y \llbracket \beta \rrbracket$.

\subsection{Compatible cycle representatives}

To compare the cycles $\left[Y^{\llbracket \alpha \rrbracket}\right]^{\text {vir }}$, in this section we shall carefully pick smooth sections $s_{\alpha}$ so that for any pair $\alpha$ and $\beta$ the cycle representatives $D\left(s_{\alpha}\right)$ are $D\left(s_{\beta}\right)$ are identical over most part of the intersection $Y_{(\alpha, \beta)}^{\llbracket \alpha \rrbracket} \cong Y_{(\beta, \alpha)}^{\llbracket \beta \rrbracket}$.

To this end, we form the strict $\alpha$-diagonal

$$
\Delta_{\alpha}=\left\{x \in Y^{\Lambda} \mid a \sim_{\alpha} b \Rightarrow x_{a}=x_{b}\right\}
$$


they are closed; for different $\alpha$ and $\beta, \Delta_{\alpha} \cap \Delta_{\beta}=\Delta_{\alpha \vee \beta}{ }^{7}$. Next we fix a sufficiently small $c>0$ and pick a function $\varepsilon: \mathcal{P}_{\Lambda} \rightarrow(0, c)$ whose values on any ordered pair $\alpha>\beta$ obey $\varepsilon(\alpha)>R \varepsilon(\beta)$ for a sufficiently large $R$. After fixing a Riemannian metric on $Y$, we then form the $\varepsilon$-neighborhoods of $\Delta_{\alpha} \subset Y^{\Lambda}$ :

$$
\Delta_{\alpha, \varepsilon}=\left\{x \in Y^{\Lambda} \mid \operatorname{dist}\left(x, \Delta_{\alpha}\right)<\varepsilon(\alpha)\right\} .
$$

For any $\beta \leq \alpha$, we form

$$
\Delta_{\beta, \varepsilon}^{\alpha}=\bigcup_{\alpha \geq \gamma \geq \beta} \Delta_{\gamma, \varepsilon} \quad \text { and } \quad Q_{\beta, \varepsilon}^{\alpha}=\Delta_{\beta, \varepsilon}-\bigcup_{\alpha \geq \gamma>\beta} \Delta_{\gamma, \varepsilon}^{\alpha} .
$$

Note that $Q_{\beta, \varepsilon}^{\alpha}$ are closed subsets of $\Delta_{\beta, \varepsilon}$.

We have the following intersection property of these sets.

Lemma 5.5 For any pair $\beta_{1}, \beta_{2} \leq \alpha$ satisfying $\Delta_{\beta_{1}, \varepsilon} \cap Q_{\beta_{2}, \varepsilon}^{\alpha} \neq \varnothing$, necessarily $\beta_{2} \geq \beta_{1}$.

Proof Because $c$ is sufficiently small, whenever $\Delta_{\mu, \varepsilon} \cap \Delta_{v, \varepsilon} \neq \varnothing$, necessarily $\Delta_{\mu} \cap$ $\Delta_{v} \neq \varnothing$. Then because $\Delta_{\mu} \cap \Delta_{v}=\Delta_{\mu \vee v}$, because $\Delta_{\mu}$ intersects $\Delta_{v}$ perpendicularly, and because $\varepsilon(\mu \vee v)>\frac{R}{2} \varepsilon(\mu)+\frac{R}{2} \varepsilon(\nu)$,

$$
\Delta_{\mu, \varepsilon} \cap \Delta_{v, \varepsilon} \subset \Delta_{\mu \vee v, \varepsilon} .
$$

Now suppose $\beta_{2} \nsucceq \beta_{1}$, then $\beta_{1} \vee \beta_{2}>\beta_{2}$; therefore we have $\Delta_{\beta_{1}, \varepsilon} \cap \Delta_{\beta_{2}, \varepsilon} \subset \Delta_{\beta_{1} \vee \beta_{2}, \varepsilon}$ and $Q_{\beta_{2}, \varepsilon}^{\alpha} \subset \Delta_{\beta_{2}, \varepsilon}^{\alpha}-\Delta_{\beta_{1} \vee \beta_{2}, \varepsilon}^{\alpha}$. Combined, we have

$$
\varnothing \neq \Delta_{\beta_{1}, \varepsilon} \cap Q_{\beta_{2}, \varepsilon}^{\alpha} \subset \Delta_{\beta_{1} \vee \beta_{2}, \varepsilon} \cap\left(\Delta_{\beta_{2}, \varepsilon}-\Delta_{\beta_{1} \vee \beta_{2}, \varepsilon}\right)=\varnothing .
$$

This proves $\beta_{2} \geq \beta_{1}$.

An immediate corollary of this is that $\Delta_{\beta, \varepsilon}^{\alpha}=\bigsqcup_{\alpha \geq \gamma \geq \beta} Q_{\gamma, \varepsilon}^{\alpha}$ forms a partition (a disjoint union) of $\Delta_{\beta, \varepsilon}^{\alpha}$. By choosing $\beta=0_{\Lambda}$, it also shows that $\left\{Q_{\beta, \varepsilon}^{\alpha} \mid \beta \leq \alpha\right\}$ forms a partition of $Y^{\Lambda}$.

Moving to $Y^{\llbracket \alpha \rrbracket}$, we form

$$
\mathcal{N}_{\beta, \varepsilon}^{\alpha}=\rho_{\alpha}^{-1}\left(N_{\beta, \varepsilon}^{\alpha}\right) \quad \text { and } \quad \mathcal{Q}_{\beta, \varepsilon}^{\alpha} \triangleq \rho_{\alpha}^{-1}\left(Q_{\beta, \varepsilon}^{\alpha}\right) \subset Y^{\llbracket \alpha \rrbracket},
$$

in which we continue to denote by $\rho_{\alpha}: Y^{\llbracket \alpha \rrbracket} \longrightarrow Y^{\Lambda}$ the projection. The collection $\left\{\mathcal{Q}_{\beta, \varepsilon}^{\alpha} \mid \beta \leq \alpha\right\}$ forms a partition of $Y^{\llbracket \alpha \rrbracket}$.

${ }^{7} \alpha \vee \beta$ is the smallest element among all that are larger than or equal to both $\alpha$ and $\beta$. 
Lemma 5.6 For sufficiently small $c$, we can find a collection of sections $s_{\alpha} \in$ $\mathcal{A}_{\mathcal{S}}\left(\mathcal{O} b^{\llbracket \alpha \rrbracket}\right)$ that satisfy the properties

(i) each $s_{\alpha}$ intersects transversally with the normal cone of $\mathcal{O} b_{Y}^{\llbracket \alpha \rrbracket}$;

(ii) for any $\beta<\alpha$, the sections $s_{\alpha}$ and $s_{\beta}$ coincide over $\mathcal{Q}_{\beta, \varepsilon}^{\alpha}$.

The requirement (ii) is understood as follows. To each $\beta<\alpha$, because $\mathcal{Q}_{\beta, \varepsilon}^{\alpha}$ is disjoint from $\rho_{\alpha}^{-1}\left(\Delta_{\gamma}\right)$ for all $\alpha \geq \gamma>\beta$, it lies inside $Y_{(\alpha, \beta)}^{\llbracket \alpha \rrbracket}$. Thus restricting to $\mathcal{Q}_{\beta, \varepsilon}^{\alpha}$ both $s_{\alpha}$ and $s_{\beta}$ are sections of the same sheaf, and hence can be said to equal.

Proof We prove the lemma by induction. Because the space $Y \llbracket \alpha \rrbracket$ is a disjoint union of $\mathcal{Q}_{\beta, \varepsilon}^{\alpha}$, we will construct $s_{\alpha}$ by specifying its values along each of the above subsets according to (ii) and then showing that the resulting section can be extended to satisfy (i).

We now construct the section $s_{\alpha}$ by induction. Suppose we have already constructed $s_{\beta}$ for all $\beta<\alpha$ that satisfy the properties (i)-(ii). Along the partition $Y \llbracket \alpha \rrbracket=\bigsqcup_{\beta \leq \alpha} \mathcal{Q}_{\beta, \varepsilon}^{\alpha}$, we shall follow the rule (ii) to define

$$
s_{\alpha \mid \beta}=\left.s_{\beta}\right|_{\mathcal{Q}_{(\beta, \alpha)}^{\alpha}} \in \Gamma\left(\mathcal{Q}_{\beta, \varepsilon}^{\alpha}, \mathcal{A}_{\mathcal{S}}\left(\mathcal{O} b_{Y}^{\llbracket \alpha \rrbracket}\right)\right.
$$

be the restriction to $\mathcal{Q}_{\beta, \varepsilon}^{\alpha}$ of $s_{\beta}$. Inductively, this will define

$$
s_{\alpha} \in \Gamma\left(Y^{\llbracket \alpha \rrbracket}-\mathcal{N}_{\alpha, \varepsilon}^{\alpha}, \mathcal{A}_{\mathcal{S}}\left(\mathcal{O} b_{Y}^{\llbracket \alpha \rrbracket}\right)\right)
$$

after checking that the collection $s_{\alpha \mid \beta}$ forms a smooth section over $Y \llbracket \alpha \rrbracket-\mathcal{N}_{\alpha, \varepsilon}^{\alpha}$.

We now prove that it is so. First, because $Y^{\llbracket \alpha \rrbracket}-\mathcal{N}_{\alpha, \varepsilon}^{\alpha}$ is a disjoint union of $\left\{\mathcal{Q}_{\beta, \varepsilon}^{\alpha}\right.$ | $\beta<\alpha\}$, each $z \in Y \llbracket \alpha \rrbracket-\mathcal{N}_{\alpha, \varepsilon}^{\alpha}$ must lie in a $\mathcal{Q}_{\beta, \varepsilon}^{\alpha}$ for a unique $\beta<\alpha$. In case $z$ is an interior point of $\mathcal{Q}_{\beta, \varepsilon}^{\alpha}$, then $s_{\alpha}$ coincide with $s_{\beta}$ near $z$; by induction hypothesis, $s_{\alpha}$ satisfies the requirement (i) and (ii) near $z$. In case $z$ is not an interior point of $\mathcal{Q}_{\beta, \varepsilon}^{\alpha}$, since $\mathcal{N}_{\gamma, \varepsilon}^{\alpha}$ is open, by Lemma 5.5 this is possible only when $z$ lies in the closure $\operatorname{cl}\left(\mathcal{Q}_{\beta^{\prime}, \varepsilon}^{\alpha}\right)$ for some $\beta^{\prime}>\beta$. There are two possibilities: one is when $\beta^{\prime}=\alpha$, in which case nothing to prove. The other is when $\beta^{\prime}<\alpha$. Since $s_{\alpha}$ is defined via $s_{\beta}$ on $\mathcal{Q}_{\beta, \varepsilon}^{\alpha}$ and via $s_{\beta^{\prime}}$ on $\mathcal{Q}_{\beta^{\prime}, \varepsilon}^{\alpha}$, to check the continuity of $s_{\alpha}$, we need to compare the germ of $s_{\beta}$ and of $s_{\beta^{\prime}}$ near $z$. In this case, $\mathcal{Q}_{\beta^{\prime}, \varepsilon}^{\alpha} \subset Y_{(\alpha, \beta)}^{\llbracket \alpha \rrbracket}$, thus can be considered lies in $Y^{\llbracket \beta^{\prime} \rrbracket}$; as to $\mathcal{Q}_{\beta, \varepsilon}^{\alpha}, \mathcal{Q}_{\beta, \varepsilon}^{\alpha} \cap Y^{\llbracket \beta^{\prime} \rrbracket}=\mathcal{Q}_{\beta, \varepsilon}^{\beta^{\prime}}$. Hence by induction hypothesis, $s_{\beta^{\prime}}$ is an extension of $s_{\beta}$, Thus $s_{\alpha}$ is well-defined near $z$, and thus is smooth near $z$.

Once we know that $s_{\alpha}$ is smooth over $Y^{\llbracket \alpha \rrbracket}-\mathcal{N}_{\alpha, \varepsilon}^{\alpha}$, which is closed in $Y^{\llbracket \alpha \rrbracket}$, we can extend it to a good smooth section of $\mathcal{O} b_{Y}^{\llbracket \alpha \rrbracket}$ over $Y^{\llbracket \alpha \rrbracket}$, which completes the proof of the Lemma. 
From now on, we fix such a collection $\left\{s_{\alpha}\right\}_{\alpha \in \mathcal{P}_{\Lambda}}$ and form their associated pseudo-cycle representatives $D\left(s_{\alpha}\right)$.

\subsection{Approximation of the virtual cycles}

In this subsection, we shall investigate how pseudo-cycles $D\left(s_{\alpha}\right)$ are related by looking at their images in $Y^{\Lambda}$.

We let $d$ be the real dimension of $T$; let $D\left(s_{\alpha}\right)$ be the $d$-dimensional pseudo-cycles constructed relative to a set $\widetilde{\Theta}_{\alpha} \subset Y \llbracket \alpha \rrbracket$ of dimension $\leq d-2$. We take $\Theta \subset Y^{\Lambda}$ be the union of the images under the projection $\rho_{\alpha}: Y^{\llbracket \alpha \rrbracket} \rightarrow Y^{\Lambda}$ of all $\widetilde{\Theta}_{\alpha}: \Theta=\cup_{\alpha} \rho_{\alpha}\left(\widetilde{\Theta}_{\alpha}\right)$. Then to a sufficiently small $\epsilon>0$ independent of $c$, we pick $\tilde{f}_{\alpha}: \Sigma_{\alpha} \rightarrow Y \llbracket \alpha \rrbracket$ a representative of $D\left(s_{\alpha}\right)$ as $d$-dimensional pseudo-cycle relative to $\left(\rho_{\alpha}^{-1}(\Theta), \epsilon\right)$. The composition $f_{\alpha}=\rho_{\alpha} \circ \tilde{f}_{\alpha}$ defines a $(\Theta, \epsilon)$-relative pseudo-cycle, which we denote by $\left[f_{\alpha}\right]_{p c}$.

We now relate different pseudo-cycles $\left[f_{\alpha}\right]_{p c}$ by forming inductively

$$
\delta_{\alpha}=\left[f_{\alpha}\right]_{p c}-\sum_{\beta<\alpha} \delta_{\beta} \in C y c_{d}\left(Y^{\Lambda}\right)_{\Theta} .
$$

We have the following vanishing result:

Lemma 5.7 The pseudo-chain $\delta_{\alpha} \cap\left(Y^{\Lambda}-\Delta_{\alpha, 2 c}\right)$ is equivalent to 0 .

Proof What we need to show is that to any $z \in Y^{\Lambda}-\left(\Delta_{\alpha, 2 c} \cup \Theta_{\epsilon}\right)$ we can find a sufficiently small ball $B_{r}(z)$ centered at $z$ so that $\delta_{\alpha} \cap B_{r}(z)$ as a pseudo-cycle is equivalent to zero.

We now prove this by induction. For $\alpha=0_{\Lambda}$, there is nothing to prove. Now let $\alpha \in \mathcal{P}_{\Lambda}$ be any element so that this holds true for all $\beta<\alpha$. Let $x \in Y^{\Lambda}$ be any point away from $\Delta_{\alpha, 2 c} \cup \Theta_{\epsilon}$ and let $\beta<\alpha$ be so that

$$
x \in Q_{\beta, \varepsilon}^{\alpha} .
$$

We first claim that for sufficiently small $r, \delta_{\gamma} \cap B_{r}(x) \sim 0$ for all $\beta \nsucceq \gamma<\alpha$. Suppose not, then by induction hypothesis, $x \in \Delta_{\gamma, 2 c}$. Since $c$ is sufficiently small and $\varepsilon\left(\beta_{1}\right)>R \varepsilon\left(\beta_{2}\right)$ for any $\beta_{1}>\beta_{2}$, we have $\Delta_{\beta, \varepsilon} \cap \Delta_{\gamma, \varepsilon} \subset \Delta_{\beta \vee \gamma, \varepsilon}$, and hence $\rho(z) \in \Delta_{\beta \vee \gamma, \varepsilon}$. Because we have assumed that $\beta \nsucceq \gamma$, we must have $\beta \vee \gamma>\beta$, which implies that $\Delta_{\beta \vee \gamma, \varepsilon} \cap Q_{\beta, \varepsilon}^{\alpha}=\varnothing$, contradicting to $x \in Q_{\beta, \varepsilon}^{\alpha}$ and in $\Delta_{\beta \wedge \gamma, \varepsilon}$.

Because of this, when we intersects $\delta_{\alpha}$ with $B_{r}(x)$, for sufficiently small $r$ (25) tells us that

$$
\delta_{\alpha} \cap B_{r}(x)=\left[f_{\alpha}\right]_{p c} \cap B_{r}(x)-\sum_{\gamma \leq \beta} \delta_{\gamma} \cap B_{r}(x) .
$$


On the other hand by definition

$$
\delta_{\beta} \cap B_{r}(x)=\left[f_{\beta}\right]_{p c} \cap B_{r}(x)-\sum_{\gamma<\beta} \delta_{\gamma} \cap B_{r}(x) .
$$

Hence if we can show that $\left[f_{\alpha}\right]_{p c} \cap B_{r}(x)=\left[f_{\beta}\right]_{p c} \cap B_{r}(x)$, the above two identities will force $\delta_{\alpha} \cap B_{r}(x)=0$, exactly what we intend to prove.

For this we argue as follows: let $z \in D\left(s_{\alpha}\right) \cap \rho_{\alpha}^{-1}\left(B_{r}(x)\right)$. Because of (26), $x \in \Delta_{\beta, \varepsilon}$ and $x \notin \cup_{\beta<\gamma \leq \alpha} \Delta_{\gamma}$; thus $z$ must lie in $Y_{(\alpha, \beta)}^{\llbracket \alpha \rrbracket}$ and thus also in $Y_{(\beta, \alpha)}^{\llbracket \beta \rrbracket}$. Hence (ii) of lemma 5.6 implies that

$$
D\left(s_{\alpha}\right) \cap \rho_{\alpha}^{-1}\left(B_{r}(x)\right)=D\left(s_{\beta}\right) \cap \rho_{\beta}^{-1}\left(B_{r}(x)\right) .
$$

For the same reason, the above identity holds in case $x \in D\left(s_{\beta}\right)$. This proves the lemma.

\subsection{Truncated discrepancy cycles}

Because $\delta_{\alpha}$ is equivalent to zero away from $\Delta_{\alpha, 2 c}$, we can truncate it by intersecting it with the closed subset $\bar{\Delta}_{\alpha, e}$ with a general $2 c<e<3 c: \delta_{\alpha}^{e}=\delta_{\alpha} \cap \bar{\Delta}_{\alpha, e}$. It can also be defined inductively by

$$
\delta_{\alpha}^{e} \sim\left[f_{\alpha}\right]_{p c} \cap \bar{\Delta}_{\alpha, e}-\sum_{\beta<\alpha} \delta_{\beta}^{e},
$$

where the intersection $\left[f_{\alpha}\right]_{p c} \cap \bar{\Delta}_{\alpha, e}$ can be replaced by any pseudo-chain $\left.f_{\alpha}\right|_{\Sigma_{\alpha}^{e}}$ with $\Sigma_{\alpha}^{e}=f_{\alpha}^{-1}\left(\bar{\Delta}_{\alpha, e}\right)-O$ for an open $O$ such that $\Sigma_{\alpha}^{e}$ has smooth boundary and $f_{\alpha}(O) \subset \Theta_{\varepsilon}$. By Sards theorem, for general $e$ the set $f_{\alpha}^{-1}\left(\bar{\Delta}_{\alpha, e}\right)$ has smooth boundary away from $f_{\alpha}^{-1}\left(\Theta_{\varepsilon}\right)$. Thus we can choose $e$ that works for all $\alpha$.

Lemma 5.8 With such choice of $e$ and pseudo-chain representative $\left[f_{\alpha}\right]_{p c} \cap \bar{\Delta}_{\alpha, e}$, the inductively defined pseudo chain $\delta_{\alpha}^{e}$ is a pseudo-cycle relative to $(\Theta, \epsilon)$.

Proof This follows directly from Lemma 5.7 and the definition of pseudo-cycle.

In case $\alpha$ has more than one equivalence class, the cycle $\delta_{\alpha}$ is equivalent to the product of $\delta_{\alpha_{i}}$. More precise, by viewing each $\alpha_{i}$ as a set, we can form the spaces $Y^{\alpha_{i}}$ and the pseudo-cycles $\delta_{\beta_{i}}$ for $\beta_{i} \in \mathcal{P}_{\alpha_{i}}$. We continue to denote by $\alpha_{i}$ the top partition in $\mathcal{P}_{\alpha_{i}}$.

Lemma 5.9 We can choose representatives $\delta_{\alpha_{i}}$ and $\delta_{\alpha}$ so that under the identity $Y^{\Lambda}=\prod_{T}^{k} Y^{\alpha_{i}}$ as pseudo-cycles $\delta_{\alpha} \cong \prod_{i=1}^{k} \delta_{\alpha_{i}}$. 
Proof For each $\alpha_{i}$, we form the cycle representatives $D(\gamma) \subset Y \llbracket \gamma \rrbracket$ and the discrepancy cycles $\delta_{\gamma}$ by picking a collection of sections $\left\{s_{\gamma} \mid \gamma \in \mathcal{P}_{\alpha_{i}}\right\}$ provided by the previous lemma. For each $\beta \leq \alpha$ in $\mathcal{P}_{\Lambda}$, we write $\beta$ as $\left(\beta_{11}, \cdots \beta_{k l_{k}}\right)$ and form $\beta_{i}=\left(\beta_{i 1}, \cdots, \beta_{i l_{i}}\right)$, each is a partition in $\mathcal{P}_{\alpha_{i}}$. Then

$$
Y^{\llbracket \beta \rrbracket}=Y^{\llbracket \beta_{1} \rrbracket} \times \cdots \times Y^{\llbracket \beta_{k} \rrbracket} \quad \text { and } \quad \mathcal{O} b_{Y}^{\llbracket \beta \rrbracket}=\pi_{1}^{*} \mathcal{O} b_{Y}^{\llbracket \beta_{1} \rrbracket} \oplus \cdots \oplus \pi_{k}^{*} \mathcal{O} b_{Y}^{\llbracket \beta_{k} \rrbracket}
$$

with $\pi_{i}$ the $i$-th projection of $Y \llbracket \alpha \rrbracket$ to $Y \llbracket \alpha_{i} \rrbracket$. Further, the sections $s_{\beta_{1}}, \cdots, s_{\beta_{k}}$ provides us a section

$$
s_{\beta}=\pi_{1}^{*} s_{\beta_{1}} \oplus \cdots \oplus \pi_{k}^{*} s_{\beta_{k}}
$$

with associated pseudo-cycle

$$
D\left(s_{\beta}\right)=D\left(s_{\beta_{1}}\right) \times \cdots \times D\left(s_{\beta_{k}}\right) .
$$

We claim that using such decomposition, the discrepancy pseudo-cycles

$$
\delta_{\beta} \cong \delta_{\beta_{1}} \times \cdots \times \delta_{\beta_{k}} .
$$

Indeed, when $\beta=0_{\Lambda}$, then the identity reduces to

$$
D\left(s_{\beta}\right)=\prod_{a \in \Lambda} D\left(s_{1_{\{a\}}}\right),
$$

which is (27). Now suppose the identity holds for all $\gamma<\beta$. Then

$$
\begin{aligned}
\prod_{i=1}^{k} D\left(s_{\beta_{i}}\right) & =\prod_{i=1}^{k}\left(\sum_{\beta_{i j} \leq \beta_{i}} \delta_{\beta_{i j}}\right)=\sum_{\left(\gamma_{1}, \cdots, \gamma_{k}\right) \in \mathcal{P}_{\beta_{1}} \times \cdots \times \mathcal{P}_{\beta_{k}}} \delta_{\gamma_{1}} \times \cdots \times \delta_{\gamma_{k}} \\
& =\sum_{\gamma<\beta} \delta_{\gamma}+\delta_{1_{\beta_{1}}} \times \cdots \times \delta_{1_{\beta_{k}}} .
\end{aligned}
$$

The desired identity (28) then follows from $D\left(s_{\beta}\right)=\sum_{\gamma<\beta} \delta_{\gamma}+\delta_{\beta}$ and the identity (27). This proves the Lemma.

By choosing a general $e$ as before, the truncated discrepancy cycle also satisfies the product formula (28) with $\delta$. replaced by its truncated version $\delta$. .

\section{Proof of the Main theorem}

The proof of the theorem now is fairly straightforward. We first apply the previous construction to $Y=X$ and to the set $\Lambda=[n]$ of integers from 1 to $n$ to relate the 
degree of the virtual cycle

$$
\operatorname{deg}\left[I_{X}(0, n)\right]^{\mathrm{vir}}=\frac{1}{n !} \operatorname{deg}\left[X^{\llbracket n \rrbracket}\right]^{\mathrm{vir}} .
$$

(Here we follow the convention $X^{\llbracket n \rrbracket}=X^{\llbracket \Lambda \rrbracket}$.)

The right hand side can be expressed as the degree of an explicit zero-cycle. For this we pick a smooth section $s_{[n]}$ of the obstruction sheaf $\mathcal{O} b_{X}^{\llbracket n \rrbracket}$ of $X^{\llbracket n \rrbracket}$ that intersects transversally with the normal cone. Because the virtual dimension of $I_{X}(0, n)$ is zero, the resulting pseudo-cycle $D\left(s_{[n]}\right)$ in $X^{\llbracket n \rrbracket}$ is a zero-cycle, satisfying

$$
\operatorname{deg}\left[X^{\llbracket n \rrbracket}\right]^{\mathrm{vir}}=\operatorname{deg}\left[D\left(s_{[n]}\right)\right] .
$$

To proceed, we shall relate it to the degrees of the discrepancy cycles $\delta_{\alpha}$. For a sufficiently small $\epsilon$ and $c$ and for all $\alpha \in \mathcal{P}_{\Lambda}$ we choose sections $s_{\alpha}$ of $\mathcal{O} b_{X}^{\llbracket \alpha \rrbracket}$ according to Lemma 5.6. We let $D\left(s_{\alpha}\right)$ in $X^{\llbracket \alpha \rrbracket}$ be the associated zero-cycle derived by intersecting the normal cone by $s_{\alpha}$. However, because each $\delta_{\alpha}$ is a zero-cycle, Lemma 5.7 shows that it is entirely contained in the $\epsilon$-tubular neighborhood of $X_{\Delta}^{n} \subset X^{n}$. Further, their degrees

$$
\operatorname{deg}\left[D\left(s_{[n]}\right)\right]=\sum_{\alpha \in \mathcal{P}_{\Lambda}} \operatorname{deg} \delta_{\alpha} .
$$

Thus to prove that $\operatorname{deg}\left[I_{X}(0, n)\right]^{\text {vir }}$ is expressible in terms of a universal polynomial in Chern numbers of $X$ we only need to show that the same hold for all $\operatorname{deg} \delta_{\alpha}$, which then follows from that of $\operatorname{deg} \delta_{[n]}$ by the product formula in Lemma 5.9. This way, we are reduced to show that $\operatorname{deg} \delta_{[n]}$ is expressible by a universal expression in Chern numbers of $X$.

Our next step is to use the construction of the diffeomorphism of a neighborhood of $0_{T X} \subset T X$ with a neighborhood of the diagonal $\Delta(X) \subset X \times X$ to transfer the cycle $\delta_{[n]}$ to the tangent bundle $T X$.

We let $\mathcal{U} \subset X \times X$ be the tubular neighborhood of $\Delta(X) \subset X \times X$, let $\mathcal{V} \subset T X$ be the tubular neighborhood of $0_{T X} \subset T X$ and let $\psi: \mathcal{V} \rightarrow \mathcal{U}$ be the smooth isomorphism provided by Lemma 2.4. The map $\psi$ induces a smooth isomorphism

$$
\Psi_{\alpha}: \mathcal{V}_{0}^{\llbracket \alpha \rrbracket} \longrightarrow U^{\llbracket \alpha \rrbracket} \subset X^{\llbracket \alpha \rrbracket}
$$

onto an open neighborhood of $X_{\Delta}^{\llbracket \alpha \rrbracket} \subset X^{\llbracket \alpha \rrbracket}$ (see Lemma 2.6). Because $U^{\llbracket \alpha \rrbracket}$ is an open neighborhood of $X_{\Delta}^{\llbracket \alpha \rrbracket}$, by choosing $c$ sufficiently small, Lemma 5.7 tells us that the cycle $\delta_{[n]}$ lies entirely in $U^{\llbracket n \rrbracket}$. Thus the degrees of

$$
\delta_{[n], \mathcal{V}}=\Psi_{[n]}^{*}\left(\delta_{[n]}\right)
$$


is identical to that of $\delta_{[n]}$.

The cycle $\delta_{[n], \mathcal{V}}$ can also be constructed using the universal family over the Grassmannian of quotients $\mathbb{C}^{N} \rightarrow \mathbb{C}^{3}$. Let $Q \rightarrow G r$ be the total space of the universal quotient bundle and let $Q^{\llbracket \alpha \rrbracket}$ (resp. $Q_{0}^{\llbracket \alpha \rrbracket}$ ) be the relative Hilbert scheme of $\alpha$-points (resp. centered $\alpha$-points) of $Q / G r$. To each $Q_{0}^{\llbracket \alpha \rrbracket}$ we form its obstruction sheaf $\mathcal{O} b_{Q}^{\llbracket \alpha \rrbracket}$; we pick a locally free sheaf $\mathcal{E}_{\alpha}$ making $\mathcal{O} b_{Q}^{\llbracket \alpha \rrbracket}$ its quotient sheaf; we let $E_{\alpha}$ be the associated vector bundle and let $D_{\alpha} \subset E_{\alpha}$ be the associated normal cone.

We then pick a smooth section $t_{\alpha}$ of $E_{\alpha}$ for all $\alpha$ so that the collection $\left\{t_{\alpha}\right\}_{\alpha \in \mathcal{P}_{\Lambda}}$ satisfies the conclusion of Lemma 2.4. The sections $t_{\alpha}$ provide us the pseudo-cycle $D\left(t_{\alpha}\right)$ in $Q_{0}^{\llbracket \alpha \rrbracket}$ relative to a subset $\widetilde{\Theta}_{\alpha}$ of dimension at most $2 \operatorname{dim} G r-8$. According to (25), the images under the projections $\eta_{\alpha}: Q_{0}^{\llbracket \alpha \rrbracket} \rightarrow Q_{0}^{n}$ of the pseudo-cycles $D\left(t_{\alpha}\right)$ form the discrepancy pseudo-cycle $\delta_{[n], Q} \subset Q_{0}^{n}$ relative to $(\Theta, \epsilon)$, the set $\Theta$ which is the union of all $\eta_{\alpha}\left(\widetilde{\Theta}_{\alpha}\right)$ and for $\epsilon$ which is sufficiently small.

The cycle $\delta_{[n], Q}$ defines a codimension six homology class in $G r$. Indeed, by Lemma 5.7, the pseudo-cycle is zero outside the $2 c$-tubular neighborhood in $Q_{0}^{n}$ of the top diagonal $Q_{\Delta}^{n} \cap Q_{0}^{n}$. Because the top diagonal $Q_{\Delta}^{n}$ consists of points $(\xi, \cdots, \xi)$, they are centered only if $\xi=0$. Thus the top diagonal is the zero section of $Q_{0}^{n} / G r$; thus is compact. Therefore, $\delta_{[n], Q}$ is a compact pseudo-cycle, thus defines a homology class

$$
\left[\delta_{[n], Q} \in H_{\operatorname{dim}_{\mathbb{R}}} G r-6\left(Q_{0}^{n}, \mathbb{Z}\right) \cong H_{\operatorname{dim}_{\mathbb{R}} G r-6}(G r, \mathbb{Z}) .\right.
$$

We shall relate the cycle $\delta_{[n], Q}$ with $\delta_{[n]}$ in $X^{n}$ by picking a smooth map $g: X \rightarrow G r$ so that as smooth vector bundle $g^{*} Q=T X$. Without loss of generality, we can choose $N$ to be sufficiently large and choose $g$ to be an embedding. Obviously, $g$ induces a smooth isomorphism (compare to (12))

$$
g_{n}: Q_{0}^{n} \times_{G r} X \cong(T X)_{0}^{n} .
$$

We have the following compatibility result.

Lemma 6.1 We can choose $g$ and sections $s_{\alpha}$ so that $Q_{0}^{n} \times_{G r} X$ intersects transversally with $\delta_{[n], Q}$ and the intersection

$$
g_{n}^{*}\left(\delta_{[n], Q}\right) \triangleq \delta_{[n], Q} \cap\left(Q_{0}^{n} \times_{G r} X\right),
$$

considered as a cycle in $(T X)_{0}^{n}$ via the isomorphism (30), is identical to the cycle $\delta_{[n], \mathcal{V}}$ via the inclusion $\mathcal{V}_{0}^{n} \subset(T X)_{0}^{n}$. 
Once the lemma is proved, then we can use the constructed sections $s_{\alpha}$ to form the cycle $\delta_{[n]}$ in $X^{n}$ to conclude

$$
\operatorname{deg} \delta_{[n]}=\operatorname{deg} \delta_{[n], \mathcal{V}}=\operatorname{deg} g_{n}^{*}\left(\delta_{[n], Q}\right)=\operatorname{deg}\left(\left[\delta_{[n], Q}\right]^{P . D .} \cap g_{*}([X])\right),
$$

where $\left[\delta_{[n], Q}\right]^{P . D .}$ is the Poincare dual of the homology class $\left[\delta_{[n], Q}\right] \in H_{6}(G r, \mathbb{Z})$. Because $H^{*}(G r, \mathbb{Z})$ is generated by $u_{i} \in H^{2 i}(G r, \mathbb{Z})$,

$$
\left[\delta_{[n], Q}\right]^{P . D .}=P_{n}\left(u_{1}, u_{2}, u_{3}\right) \in H^{6}(G r, \mathbb{Z})
$$

expressible in a polynomial in $u_{i}$. On the other hand, using the embedding $\operatorname{Gr}(N, 3) \subset$ $\operatorname{Gr}(N+1,3)$ and the proof that will follow, we see immediately that this polynomial $P_{n}$ is independent of the choice of $N$; thus is universal in $n$.

Finally, because $g_{n}^{*}\left(u_{i}\right)=c_{i}(X)$,

$$
\left[\delta_{[n], Q}\right]^{P . D .} \cap g_{*}([X])=p_{n}\left(c_{1}(X), c_{2}(X), c_{3}(X)\right) \cap[X]
$$

is a universal expression in the Chern numbers of $X$. This proves the main theorem.

We shall divide the proof of Lemma 6.1 into two steps: one is to compare the intersection $g_{n}^{*}\left(\delta_{[n], Q}\right)$ with a similarly constructed cycle $\delta_{[n], T X}$ on $(T X)_{0}^{n}$; the other is to compare the later with the pull back of $\delta_{[n]}$ using the map $\mathcal{V}_{0}^{n} \rightarrow X^{n}$. Since the proofs of both are similar, we shall provide the details of the first while indicating the necessary changes required for the second.

We begin with a quick account of the moduli of $\mathbf{A}^{3}$. We let $V=\mathbf{A}^{3}$ and let $V_{0}^{\llbracket \alpha \rrbracket} \rightarrow V_{0}^{n}$ be the tautological map from the Hilbert scheme of centered $\alpha$-points to $V_{0}^{n}$ that was constructed in section 1 with $Y$ replaced by $V$. Because $G L(3)$ acts on $V$, it acts on $V^{\llbracket \alpha \rrbracket}$ and $V^{n}$. Further these actions leave the projection $V \llbracket \alpha \rrbracket \rightarrow V^{n}$ and the averaging map $V^{n} \rightarrow V$ invariant, thus the space $V_{0}^{\llbracket \alpha \rrbracket}=V^{\llbracket \alpha \rrbracket} \times_{V} 0$ is $G L(3)-$ invariant. Not only that, its obstruction sheaf $\mathcal{O} b_{V}^{\llbracket \alpha \rrbracket}$ and its obstruction theory are all naturally $G L(3)$-linearized.

Because $G L(3)$ is reductive, we can find a $G L(3)$-linearized locally free sheaf $\mathcal{F}_{\alpha}$ on $V_{0}^{\llbracket \alpha \rrbracket}$ that makes $\mathcal{O} b_{V}^{\llbracket \alpha \rrbracket}$ its $G L(3)$-equivariant quotient sheaf

$$
\mathcal{F}_{\alpha} \longrightarrow \mathcal{O} b_{V}^{\llbracket \alpha \rrbracket} \text {. }
$$

We let $F_{\alpha}$ be its associated vector bundle and let $D_{\alpha} \subset F_{\alpha}$ be the associated normal cone. Then each $D_{\alpha, i}$ in the irreducible decomposition

$$
D_{\alpha}=\sum_{i} m_{\alpha, i} D_{\alpha, i}
$$


is a $G L(3)$-invariant cone-like subvariety of $F_{\alpha}$.

We next fix a standard stratification of $\cup_{i} D_{\alpha, i} \rightarrow V_{0}^{\llbracket \alpha \rrbracket}$ subordinating to the loci of the non-locally freeness of the sheaf $\mathcal{O} b_{V}^{\llbracket \alpha \rrbracket}$. To each $D_{\alpha, i}$, we let $S_{\alpha, i} \subset D_{\alpha, i}$ be its open stratum and let $T_{\alpha, i} \subset V_{0}^{\llbracket \alpha \rrbracket}$ be the image of $S_{\alpha, i}$, which is a stratum of $V_{0}^{\llbracket \alpha \rrbracket}$. Because $D_{\alpha, i} \rightarrow F_{\alpha} \rightarrow V_{0}^{\llbracket \alpha \rrbracket}$ are $G L(3)$-equivariant, every stratum, including $S_{\alpha, i}$, are $G L(3)$-invariant.

Our next step is to use (31) to build locally free sheaves on $Q_{0}^{\llbracket \alpha \rrbracket}$ and on $(T X)_{0}^{\llbracket \alpha \rrbracket}$ making their respective obstruction sheaves their quotient. For $Q / G r$, we first cover $G r$ by open $U_{a} \subset G r$ with vector bundle isomorphisms

$$
f_{a}: Q \times_{G r} U_{a} \stackrel{\cong}{\longrightarrow} V \times U_{a} .
$$

Then using the induced isomorphism

$$
Q_{0}^{\llbracket \alpha \rrbracket} \times_{G r} U_{a} \cong V_{0}^{\llbracket \alpha \rrbracket} \times U_{a}
$$

and $p_{V}$ the first projection of the product on the right hand side, we can form the induced

$$
\mathcal{E}_{\alpha, a}=\left.p_{V}^{*} \mathcal{F}_{\alpha} \longrightarrow p_{V} \mathcal{O} b_{V}^{\llbracket \alpha \rrbracket} \cong \mathcal{O} b_{Q}^{\llbracket \alpha \rrbracket}\right|_{Q_{0}^{\llbracket \alpha \rrbracket} \times_{G r} U_{a}}
$$

Over $U_{a b}=U_{a} \cap U_{b}$, the isomorphisms

$$
f_{b a}=f_{b} \circ f_{a}^{-1}: V \times U_{a b} \longrightarrow V \times U_{a b}
$$

induce transition isomorphisms

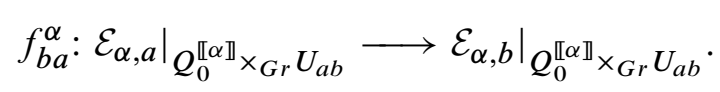

Since $f_{a b}$ satisfy the cocycle condition, $f_{a b}^{\alpha}$ also satisfy the cocycle condition. Hence $f_{a b}^{\alpha}$ glue to form a locally free sheaf $\mathcal{E}_{\alpha}$ on $Q_{0}^{\llbracket \alpha \rrbracket}$. Obviously, the quotient homomorphisms (33) glue to form a quotient homomorphism

$$
\mathcal{E}_{\alpha} \longrightarrow \mathcal{O} b_{Q}^{\llbracket \alpha \rrbracket}
$$

The normal cone of $\mathcal{O} b_{Q}^{\llbracket \alpha \rrbracket}$ takes a simple form in this setting. Let $E_{\alpha}$ be the associated vector bundle of $\mathcal{E}_{\alpha}$. The cycles

$$
D_{\alpha} \times U_{a}=\sum_{i} m_{\alpha, i} D_{\alpha, i} \times U_{a} \in C_{*}\left(F_{\alpha} \times U_{a}\right)=C_{*}\left(\left.E_{\alpha}\right|_{Q_{0}^{\llbracket \alpha \rrbracket_{\times_{G r}} U_{a}}}\right)
$$


glue together to form a cycle that is the normal cone $C_{\alpha} \subset E_{\alpha}$ :

$$
C_{\alpha}=\sum_{i} m_{\alpha, i} C_{\alpha, i}
$$

with $C_{\alpha, i}$ the glued subvariety from $D_{\alpha, i} \times U_{a}$.

Similarly, for the vector bundle $T X / X$ we can carry over the same procedure to form a locally free sheaf $\widetilde{\mathcal{E}}_{\alpha}$ over $(T X)_{0}^{\llbracket \alpha \rrbracket}$ making the obstruction sheaf $\mathcal{O} b_{T X}^{\llbracket \alpha \rrbracket}$ its quotient sheaf; the normal cone in the associate vector bundle $\widetilde{C}_{\alpha} \subset \widetilde{E}_{\alpha}$ is also the similarly induced cycle by $D_{\alpha} \subset F_{\alpha}$.

Now we look at the smooth map $g: X \rightarrow G r$, which we assume to be an embedding, and the smooth isomorphism

$$
g_{0}: T X \cong Q \times_{G r} X \subset Q .
$$

We let

$$
g_{\alpha}:(T X)_{0}^{\llbracket \alpha \rrbracket} \longrightarrow Q_{0}^{\llbracket \alpha \rrbracket}
$$

be the induced smooth map; we let $\mathcal{A}_{1}$ and $\mathcal{A}_{2}$ be the sheaves of smooth functions of $(T X)_{0}^{\llbracket \alpha \rrbracket}$ and $Q_{0}^{\llbracket \alpha \rrbracket}$ respectively. We claim that there are smooth isomorphisms as shown below that make the diagram commutative

$$
\begin{array}{ccc}
g_{\alpha}^{*}\left(\mathcal{E}_{\alpha} \otimes \mathcal{A}_{2}\right) & \longrightarrow g_{\alpha}^{*}\left(\mathcal{O} b_{Q}^{\llbracket \alpha \rrbracket} \otimes \mathcal{A}_{2}\right) \\
\cong \downarrow & \cong \downarrow \\
\tilde{\mathcal{E}}_{\alpha} \otimes \mathcal{A}_{1} & \longrightarrow \mathcal{O} b_{T X}^{\llbracket \alpha \rrbracket} \otimes \mathcal{A}_{1} .
\end{array}
$$

Indeed, for any open $U_{a} \subset X$ with trivialization $\left.T X\right|_{U_{a}} \cong V \times U_{a}$ and open $U_{b} \subset G r$ with trivialization $\left.Q\right|_{U_{b}} \cong V \times U_{b}$ and satisfying $g\left(U_{a}\right) \subset U_{b}$, the isomorphism $g_{0}$ in (34) defines a smooth map $h: U_{a} \rightarrow G L(V)$ that makes the diagram commutative

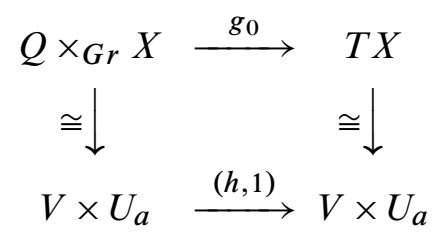

The family of automorphisms $h$ then induce smooth diffeomorphism

$$
\begin{array}{cc}
V_{0}^{\llbracket \alpha \rrbracket} \times U_{a} \stackrel{\left(h_{\alpha}, 1\right)}{\longrightarrow} \quad V_{0}^{\llbracket \alpha \rrbracket} \times U_{a} \\
\cong \downarrow \\
Q_{0}^{\llbracket \alpha \rrbracket} \times_{G r} U_{a} \stackrel{\cong \downarrow}{\cong}(T X)_{0}^{\llbracket \alpha \rrbracket} \times_{X} U_{a}
\end{array}
$$


and isomorphisms of sheaves

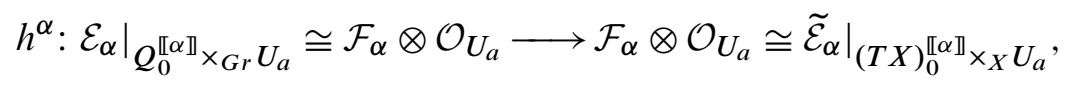

where the first and the third isomorphisms are induced by the construction of $\mathcal{E}_{\alpha}$ and $\widetilde{\mathcal{E}}_{\alpha}$ in (33) while the middle one is induced by $h$.

The homomorphisms $h^{\alpha}$ for a covering of $X$ patch together to form the left smooth isomorphism in (35); it makes that diagram commutative.

This way, by lifting $t_{\alpha}$ to a smooth section in $\mathcal{E}_{\alpha}$, then pulling the lifted section back to a smooth section of $\widetilde{\mathcal{E}}_{\alpha}$, and finally pushing forward the new section to a section in the obstruction sheaf $\mathcal{O} b_{T X}^{\llbracket \alpha \rrbracket}$, we obtain a smooth section. Obviously, the resulting section the original pull back $\widetilde{t}_{\alpha}$; therefore, $\widetilde{t}_{\alpha}$ is smooth. Likewise, because $g: X \rightarrow G r$ is a smooth embedding, because each stratum of $C_{\alpha, i}$ is submersive onto $G r$, and because as subsets

$$
\left.E_{\alpha}\right|_{Q_{0}^{\llbracket \alpha \mathbb{1}_{\mathrm{G} X} X}} \supset D_{\alpha, i} \cap\left(Q_{0}^{\llbracket \alpha \rrbracket} \times_{G r} X\right)=\widetilde{D}_{\alpha, i} \subset \widetilde{E}_{\alpha}
$$

under the isomorphism (35), we see immediately that a lift of $\tilde{t}_{\alpha}$ intersects transversally with the cone cycle $\widetilde{C}_{\alpha}$ if and only if $Q_{0}^{\llbracket \alpha \rrbracket} \times_{G r} X$ intersects transversally with the pseudo-cycle $D\left(t_{\alpha}\right)$. But this is possible if we choose $g$ general. Therefore, we can choose $g$ that makes all pull back sections $\tilde{t}_{\alpha}$ satisfy the first conclusion of Lemma 5.6. Since the collection $t_{\alpha}$ satisfies the second conclusion of Lemma 5.6, so does the collection $\tilde{t}_{\alpha}$.

Apply the same argument to the standard embedding

$$
G_{N}: \operatorname{Gr}(N, 3) \longrightarrow \operatorname{Gr}(N+1,3)
$$

we see that we can choose sections $t_{\alpha}$ 's so that

$$
\delta_{[n], G r(N, 3)}=g_{N}^{*}\left(\delta_{[n], G r(N+1,3)}\right)
$$

are stable under $G_{N}$. Therefore, their homology classes

$$
\left[\delta_{[n], G r}(N, 3)\right] \in H_{6}(\operatorname{Gr}(N, 3) ; \mathbb{Z})
$$

is stable under inclusions.

It remains to use the sections $\tilde{t}_{\alpha}$ to get smooth sections $s_{\alpha}$ of $\mathcal{O} b_{X}^{\llbracket \alpha \rrbracket}$. First, because $\mathcal{V}_{0}^{\llbracket \alpha \rrbracket}$ is an open subset of $(T X)_{0}^{\llbracket \alpha \rrbracket}$, the section $\tilde{t}_{\alpha}$ restricts to a section of the obstruction sheaf $\mathcal{O} b_{\mathcal{V}}^{\llbracket \alpha \rrbracket}$ of $\mathcal{V}_{0}^{\llbracket \alpha \rrbracket}$. Without much confusion, we shall denote the restriction section by $\tilde{t}_{\alpha}$ as well. 
Our next step is to take the induced sections $\tilde{s}_{\alpha}$ of $\left.\mathcal{O} b_{X}^{\llbracket \alpha \rrbracket}\right|_{U \llbracket \alpha \rrbracket}$ under the smooth isomorphisms

$$
\Psi_{\alpha}^{*} \mathcal{O} b_{X}^{\llbracket \alpha \rrbracket} \cong \mathcal{O} b_{\mathcal{V}}^{\llbracket \alpha \rrbracket}
$$

covering the smooth isomorphism $\Phi_{\alpha}: \mathcal{V}_{0}^{\llbracket \alpha \rrbracket} \rightarrow U^{\llbracket \alpha \rrbracket} \subset X^{\llbracket \alpha \rrbracket}$ in (29) and show that they are smooth and satisfy the conclusion of Lemma 5.6.

We begin with more notations for $X^{\llbracket \alpha \rrbracket}$. We pick a locally free sheaf $\overline{\mathcal{E}}_{\alpha}$ on $X^{\llbracket \alpha \rrbracket}$ making $\mathcal{O} b_{X}^{\llbracket \alpha \rrbracket}$ its quotient sheaf; we let $\bar{C}_{\alpha} \subset \bar{E}_{\alpha}$ be their associated normal cones with irreducible decomposition

$$
\bar{C}_{\alpha}=\sum \bar{m}_{\alpha, i} \bar{C}_{\alpha, i}
$$

Like before, we denote by $\bar{S}_{\alpha, i} \subset \bar{C}_{\alpha, i}$ its open stratum and denote by $\bar{T}_{\alpha, i} \subset X^{\llbracket \alpha \rrbracket}$ the image of $\bar{S}_{\alpha, i}$.

Differing from the case of $Q / G r$, we shall work with the restriction of the obstruction sheaf to $\bar{T}_{\alpha, i}$. To this end, we let

$$
\overline{\mathcal{W}}_{\alpha, i}=\mathcal{O} b_{X}^{\llbracket \alpha \rrbracket} \otimes_{\mathcal{O}_{X} \llbracket \alpha \rrbracket} \mathcal{O}_{\bar{T}_{\alpha, i}} ;
$$

let $\bar{W}_{\alpha, i}$ be the associated vector bundle and let

$$
\bar{\xi}_{\alpha, i}:\left.\bar{E}_{\alpha}\right|_{\bar{T}_{\alpha, i}} \longrightarrow \bar{W}_{\alpha, i}
$$

be the surjective homomorphism induced by $\overline{\mathcal{E}}_{\alpha} \rightarrow \mathcal{O} b_{X}^{\llbracket \alpha \rrbracket}$.

We shall do the same for $(T X)_{0}^{\llbracket \alpha \rrbracket}$. We let $W_{\alpha, i}$ be the associated vector bundle of the restriction sheaf

$$
\tilde{\mathcal{W}}_{\alpha, i}=\mathcal{O} b_{T X}^{\llbracket \alpha \rrbracket} \otimes_{\mathcal{O}_{(T X)}}^{\llbracket \alpha \rrbracket} \mathcal{O}_{\widetilde{T}_{\alpha, i}},
$$

which is locally free over $\widetilde{T}_{\alpha, i}$; we let

$$
\tilde{\xi}_{\alpha, i}:\left.\widetilde{E}_{\alpha}\right|_{\widetilde{T}_{\alpha, i}} \longrightarrow \tilde{W}_{\alpha, i}
$$

be induced by $\widetilde{\mathcal{E}}_{\alpha} \rightarrow \mathcal{O} b_{T X}^{\llbracket \alpha \rrbracket}$.

Our next step is to show that possibly after re-indexing the $i$ 's we have

$$
\Psi_{\alpha}\left(\bar{T}_{\alpha, i} \cap U^{\llbracket \alpha \rrbracket}\right)=\widetilde{T}_{\alpha, i} \cap \mathcal{V}_{0}^{\llbracket \alpha \rrbracket} ;
$$

and that under the canonical isomorphism $\Psi_{\alpha}^{*} \bar{W}_{\alpha, i} \cong \tilde{W}_{\alpha, i}$ we have

$$
\Psi_{\alpha}\left(\tilde{\xi}_{\alpha, i}\left(\widetilde{D}_{\alpha, i}\right)\right)=\bar{\xi}_{\alpha, i}\left(\bar{D}_{\alpha, i}\right) \text { and } m_{\alpha, i}=\bar{m}_{\alpha, i} .
$$


The proof is straightforward. Let $x \in X$ be any element and let $\varphi_{x}: \mathcal{V}_{x} \rightarrow \mathcal{U}_{x} \subset X$ be the analytic open embedding provided by Lemma 2.4. By the construction of the projection $U \llbracket \alpha \rrbracket \rightarrow X$, its fiber over $x$ is canonically isomorphic to

$$
U^{\llbracket \alpha \rrbracket} \times_{X^{n}} \mathcal{U}_{X}^{n} \times_{T_{X} X} 0
$$

in which the map $\mathcal{U}_{x}^{n} \rightarrow T_{x} X$ is the composite

$$
\varpi_{x}: \mathcal{U}_{x}^{n} \stackrel{\cong}{\longrightarrow} \mathcal{V}_{x}^{n} \stackrel{\subset}{\longrightarrow} T_{x} X^{n} \stackrel{\text { ave }}{\longrightarrow} T_{x} X
$$

with the averaging map. Combining the local isomorphism Lemma (Lemma 2.2), the base change property of the obstruction sheaves (21), the invariance of the obstruction theory (Lemma 5.3) and the invariance of normal cone (22), we see immediately that to each stratum $S$ of $X^{\llbracket \alpha \rrbracket}$, the induced map

$$
S \times_{X^{n}}\left(\mathcal{U}_{x}\right)^{n} \stackrel{\varpi_{x}}{\longrightarrow} T_{X} X
$$

is a submersion. This shows that the standard stratification of $X^{\llbracket \alpha \rrbracket}$ induces the standard stratification of $\left(\mathcal{U}_{x}\right)^{\llbracket \alpha \rrbracket}{ }_{T_{X} X}$. By using $\mathcal{U}_{x} \cong \mathcal{V}_{x}$ and the open inclusion $\mathcal{V}_{x} \subset T_{x} X$, the standard stratification of $\left(T_{x} X\right)_{0}^{\llbracket \alpha \rrbracket}$ also induces the standard stratification of $\left(\mathcal{U}_{x}\right)^{\llbracket \alpha \rrbracket} \times_{T_{X} X} 0$. Therefore, the restrictions of the standard stratification of $\left(T_{X} X\right)_{0}^{\llbracket \alpha \rrbracket}$ and of the standard stratification of $X^{\llbracket \alpha \rrbracket}$ to $\mathcal{V}_{0}^{\llbracket \alpha \rrbracket} \times_{X} x$ coincide. Consequently, after re-indexing $\bar{C}_{\alpha, i}$, we will have (36).

Then by the base change property of the obstruction sheaves, we automatically have canonical isomorphism

$$
\Psi_{\alpha}^{*} \bar{W}_{\alpha, i} \cong \tilde{W}_{\alpha, i}
$$

applying the invariance results of the obstruction sheaf and of obstruction theory, we get the identity (37).

Once we have these, we immediately see that the section $\tilde{s}_{\alpha}$ induced by the isomorphism

$$
\left.\Psi_{\alpha}^{*}\left(\mathcal{O} b_{X}^{\llbracket \alpha \rrbracket}\right) \cong \mathcal{O} b_{T X}^{\llbracket \alpha \rrbracket}\right|_{\mathcal{V}_{0}^{\llbracket \alpha \rrbracket}}
$$

is a smooth section of $\mathcal{O} b_{X}^{\llbracket \alpha \rrbracket}$ over $U^{\llbracket \alpha \rrbracket}$; that it intersects transversally with the normal cone of $\mathcal{O} b_{X}^{\llbracket \alpha \rrbracket}$ and the collection $\left\{\widetilde{s}_{\alpha}\right\}$ satisfies the conclusions of Lemma 5.6 over $U \llbracket \alpha \rrbracket$.

To continue, we shall comment on the role of $0<c \ll 1$ and $0<\epsilon \ll 1$. In choosing sections $t_{\alpha}$ according to Lemma 5.6, we use the smallness of $c$ to force the resulting pseudo-cycle $\delta_{[n], Q}$ to lie entirely in the $2 c$-tubular neighborhood of the zero section of $Q_{0}^{n} / G r$. As to $\epsilon$, after picking $t_{\alpha}$ we use $\epsilon$ to choose pseudo-cycle representatives 
of $D\left(t_{\alpha}\right)$ to ensure that the resulting pseudo-cycle $\delta_{[n], Q}$ represents a homology class in $H_{*}(G r, \mathbb{Z})$.

After that, we pick a smooth embedding $g: X \rightarrow G r$ to pull the sections $t_{\alpha}$ back to sections $\tilde{t}_{\alpha}$ in $\mathcal{O} b_{T X}^{\llbracket \alpha \rrbracket}$. By choosing $g$ in general position, we can be sure that the degree of the discrepancy cycle $\delta_{[n], T X}$ constructed using $\widetilde{t}_{\alpha}$ coincides with the pull back of $\delta_{[n], Q}$ under the induced map $(T X)_{0}^{n} \rightarrow Q_{0}^{n}$. This time because each $D\left(\widetilde{t}_{\alpha}\right)$ is a zero-dimensional pseudo-cycle, they are cycles automatically. Likewise, because $\delta_{[n], Q}$ lies in the $2 c$-tubular neighborhood of the zero section of $Q_{0}^{n} / G r$, for $c$ sufficiently small, $\delta_{[n], T X}$ lies entirely in $\mathcal{V}_{0}^{n}$.

The next step is to form the sections $\widetilde{s}_{\alpha}$ of $\mathcal{O} b_{X}^{\llbracket \alpha \rrbracket}$ over $U^{\llbracket \alpha \rrbracket}$; restrict them to a compact subset $K^{\llbracket \alpha \rrbracket} \subset U^{\llbracket \alpha \rrbracket}$ and then extend the restrictions to all $X^{\llbracket \alpha \rrbracket}$ so that the resulting sections $\left\{s_{\alpha}\right\}$ satisfy the conclusion of Lemma 5.6. Now let $\Delta_{n} \subset X^{n}$ be the top diagonal $\{(x, \cdots, x) \mid x \in X\}$ as before and let $\Delta_{n, 3 c}$ be the $3 c$-tubular neighborhood of $\Delta_{n}$ in $X^{n}$. Because $c$ is sufficiently small, we can choose a compact $K \subset X^{n}$ so that

$$
\Delta_{n, 3 c} \subset K \subset \mathcal{V}_{0}^{n} \subset X^{n} .
$$

Thus if we choose $K^{\llbracket \alpha \rrbracket}=X^{\llbracket \alpha \rrbracket} \times_{X^{n}} K$, then the discrepancy cycle $\delta_{[n]}$ constructed using $D\left(s_{\alpha}\right)$ is entirely contained in $\Delta_{n, 3 c}$; thus is contained in $U \llbracket \alpha \rrbracket$; thus coincide with $\Psi_{[n]}\left(\delta_{[n], T X}\right)$. Here the last statement holds because $\delta_{[n], T X} \subset \mathcal{V}_{0}^{n}$.

This completes the proof of Lemma 6.1.

\section{The case of complex manifolds}

To generalize this theorem to cover all compact, smooth three-dimensional complex manifolds, we first need to define their Hilbert schemes of subschemes and their Donalson-Thomas invariants. The technique developed in this work readily covers the case of zero-dimensional invariants.

We now construct the Hilbert scheme of points for such complex manifolds $X$. We shall achieve this goal indirectly by quoting the invariance results proved in this paper. We first cover $X$ by open subsets $U_{\alpha}$ such that each is realized as an open subset of $\mathbf{A}^{3}$. By viewing $U_{\alpha}$ as an open subset of $\mathbf{A}^{3}$, we define the Hilbert scheme of $n$-points

$$
I_{U_{\alpha}}(0, n) \subset I_{\mathbf{A}^{3}}(0, n)
$$

be the open (analytic) subscheme of all $\xi \in I_{\mathbf{A}^{3}}(0, n)$ whose supports lie in $U_{\alpha}$. For any pair $U_{\alpha}$ and $U_{\beta}$, the Lemma 2.2 ensures that the open subscheme

$$
I_{U_{\alpha \beta}}(0, n) \subset I_{U_{\alpha}}(0, n)
$$


is canonically isomorphic to the open subscheme

$$
I_{U_{\alpha \beta}}(0, n) \subset I_{U_{\beta}}(0, n) .
$$

Thus the collection $I_{U_{\alpha}}(0, n)$ glue to form an analytic scheme, the Hilbert scheme of $n$ points in $X$ :

$$
I_{X}(0, n)
$$

The scheme $I_{X}(0, n)$ comes with the usual obstruction sheaf, obstruction theory and virtual normal cone. Because of the invariance results stated or proved in this paper, the obstruction sheaves $\mathcal{O} b_{\alpha}$ of $I_{U_{\alpha}}(0, n)$ glue together to form the obstruction sheaf of $I_{X}(0, n)$. It can also be defined as the traceless relative extension sheaf of the universal ideal sheaf of $\mathcal{Z} \subset X \times I_{X}(0, n)$ :

$$
\mathcal{O} b=\mathcal{E} x t_{\pi_{2}}^{2}\left(\mathcal{I}_{\mathcal{Z}}, \mathcal{I}_{\mathcal{Z}}\right)_{0} .
$$

Over each open $I_{U_{\alpha}}(0, n)$, we can find locally free sheaf $\mathcal{E}_{\alpha}$ making the obstruction sheaf $\mathcal{O} b_{\alpha}$ of $I_{U_{\alpha}}(0, n)$ its quotient sheaf; we can also construct its associated normal cone $C_{\alpha}$ in the associated vector bundle $E_{\alpha}$. Using

$$
C_{\alpha} \subset E_{\alpha} \text { and } \mathcal{E}_{\alpha} \longrightarrow \mathcal{O} b_{\alpha},
$$

we can define the notion of smooth sections of $\mathcal{O} b_{\alpha}$ and when smooth section $s_{\alpha}$ of $\mathcal{O} b_{\alpha}$ intersects transversally with the normal come. Because such notion is consistent when restricted to open subsets $I_{U_{\alpha \beta}}(0, n)$, we can make sense of smooth sections of $\mathcal{O} b$ on $I_{X}(0, n)$ and when it intersects transversally with the normal cone of $\mathcal{O} b$.

We then define the virtual cycle $I_{X}(0, n)$ be the homology class

$$
[D(s)] \in H_{0}\left(I_{X}(0, n) ; \mathbb{Z}\right)
$$

represented by the pseudo-cycle $D(s)$ constructed by intersecting the graph of $s$ with the normal cone in $\mathcal{O} b$.

To show that the cycle $[D(s)]$ is well-defined, namely it is independent of the choice of $s$, we need to show that for different smooth sections $s$ the cycles $D(s)$ are homotopy equivalent. This is true because we can find a stratification of $I_{X}(0, n)$ and of the cone so that each stratum is the complement of finitely many closed analytic subvarieties in a closed analytic variety.

Combined, this proves

Theorem 7.1 Let $X$ be a compact, smooth three dimensional complex manifold. Then the so constructed Hilbert scheme of points $I_{X}(0, n)$ has a well-defined virtual cycle

$$
\left[I_{X}(0, n)\right]^{\mathrm{vir}} \in H_{0}\left(I_{X}(0, n) ; \mathbb{Z}\right)
$$


that is represented by the cycle $D(s)$ after intersecting a smooth section $s$ transversally with the normal cone in the obstruction sheaf $\mathcal{O} b$.

Once the virtual cycle is constructed, then the proof of this paper applies line to line to $I_{X}(0, n)$ to conclude that

$$
\operatorname{deg}\left[I_{X}(0, n)\right]^{\mathrm{vir}}
$$

is expressible by the same universal expression in its Chern numbers as other projective threefolds. Thus

\section{Theorem 7.2 The identity}

$$
\sum_{n} \operatorname{deg}\left[I_{X}(0, n)\right]^{\mathrm{vir}} q^{n}=M(-q)^{c_{3}\left(T_{X} \otimes K_{X}\right)}
$$

holds for all compact, smooth three dimensional complex manifolds.

The Hilbert scheme of ideal sheaves of curves for any complex manifold $X$ can also be defined. In case $X$ has dimension three, one can also define its virtual cycle and its Donaldson-Thomas series, along the lines of the work [8].

Acknowledgement The authors is partially supported by NSF grants DMS-0200477 and DMS-0244550.

\section{References}

[1] IV Artamkin, On the deformation of sheaves, Izv. Akad. Nauk SSSR Ser. Mat. 52 (1988) 660-665, 672 (Russian) MR954302 English translation: Math. USSR. Izvestiya, 32 (1989) 663-668

[2] K Behrend, Donaldson-Thomas invariants via microlocal geometry arXiv: math. AG/0507523

[3] K Behrend, B Fantechi, Symmetric obstruction theories and Hilbert schemes of points on threefolds arXiv:math.AG/0512556

[4] K Behrend, B Fantechi, The intrinsic normal cone, Invent. Math. 128 (1997) 45-88 MR1437495

[5] S K Donaldson, R P Thomas, Gauge theory in higher dimensions, from: "The geometric universe science, geometry, and the work of Roger Penrose (Oxford, 1996)", (S S Huggett, et al, editors), Oxford Univ. Press (1998) 31-47 MR1634503

[6] M Levine, $\mathbf{R}$ Pandharipande, Algebraic Cobordism revisited arXiv: math. AG/0605196 
[7] J Li, G Tian, Virtual moduli cycles and Gromov-Witten invariants of algebraic varieties, J. Amer. Math. Soc. 11 (1998) 119-174 MR1467172

[8] J Li, G Tian, Comparison of algebraic and symplectic Gromov-Witten invariants, Asian J. Math. 3 (1999) 689-728 MR1793677

[9] M Maruyama, Moduli of stable sheaves II, J. Math. Kyoto Univ. 18 (1978) 557-614 MR509499

[10] D Maulik, N Nekrasov, A Okounkov, R Pandharipande, Gromov-Witten theory and Donaldson-Thomas theory I arXiv:math.AG/0312059

[11] D Maulik, N Nekrasov, A Okounkov, R Pandharipande, Gromov-Witten theory and Donaldson-Thomas theory II arXiv:math.AG/0406092

[12] S Mukai, Symplectic structure of the moduli space of sheaves on an abelian or K3 surface, Invent. Math. 77 (1984) 101-116 MR751133

[13] R P Thomas, A holomorphic Casson invariant for Calabi-Yau 3-folds, and bundles on K3 fibrations, J. Differential Geom. 54 (2000) 367-438 MR1818182

Department of Mathematics, Stanford University

Stanford, CA 94305, USA

jli@math.stanford.edu

Proposed: Jim Bryan

Seconded: Lothar Goettsche, Eleny Ionel

Received: 27 April 2006 Accepted: 10 October 2006 